\title{
Random Records and Cuttings in Binary Search Trees
}

\author{
Cecilia Holmgren
}

U.U.D.M. Report 2007:52

ISSN 1101-3591

Filosofie Licentiatavhandling

i matematik

som framläggs för offentlig granskning

den 31 oktober, kl 10.15, sal 64119

på Ångströmlaboratoriet i Uppsala

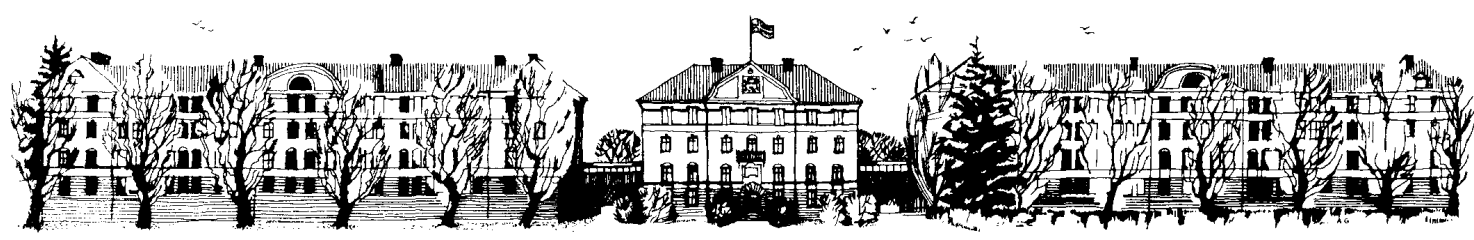

Department of Mathematics

Uppsala University 



\title{
Random Records and Cuttings in Binary Search Trees
}

\author{
Cecilia Holmgren
}

October 11, 2007 



\section{Contents}

I 2

1 Introduction . . . . . . . . . . . . . . . 3

1.1 Random Graphs . . . . . . . . . . . . . . . . 3

1.2 Random Trees . . . . . . . . . . . . . . . . . . . . 3

1.3 Random Records and Cuttings . . . . . . . . . . 9 9

1.4 Random Records and Cuttings in Galton-Watson Trees 10

2 Aims . . . . . . . . . . . . . . . . . . . . 12

3 Results and Discussion . . . . . . . . . . . . . . . . 12

3.1 "Random Records and Cuttings in Binary Search Trees" and Comparisons with Janson's Findings for the Complete Binary Tree . . . . . . . . . . . . . . . . . 12

3.2 Ideas and Preliminary Results on Random Records and Cuttings in Split Trees . . . . . . . . . . . . 18

4 Conclusions . . . . . . . . . . . . . . . . . 22

5 Acknowledgements . . . . . . . . . . . . . . 23

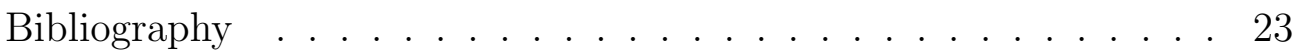

II Manuscript, to be Submitted 26

"Random Records and Cuttings in Binary Search Trees"

(Referred to as The Paper in Part I) . . . . . . . . 27 
Part I 


\section{Introduction}

\subsection{Random Graphs}

In 1947 the famous Hungarian mathematician Paul Erdös introduced the notion of a random graph in one of his papers. It was used to prove the existence of a graph with a specific Ramsey property. Many subsequent workers have also found probability theory to be very useful for proving existence. The model invented by Erdős is natural and is defined as follows: Choose a graph at random with equal probabilities from the set of all graphs of vertex set $\{1,2, \ldots, n\}$. Consequently, there are obviously $2^{\left(\begin{array}{l}n \\ 2\end{array}\right)}$ graphs since one decides for each pair of vertices if the edge that connects the pair is absent or present. Equivalently this can be described as the probability space $(\Omega, \mathscr{F}, \mathscr{P})$, where $\Omega$ is the set of all graphs with vertex set $\{1,2, \ldots n\}$,

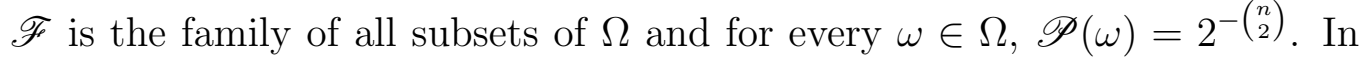
simple words this means that independently $\left(\begin{array}{l}n \\ 2\end{array}\right)$ tosses of a coin are done where "turning up heads" means "drawing an edge". See e.g [2] and [16].

The theory of random graphs was built up a decade later from 19591968 by a series of articles published by Paul Erdös and his colleague Alfred Rényi, where they use probabilistic methods to solve different problems in graph theory. In the late seventies Béla Bollobás became the leading scientist in the field with dozens of articles, which became the framework of his book "Random graphs" [2] published in 1985. In 2000 the second book "Random Graphs" [16] was published by Svante Janson, Tomasz Łuczak and Andrzej Rucínski, providing a summary of developments achieved by them and other mathematicians in the field since 1985.

The theory today has developed into a branch of discrete mathematics located at the intersection of graph theory, combinatorics and probability theory, with applications to for example theoretical computer science, bioinformatics and reliability of transportation and communication networks. In general a random graph is a graph constructed by a random procedure. Usually one does not distinguish between different random graphs with the same distribution. Typically one lets the sizes of the graphs tend towards infinity, thus the theory of random graphs is by the nature of its design often asymptotic. See [16].

\subsection{Random Trees}

Random trees are a special class of random graphs.

A rooted tree is a connected graph with one vertex defined as the root, and where there is a unique path of edges from each vertex to the root and 
where there are no cycles in the graph.

The depth of a vertex $v$ in the tree is its distance to the root, that is the number of edges in the unique path from the vertex $v$ to the root of the tree. The height of the tree is the maximal depth in the tree. The vertices are also called nodes.

A random tree is a tree that is generated from some probability procedure that describes how the edges or vertices are distributed. Of course there are many different kinds of random trees. In this thesis according to the title the "binary search tree" is the one that is mostly studied. This tree belongs to the class of trees of logarithmic height. These are trees where the height for large $n$ is approximately $C \log n$, where $C$ is a constant. This is an important class and includes many different kinds of trees of which several are used to describe algorithms in Computer Science. The binary search tree also belongs to a subclass of the trees of logarithmic height that is the "split" trees. The project that I am currently working on is to extend the methods used for the binary search tree in The Paper to work for general split trees.

The most wellknown tree of logarithmic height is the fixed "complete binary" tree of $n$ vertices. A binary tree is a tree where each vertex in the tree have between 0 and 2 children. Typically the child vertices are called the "left" and "right" child respectively. The complete binary tree of size $n$ is a binary tree where each vertex have exactly 2 children, except for those that are at the level of the height (the maximal depth) of the tree that of course have no children and possibly those that are in one level above. The vertices in this level have to chair the remaining vertices as their children so that the sum of all vertices in the tree is $n$. Also at the height of the tree (if this level is not completely filled) all vertices must be as far left as possible. More precisely, the complete binary tree is a tree of height $m=\left\lfloor\log _{2} n\right\rfloor$, with $2^{k}$ vertices of height $k, 0 \leq k<m$ and $n-2^{m}+1$ vertices of height $m$, where the vertices of height $m$ have the leftmost positions among the $2^{m}$ possible ones. See e.g. [18, p.401]. Figure 1 illustrates a complete binary tree.

The binary search tree that one frequently uses to sort data is almost as old as Computer Science. Donald Knuth [19] gives a detailed account of the research that has been done on these trees, where he himself has contributed a lot. For the binary search tree there are many equivalent ways to describe the tree for which several are used in The Paper. The following description of a binary search tree is the standard one (although there are other descriptions that are usually more useful in applications) and describes how to generate a binary search tree as a random graph model: Draw a number which we call a key uniformly and independently from a set $\{1,2 \ldots n\}$, and associate the key to the root. Draw another key independently from the remaining 


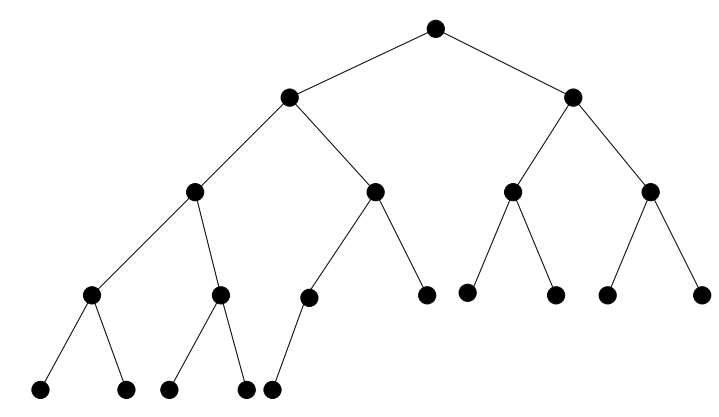

Figure 1: A picture of a complete binary tree, where the root is at the top of the tree. In this tree there is 1 root vertex at depth zero. There are 2 vertices at depth one, 4 vertices at depth two, 8 vertices at depth three and 5 vertices are at the height of the tree which is four.

numbers and place it at the left child of the root if it is smaller than the root's key and place it to the right if it is larger. Then one proceeds growing the tree incrementally: when the $k$ 'th key is to be placed one starts at the root and recursively find the subtree to which the key is going to belong to, by comparing the key to the current root's key and go to the right if it is larger and to the left otherwise. Eventually one locates an empty vertex (subtree) and then one places the key at this position. When all keys are placed one gets a binary tree on $n$ vertices. See e.g. [6] and [7]. Figure 2 illustrates an example of a binary search tree generated by keys as described above.

A binary tree of size $n$ has at least height $\lfloor\lg n\rfloor:=\left\lfloor\log _{2} n\right\rfloor$ (as for example the complete binary tree) and at most height $n$ (if all vertices have just one child). The height of the binary search tree is as mentioned above shown to be logarithmic and has been extensively studied, see [23], [4] and [8]. Another behaviour of the tree that has been studied a lot is the depth for which most vertices are in the tree (equivalently for a binary search tree of size $n$ this means the depth $D_{n}$ for the last vertex to be inserted), see e.g [19] and [20]. Also related information of the tree structure has been studied such as the profile (that is the number of vertices at a given height $k$ ), see [3].

Quicksort is the algorithm version of a random binary search tree. This is the standard sorting algorithm in Unix systems used to sort an array of $n$ distinct numbers, see e.g. [13]. Quicksort is described in Subsection 1.2.2 in The Paper below.

As mentioned above the binary search tree belongs to the class of trees that are called random "split trees" introduced by Devroye. The definition 


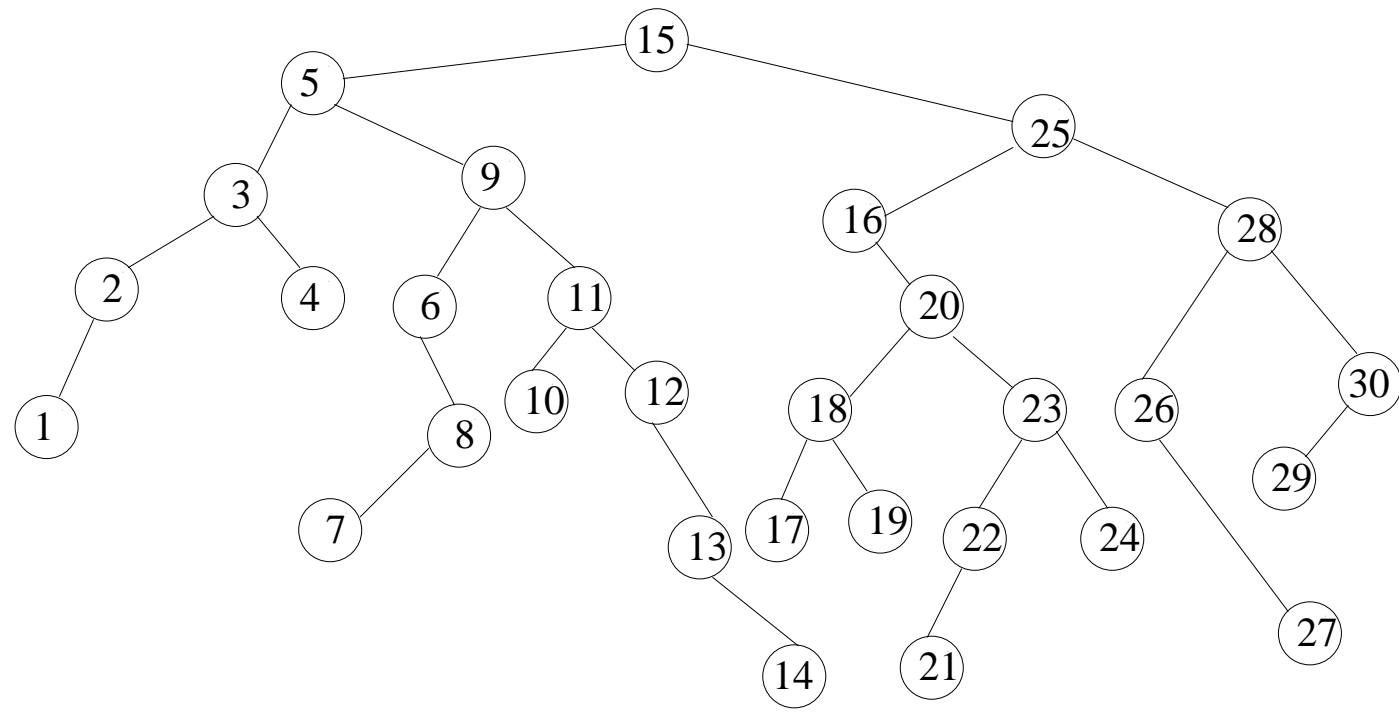

Figure 2: A picture of a binary search tree generated by the "key sequence" 15, 5, 3, 25, 9, 6, 28, 16, 11, 20, 2, 1, 18, 23, 30, 26, 17, 19, 12, 10, 4, 13, 27, 8, 22, 14, 24, 7, 29, 21. The key 15 is in the root at depth zero. The keys 5 and 25 are at depth one; 3, 9, 16 and 28 are at depth two; 2, 4, 6, 11, 20, 26 and 30 are at depth three; 1, 8, 10, 12, 18, 23, 27 and 29 are at depth four; 7, 13, 17, 19, 22 and 24 are at depth five; and finally 14 and 21 are at the maximal depth which is six in this case. Note that if the keys associated to the vertices in this figure are deleted they can be recovered, since this labelling of the vertices is the only labelling that is possible from the set of keys $\{1,2,3 \ldots, 30\}$.

of random split trees and important results for them are found in [5]. Below the definition of a random split tree is given:

A skeleton tree $T_{b}$ of branch factor $b$ is an infinite rooted tree in which each vertex has exactly $b$ children that are numbered $1,2, \ldots, b$. A split tree is a skeleton tree of branch factor $b$ in which $n$ balls are given to a collection of vertices and where each vertex can hold up to $s$ balls. Let $N(v)$ denote the number of balls given to the vertices in the subtree rooted at vertex $v$ and $C(v)$ be the number of balls that are given to vertex $v$. A vertex $v$ is a leaf if $C(v)=N(v)>0$; this means there are not any balls linked to the children of $v$. A vertex $v$ is useless if $N(v)=0$ why such a vertex is eliminated from the tree. However, $C(v)=0$ is allowed. The random split tree with parameters $b, s, s_{0}, s_{1}, \mathcal{V}$ and $n$ can now be introduced. The parameters $b, s$ and $n$ are as for split trees. The additional integers $s_{0}$ and $s_{1}$ are needed to describe how 
the balls are distributed and have to satisfy the inequality

$$
0 \leq s_{0} \leq s, 0 \leq b s_{1} \leq s+1-s_{0}
$$

In the "split tree generating algorithm" that is presented below, one sees why this inequality is relevant. The last parameter $\mathcal{V}$ is the so called random "splitting vector" $\left(V_{1}, V_{2} \ldots V_{b}\right)$ of probabilities, where $\sum_{i} V_{i}=1, V_{i} \geq 0$. A random split tree is a skeleton tree $T_{b}$ in which each vertex $v$ is given an independent copy of $\mathcal{V}=\left(V_{1}, V_{2} \ldots V_{b}\right)$. One also assumes that the components of $\left(V_{1}, V_{2} \ldots V_{b}\right)$ are identically distributed. (If they are not one achieves this goal with a random permutation anyway.) This gives (because of $\sum_{i} V_{i}=1$ ) that $\mathbf{E}\{V\}=\frac{1}{b}$.

Below the algorithm to describe how the $n$ balls are distributed over the vertices is given. Initially there are no balls, that is $C(v)=0$ for each vertex $v$. Adding a ball to a tree rooted at $v$ is done by the following procedure.

\section{The split tree generating algorithm:}

1. If $v$ is not a leaf choose child $i$ with probability $V_{i}$. This increases $N(v)$ by 1 . Recursively add the ball to the subtree rooted at child $i$, by the rules given in steps 1,2 and 3 .

2. If $v$ is a leaf and $C(v)=N(v)<s,(s$ is the capacity of the vertex) then add the ball to $v$ and stop. Thus, $C(v)$ and $N(v)$ increase by 1 .

3. If $v$ is a leaf and $C(v)=N(v)=s$ the ball cannot be placed at $v$ since it is occupied by the maximum number of balls it can hold. Then let $N(V)=s+1$ and $C(v)=s_{0}$, by placing $s_{0} \leq s$ randomly chosen balls at $v$ and $s+1-s_{0}$ balls to its children. Do this by first giving $s_{1}$ randomly chosen balls to each of the $b$ children. The remaining $s+1-s_{0}-b s_{1}$ balls are placed by choosing a child for each ball independently according to the probability vector $\mathcal{V}=\left(V_{1}, V_{2} \ldots V_{b}\right)$. Then use the algorithm described in steps 1, 2 and 3 to the tree rooted at the selected child.

Note that if $s_{0}>0$ the procedure does not need to be repeated since no child could reach the capacity $s$, whereas in the case $s_{0}=0$ the procedure may have to be repeated several times. Also note that every nonleaf vertex has $C(v)=s_{0}$ balls and every leaf has $0<C(v) \leq s$ balls. See figure 3 , where two examples of split trees are illustrated.

In a binary search tree the splitting vector $\left(V_{1}, V_{2}\right)$ is distributed as $(U, 1-U)$, where $U$ is uniform $[0,1]$ or equivalently $U$ is beta $(1,1)$. In fact 


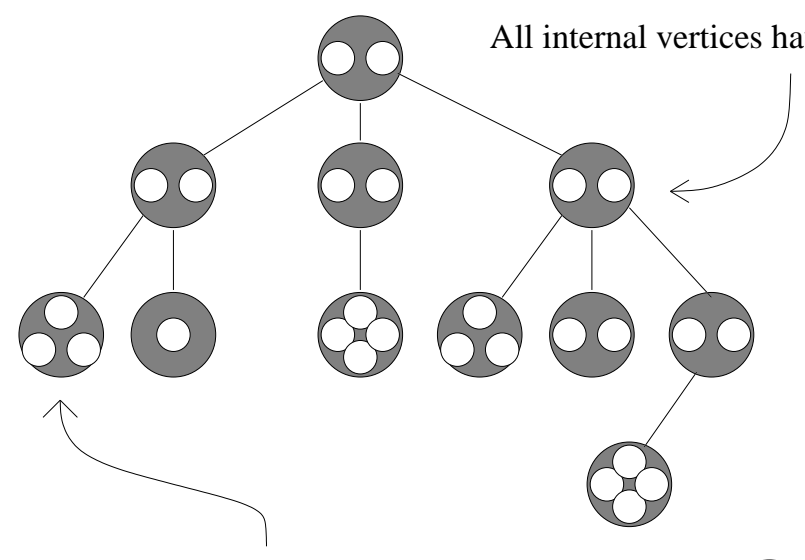

All leaf vertices have between 1 and $s=4$ balls.

Note that s_ $_{1}=0$.
All internal vertices have s_ $0=0$ balls.

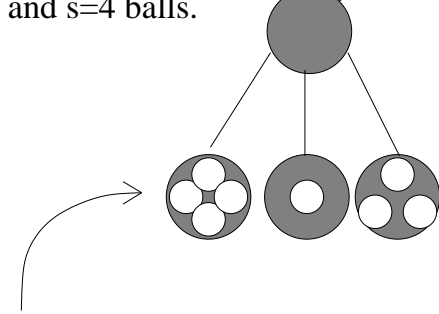

All leaf vertices have between 1 and $s=4$ balls.

Note that $s_{-} 1=1$.

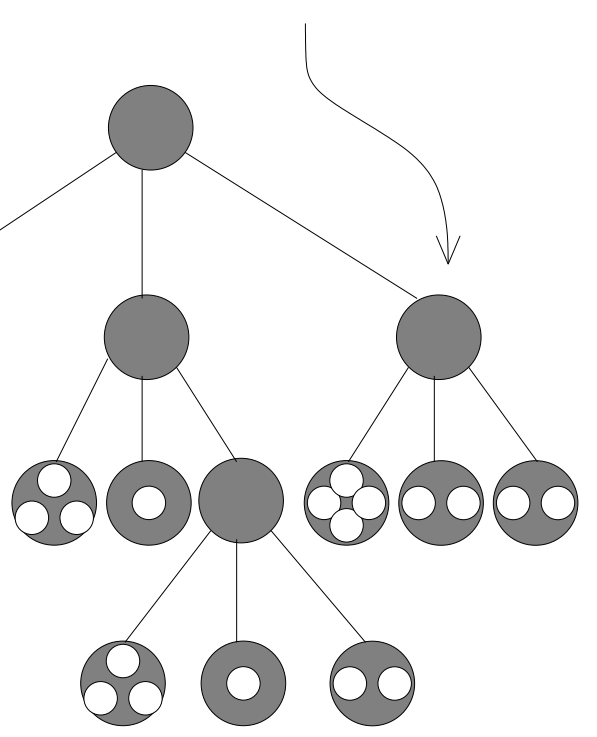

Figure 3: This picture illustrates two split trees. The left one has (split tree) parameters $b=3, s=4, s_{0}=2$ and $s_{1}=0$, whereas the right one has parameters $b=3, s=4, s_{0}=0$ and $s_{1}=1$. Note that there is in general no simple relationship between the number of vertices and the number of balls.

for many important split trees the components in the split vector $\mathcal{V}$ are betadistributed. The other parameters for the binary search tree considered as a split tree are $s=1, s_{0}=1, s_{1}=0$ and $b=2$.

In a split tree where the number of balls $n>s$ there are $s_{0}$ balls in the root vertex and the cardinalities of the $b$ subtrees are distributed as $\left(s_{1}, \ldots, s_{1}\right)$ plus a multinomial vector $\left(n-s_{0}-b s_{1}, V_{1}, \ldots V_{b}\right)$. Thus, conditioning on the random $\mathcal{V}$-vector that belongs to the root the subtrees rooted at the children have cardinalities close to $n V_{1}, \ldots n V_{b}$. This is often used in applications of random binary search trees. In particular in The Paper below I use this to estimate the subtree sizes. However, $n$ is there described as the number of vertices instead of balls, but they are the same number in a binary search tree which is not true for split trees in general.

In [5] Devroye presents a strong law and a central limit law for the depth $D_{n}$ of the last vertex in a random split tree of $n$ vertices and split vector $\mathcal{V}$. 
(As for the binary search, for a split tree in general this gives the depth $D_{n}$ of which most vertices are close to.) This is closely related to the profile of the tree. Devroye defines

$$
\begin{aligned}
\mu & :=b \mathbf{E}\left\{V \ln \frac{1}{V}\right\}, \\
\sigma^{2} & :=b \mathbf{E}\left\{V \ln ^{2} V\right\}-\mu^{2},
\end{aligned}
$$

and the result he gets is

Theorem 1.1. Let $D_{n}$ be the depth of the last vertex in a random split tree with $n$ vertices and split vector $\mathcal{V}$. If $\mu \neq 0$ and $\mathbf{P}\{V=1\}=0$, then

$$
\frac{D_{n}}{\ln n} \rightarrow \frac{1}{\mu}
$$

and $\frac{\mathbf{E}\left\{D_{n}\right\}}{\ln n}$ tends to the same limit. Furthermore, if $\sigma>0$, then

$$
\frac{D_{n}-(\ln n) / \mu}{\sqrt{\sigma^{2}(\ln n) / \mu^{3}}} \stackrel{d}{\rightarrow} N(0,1),
$$

where $N(0,1)$ denotes the standard Normal distribution and $\stackrel{d}{\rightarrow}$ denotes convergence in distribution.

Another important class of trees that is not studied in my thesis are the conditioned "Galton-Watson" trees. Trees that are of this type are for example ordered (plane) trees, Cayley trees and some binary trees that are not of logarithmic height. The shape of the Galton-Watson trees are very different from the trees of logarithmic height. Thus, the result of the random record distribution which is presented in The Paper differs completely from the corresponding result which Janson gets in [15] for these types of trees. Also the methods used in the proofs are totally different. The Galton-Watson trees are obtained as the family tree of a Galton-Watson process conditioned on a given total size. One lets $\xi$ be a non negative integer random variable and considers the Galton Watson process with offspring distribution $\xi$, where one assumes $\mathbf{E}(\xi)=1$ and $0<\operatorname{Var}(\xi)<\infty$. This means that $\xi$ is the parameter (depending on its distribution) defining which type of subclass the Galton-Watson tree belongs to.

\subsection{Random Records and Cuttings}

"Cutting down" trees was first studied by Meir and Moon in 1970, see [21]. The trees they considered were Cayley trees. The process of "Cutting down" a tree can be described as follows: 
Let $T$ be a rooted tree. Make one cut by choosing a vertex at random. Delete this vertex so that the tree separates into two parts and keep only the part containing the root. Continue recursively until only the root is left. Let $X(T):=X_{v}(T)$ be the random number of cuts to destroy the tree. Alternatively one can consider edges and the procedure is obviously described in the same way with $X_{e}(T)$ as the corresponding random variable.

For other papers of "Cutting down" trees see the papers [22], [9], and $[12]$.

In [15] Janson introduces a random variable that is equally distributed as the cutting down random variable and is better suited to understand the underlying probability theory of the process. He considers records of a tree defined as follows: Let each vertex have a random variable $\lambda_{v}$ attached to it and assume that these random variables are independent identically distributed, where the distribution is continuous. Then say $\lambda_{v}$ is a record if it is the smallest value in the path from the root to $v$.

Janson concludes that the number of records given is also $X_{v}(T)$. (As for the cuts one can obviously consider the edge version instead.) To see why one gets the same variable $X_{v}(T)$ for these two models one can use a natural coupling argument explained as follows: First generate the values $\lambda_{v}$ and then cut the tree each time choosing the vertex with the smallest $\lambda_{v}$ of the remaining ones. This gives the cutting down procedure and a vertex $v$ is cut at some time if and only if the attached $\lambda_{v}$ value is a record. Hence, the number of records equals (in distribution) the number of cuts. In The Paper the record version is considered. To avoid misunderstanding note that the values attached to the nodes that are denoted by $\lambda_{v}$ 's have nothing to do with the values that are called keys (see Section 1.2) which are used to generate the binary search tree.

\subsection{Random Records and Cuttings in Galton-Watson Trees}

In [15] Janson uses the record model just described to find the asymptotic distribution of the normalized $X\left(T_{n}\right)$ ( $T_{n}$ denotes a tree of $n$ nodes) for random trees that can be considered as conditioned Galton-Watson trees. These are defined in Subsection 1.2 above. Since both the records and the tree are random $X\left(T_{n}\right)$ is considered in two ways. The first is to regard $X\left(T_{n}\right)$ as a random variable, obtained by picking a random tree $T_{n}$ and then a random cutting of it. Thus, the limit result after normalization in $[15$, Theorem 1.6] is stated as:

Theorem 1.2. Let $T_{n}$ be a conditioned Galton-Watson tree of size $n$ defined 
by the offspring distribution $\xi$. Then

$$
\frac{X\left(T_{n}\right)}{(\operatorname{Var}(\xi) n)^{\frac{1}{2}}} \stackrel{d}{\longrightarrow} Z,
$$

where $Z$ has the Rayleigh distribution with density $x e^{\frac{-x^{2}}{2}}, x>0$. Moreover, if $\mathbf{E}\left(\xi^{m}\right)<\infty$ for all $m>0$, then all moments converge in (5) and thus for every $r>0$,

$$
\mathbf{E}\left(X\left(T_{n}\right)^{r}\right) \sim(\operatorname{Var}(\xi) n)^{\frac{r}{2}} \mathbf{E}\left(Z^{r}\right)=2^{\frac{r}{2}} \Gamma\left(\frac{r}{2}+1\right)(\operatorname{Var}(\xi) n)^{\frac{r}{2}} .
$$

The other point of view is to study $X\left(T_{n}\right)$ as a random variable conditioned on the tree $T_{n}$. This means that one considers the random procedure in two steps. First one picks a random tree $T=T_{n}$ that one keeps fixed and then one considers random cuttings of it. This gives a random variable $X\left(T_{n}\right)$ with a distribution that depends on $T$. Define $\mu_{T_{n}}$ as the random probability distribution of $\frac{X\left(T_{n}\right)}{(\operatorname{Var}(\xi) n)^{\frac{1}{2}}}$ given the tree $T_{n}$. Let $B_{e x}$ denote the normalized Brownian excursion (this is a random function), see e.g [1]. Then there exists for every constant $c>0$ a random probability measure $\nu_{c B_{e x}}$, see [15]. Using this point of view part of the other main result [15, Theorem 1.10] is stated as:

Theorem 1.3. Let $T_{n}$ be a conditioned Galton-Watson tree of size $n$, then

$$
\mu_{T_{n}} \stackrel{d}{\longrightarrow} \nu_{2 B_{e x}} \text {. }
$$

Moreover, moment convergence holds, that is for every $k \geq 1$

$$
\frac{\mathbf{E}\left(X\left(T_{n}\right)^{k} \mid T_{n}\right)}{(\operatorname{Var}(\xi) n)^{\frac{k}{2}}} \stackrel{d}{\longrightarrow} \int x^{k} d \nu_{2 B_{e x}}(x) .
$$

The main idea of the proofs Janson presents to show the results in [15] is to use the method of moments, see [16, Section 6.1]. This method is one of the oldest to prove convergence in distribution. The moments of a random variable $X$ are the numbers $\mathbf{E}\left(X^{k}\right), k \geq 1$. One says that a distribution is determined by its moments if $X$ has finite moments and every random variable with the same moments as $X$ has the same distribution. This is true for example the normal and Poisson distribution. The standard version is stated as:

Theorem 1.4. Let $Z$ be a random variable with a distribution that is determined by its moments. If $X_{1}, X_{2}, \ldots$ are random variables with finite moments such that $\mathbf{E}\left(X_{n}^{k}\right) \rightarrow \mathbf{E}\left(Z^{k}\right)$ as $n \rightarrow \infty$ for every integer $k \geq 1$, then $X_{n} \stackrel{d}{\rightarrow} Z$.

For a proof of this see e.g. [11, Theorem 5.8.6]. 


\section{Aims}

In [14] Janson finds the asymptotic distribution after normalization of the record random variable $X\left(T_{n}\right)$ for the complete binary tree. (Recall the definition of the complete binary tree in Subsection 1.2 above and the definition of $\left(X\left(T_{n}\right)\right.$ in Subsection 1.3.) The method he uses is to approximate $X\left(T_{n}\right)$ by a sum of independent random variables derived from $\left\{\lambda_{v}\right\}$, defined in Subsection 1.3. The aim of the present study is to determine whether Janson's method could be extended to find the asymptotic distribution of the normalized $X\left(T_{n}\right)$ for the binary search tree, defined in Subsection 1.2 above. Both of these trees belong to the class of trees of logarithmic height thus many properties are similar for these trees; but in contrast to the complete binary tree that is deterministic the binary search tree is a random tree and therefore substantially more complex.

\section{Results and Discussion}

\section{1 "Random Records and Cuttings in Binary Search Trees" and Comparisons with Janson's Findings for the Complete Binary Tree}

Below we use the usual notations $\lg$ for the 2-logarithm and $\ln$ for the $e$ logarithm, and let $\{x\}=x-\lfloor x\rfloor$ denote the fractional part of a real number $x$.

Then the result of $X\left(T_{n}\right)$ for the complete binary tree, see [14, Theorem 1.1], is stated as:

Theorem 3.1. Considering subsequences and suppose that $n \rightarrow \infty$ such that $\{\lg n-\lg \lg n\} \rightarrow \gamma \in[0,1]$. Then

$$
\frac{\left(X_{v}\left(T_{n}\right)-\frac{n}{\lg n}-\frac{n \lg \lg n}{\lg ^{2} n}\right)}{\frac{n}{\lg ^{2} n}} \stackrel{d}{\rightarrow}-W_{\gamma},
$$

where $W_{\gamma}$ has an infinitely divisible distribution with characteristic function

$$
\mathbf{E} e^{i t W}=\exp \left(i f(\gamma) t+\int_{0}^{\infty}\left(e^{i t x}-1-i t x \mathbf{1}[x<1]\right) d \nu_{\gamma}(x)\right),
$$

where the Lévy measure $\nu_{\gamma}$ is supported on $(0, \infty)$ and has density

$$
\frac{d \nu_{\gamma}}{d x}=\frac{2^{\{\lg x+\gamma\}}}{x^{2}} .
$$


The same result holds for $X_{e}\left(T_{n}\right)$.

The Lévy measure $c x^{-2}$ gives a weakly 1-stable distribution. That a distribution of a random variable $Z$ is $\alpha$-stable for $\alpha \in(0,2]$ means that a sequence of identically distributed random variables $Z_{k}, k \geq 1$ (such that for all $k, Z_{k} \stackrel{d}{=} Z$ ), satisfy for all $n, \sum_{k=1}^{n} Z_{k} \stackrel{d}{=} c_{n} Z+d_{n}$, where $c_{n}=n^{\frac{1}{\alpha}}$. The distribution is strictly stable if for all $n, d_{n}=0$ and weakly stable otherwise. See [10, Section XVII.3 ]. The measure $\nu_{\gamma}$ is similar to this but with periodic fluctuations, so the distribution of $W_{\gamma}$ is roughly similar to a 1-stable distribution.

The result of $X\left(T_{n}\right)$ I find for the binary search tree in Theorem $1.1 \mathrm{in}$ The Paper below is similar to Theorem 3.1 and can be stated as

Theorem 3.2. Suppose that $n \rightarrow \infty$. Then,

$$
\frac{\left(X_{v}\left(T_{n}\right)-\frac{n}{2 \ln n}-\frac{n \ln \ln n}{2 \ln ^{2} n}\right)}{\frac{n}{4 \ln ^{2} n}} \stackrel{d}{\rightarrow}-W,
$$

where $W$ has an infinitely divisible distribution with characteristic function

$$
\mathbf{E} e^{i t W}=\exp \left(i(-3-2 \ln 2+2 \gamma) t+\int_{0}^{\infty}\left(e^{i t x}-1-i t x \mathbf{1}[x<1]\right) d \nu(x)\right),
$$

where $(\gamma=0.577 \ldots$ is the Euler constant) and the Lévy measure $\nu$ is supported on $(0, \infty)$ and has density

$$
\frac{d v}{d x}=\frac{2}{x^{2}}
$$

(Compare (12) with (10) above.) The Lévy measure $\nu$ gives that $W$ is a weakly 1-stable distribution.

The expression in (12) can be simplified to

$$
\mathbf{E} e^{i t W}=\exp (-\pi|t|+i t(-1-2 \ln 2-2 \ln |t|)) .
$$

The same result holds for $X_{e}\left(T_{n}\right)$.

Remark 3.1. We observe that the normalizing term we divide with in Theorem 3.1 and Theorem 3.2 above is $\frac{n}{\lg ^{2} n}$ and $\frac{n}{4 \ln ^{2} n}$ respectively and that the theorems state that $X_{v}\left(T_{n}\right)$ and $X_{e}\left(T_{n}\right)$ have the same asymptotic distribution 
after normalization, while one can easily show that $\mathbf{E}\left(X_{e}\left(T_{n}\right)\right)-\mathbf{E}\left(X_{v}\left(T_{n}\right)\right) \sim$ $C \frac{n}{\lg ^{2} n}$ (for some constant $C$ ). Consequently, in both cases the normalized $X_{v}(T)$ and $X_{e}(T)$ have the same asymptotic distribution but their first moments are not equal, which suggests that for a proof of Theorem 3.1 or Theorem 3.2 one can not use the method of moments.

The idea of Janson's proof in [14] is to approximate $X\left(T_{n}\right)$ by a sum of independent random variables derived from $\lambda_{v}$ and then apply a classical limit theorem for triangular arrays.

Obviously most records of the complete binary tree occur at levels close to the height of the tree that is $m=\lfloor\lg n\rfloor$, because almost all vertices are there. For the binary search tree most vertices are at height close to $2 \ln n$. Thus, most records occur at heights close to this level.

The idea of Janson's proof in [14] is to study subtrees $T_{i}, i=1, \ldots 2^{L}$ of size $n_{i}$, where their roots are at the smaller height $L$ which is close to $\lg \lg n$. He conditions on the root value $\lambda_{v}=y$ and lets $X_{n, y}$ be $X\left(T_{n}\right)-1$; that is the number of records in the tree given the root value except from the root value itself that always is a record. In particular he uses $X_{n_{i}, Y_{i}}$; the number of records in the subtree $T_{i}$ rooted at height $L$ given the root value $Y_{i}$.

In [14, Lemma 2.3] $X\left(T_{n}\right)$ is approximated as

$$
X\left(T_{n}\right)=\sum_{i=1}^{2^{L}} \mathbf{E}\left(X_{n_{i}, Y_{i}}\right)+o_{p}\left(\frac{n}{\lg ^{2} n}\right) .
$$

The notation $o_{p}\left(a_{n}\right)$ for positive integers $a_{n}$ (in (14) $\left.a_{n}=\frac{n}{\lg ^{2} n}\right)$ means $\frac{Z_{n}}{a_{n}} \stackrel{p}{\rightarrow} 0$ as $n \rightarrow \infty$ for random variables $Z_{n}$. To get an error term small as $o_{p}\left(\frac{n}{\lg ^{2} n}\right)$ in (14) one has to choose the lower bound of $L$ close to $\lg \lg n$ (or larger).

In [14, Lemma 2.1] Janson approximates $\mathbf{E}\left(X_{n, y}\right)$ (in particular used to get an approximation of $\left.\mathbf{E}\left(X_{n_{i}, Y_{i}}\right)\right)$ up to an error term that is small enough. By using this approximation and (14) above, one gets an approximation of $X\left(T_{n}\right)$ up to the error term $o_{p}\left(\frac{n}{\lg ^{2} n}\right)$ by a sum that depends on the random variables $\left\{Y_{i}, i=1, \ldots 2^{L}\right\}$ where $Y_{i}$ depend on $\left\{\lambda_{v}: h(v) \leq L\right\}$, see [14, equation (14)].

Using the structure of the tree that all levels are full up to the last one Janson gets a result of $\mathbf{E}\left(X_{n, y}\right)$ depending on the random parameter $y$ (and the fixed parameter $n)$, see [14, Lemma 2.2].

Using that $Y_{i}$ depend on $\left\{\lambda_{v}: h(v) \leq L\right\}$ Janson shows in [14, Lemma 2.4] how one can equally approximate $X\left(T_{n}\right)$ up to the error term $o_{p}\left(\frac{n}{\lg ^{2} n}\right)$ by a sum involving the parameters $\left\{\lambda_{v}: h(v) \leq L\right\}$ instead of $Y_{i}, i=1 \ldots, 2^{L}$. The expression he gets is 


\section{Theorem 3.3.}

$$
X\left(T_{n}\right)=\frac{n}{m}+L \frac{n}{m^{2}}-\frac{1}{m} \sum_{h(v) \leq L} n_{v} e^{-m \lambda_{v}}+\frac{2^{m+1}}{m^{2}}+o_{p}\left(m^{-2} n\right) .
$$

For the binary search tree I find a similar way to approximate $X\left(T_{n}\right)$ by a sum of conditional expected values obtained from a height $L$ that also is close to $\lg \lg n$ (or $\lg \ln n$ as is used for practical reasons) as was the case for the $L$ Janson uses for the complete binary tree in [14]. However, for the binary search tree one can not choose exactly the same height as is chosen in [14].

By letting $X\left(T_{n}\right)_{y}$ be $X\left(T_{n}\right)-1$ conditioned on the root label $\lambda_{\sigma}=y$, I define $\varphi\left(T_{i}, \Lambda_{i}\right):=\mathbf{E}\left(X\left(T_{i}\right)_{\Lambda_{i}} \mid T_{i}, \Lambda_{i}\right)$ as the conditional expected value given the tree $T_{i}$ and $\Lambda_{i}$ (where $T_{i}$ 's are the subtrees rooted at $v_{i}, \quad 1 \leq i \leq 2^{L}$ ). Since the subtree size $n_{i}$ of $T_{i}, i=1, \ldots 2^{L}$ is random, when studying $\varphi\left(T_{i}, \Lambda_{i}\right)$ as a function of $n_{i}$ it is natural to condition on $n_{i}$.

The result of Lemma 2.3 in The Paper is

$$
X\left(T_{n}\right)=\sum_{i=1}^{2^{L}} \varphi\left(T_{i}, \Lambda_{i}\right)+o_{p}\left(\frac{n}{\lg ^{2} n}\right) .
$$

Note that this is similar to (14) above as Janson gets for the complete binary tree. For Lemma 2.3 in The Paper to yield it is important that $L$ (the height for which the subtrees $T_{i}$ are rooted at) is chosen with a lowerbound which is close to $\lg \lg n$.

In contrast to the case of the complete binary tree that is deterministic, one does not know how many vertices there are at each level $j$ in the binary search tree since it is a random number. This explains why it is harder to estimate $\varphi\left(T_{i}, \Lambda_{i}\right)$, in Lemma 2.1 in The Paper than the corresponding $\mathbf{E}\left(X_{n, y}\right)$ in [14, Lemma 2.2] for the complete binary tree. However, as mentioned above in Subsection 1.2, there are several results of the structure of the tree, such that for a tree of size $n$ one knows most vertices are at height close to $2 \ln n$. These results are used frequently in the proof of Lemma 2.1.

Using (16) above and the approximation of $\varphi\left(T_{i}, \Lambda_{i}\right)$ in Lemma $2.1 \mathrm{in}$ The Paper, $X\left(T_{n}\right)$ can be expressed by a sum that depends on the random variables $\Lambda_{i}$ up to the error term $o_{p}\left(\frac{n}{\lg ^{2} n}\right)$.

From this approximation it follows immediately following Janson's proof of [14, Lemma 2.4] that $X\left(T_{n}\right)$ equally can be approximated up to $o_{p}\left(\frac{n}{\lg ^{2} n}\right)$ by a sum that does not involve the $\Lambda_{i}$ 's but the $\lambda_{v}$ 's. The reason why in the approximation of $X\left(T_{n}\right)$ one can change variables from $Y_{i}$ 's to $\lambda_{v}$ 's and from 
$\Lambda_{i}$ 's to $\lambda_{v}$ 's in the complete binary tree and the binary search tree respectively without changing the error term $o_{p}\left(\frac{n}{\lg ^{2} n}\right)$ is because the height $L$ in both cases is chosen with an upper bound close to $\lg \lg n$. For a larger value of $L$ one does not achieve the result of Lemma 2.4 in The Paper. Together with the lower bound of $L$ (that one gets in the proof of Lemma 2.3) one sees why $L$ has to be chosen close to $\lg \lg n$. The corresponding result to Theorem 3.3 above that is stated in Lemma 2.4 in The Paper is

Theorem 3.4. Choosing $L=\lfloor\beta \lg \ln n\rfloor$ for some $\beta \in\left(\frac{}{\lg 3-1}, 2\right)$,

$$
\begin{aligned}
X\left(T_{n}\right)=\sum_{i=1}^{2^{L}} \frac{n_{i}}{\ln n_{i}}- & \sum_{i=1}^{2^{L}} \frac{\Upsilon\left(T_{i}\right)}{4 \ln ^{2} n_{i}} \\
& -\frac{1}{2 \ln n} \sum_{h v \leq L} n_{v} e^{-(2 \ln n) \lambda_{v}}+\frac{n}{4 \ln ^{2} n}+o_{p}\left(\frac{n}{\ln ^{2} n}\right),
\end{aligned}
$$

where $\Upsilon\left(T_{i}\right)$ denotes the total path length of the tree $T_{i}$; that is the sum of all depths (heights) of the vertices in the tree.

As mentioned above $L$ in Theorem 3.4 is not the same $L$ as in Theorem 3.3 above but close to.

The sum in Theorem 3.3 is a sum of independent random variables (depending on the $\lambda_{v}$ 's). By normalizing properly according to Theorem 3.1 above, the proof Janson presents is completed by a classical result on convergence of sums of triangular null arrays to infinitely divisible distributions, see [17, Theorem 15.28]. A triangular array is a sequence of random variables $\xi_{n, j}, 1 \leq j \leq n$, so that the variables in each row $j$ are independent and identically distributed, while the variables in different rows are not independent. A null array is a triangular array $\xi_{n, j}, 1 \leq j \leq n$ such that $\xi_{n, j} \stackrel{p}{\rightarrow} 0$. A random variable has an infinitely divisible distribution, if and only if for all $n$ there is a triangular array $\xi_{n, j}, 1 \leq j \leq n$ such that for all $n$

$$
X \stackrel{d}{=} \sum_{j=1}^{n} \xi_{n, j},
$$

see e.g. [11, Section 9.4]. To prove Theorem 3.1 stated above Janson defines $\xi_{v}:=\frac{m n_{v}}{n} e^{-m \lambda_{v}}$ and then by normalizing $X\left(T_{n}\right)$ as follows he obtains (see [14, equation (16)])

$$
\begin{aligned}
& \frac{m^{2}}{n}\left(X_{v}\left(T_{n}\right)-\frac{n}{\lg n}-\frac{n \lg \lg n}{\lg ^{2} n}\right) \\
& =\{\lg n\}+L-\lg \lg n+2^{1-\{\lg n\}}-\sum_{h(v) \leq L} \xi_{v}+o_{p}(1),
\end{aligned}
$$


where as introduced above $\{x\}$ denotes the fractional part of $x$. Here $\left\{\xi_{v}\right\}$ form a null array which is used to get the asymptotic distribution of the normalized $X\left(T_{n}\right)$. By showing that three conditions of a null array $\left\{\xi_{v}\right\}$ are fullfilled, [17, Theorem 15.28] guarantees that there is a distribution $\xi$ so that the sum $\sum_{h(v) \leq L} \xi_{v} \stackrel{d}{\rightarrow} \xi$. The calculations one gets to show these conditions to be satisfied also give the characteristic function of $\xi$. In [14, Lemma 2.5] Janson proves that the necessary conditions of $\left\{\xi_{v}\right\}$ are fullfilled so that $[17$, Theorem 15.28] can be used to prove convergence of $\sum_{h(v) \leq L} \xi_{v}$. From this lemma using (19) above one easily gets the asymptotic distribution of $X\left(T_{n}\right)$ in Theorem 3.1.

For the binary search tree one needs a similar result as [14, Lemma 2.5] to be able to apply the classical theorem of triangular arrays that is used in [14]. After normalizing $X\left(T_{n}\right)$ according to Theorem 3.2 one gets a similar result as (19) above, see equation (41) in The Paper, where $\frac{2 \ln n}{n} e^{-2 \ln n \lambda_{v}}$ denoted by $\xi_{v}$ is similarly to the nullarray that is used in [14]. However, $\left\{\xi_{v}:=\frac{2 \ln n}{n} e^{-2 \ln n \lambda_{v}}\right\}$ is not a triangular array since in a binary search tree the $n_{v}$ 's are not independent (although they in fact are less dependent for vertices that are far from each other). This complicates things significantly so that it is harder and more technical to prove a result that corresponds to [14, Lemma 2.5].

Furthermore, Theorem 2.1 stated in The Paper is the result that is needed to use the "classical triangular array" theorem for finding the asymptotic distribution of the normalized $X\left(T_{n}\right)$. For the sake of independence I condition on the subtree sizes $n_{v}$ 's in $\left\{\xi_{v}\right\}$. The idea is to show that (after normalization) $X\left(T_{n}\right)$ conditioned on the $n_{v}$ 's converges in distribution to $W$ with an infinitely divisible distribution, which is not depending on the $n_{v}$ 's that are conditioned on. Consequently, after normalization also $X\left(T_{n}\right)$ unconditioned converges in distribution to $W$. To prove Theorem 2.1, which is done by showing a kind of "law of large numbers" using how the subtree sizes $n_{v}$ 's are distributed, is the most difficult part of The Paper. The proof is quite long including comprehensive calculations.

However, the method that Janson invented in [14] works for the binary search tree and presumably for other logarithmic trees as well. The project I am currently working on is trying to extend this method to work also for the larger class of "split trees" (discussed in Subsection 1.2 above) where the binary search tree is one example of such tree. Hopefully I can refer to many of the calculations for the binary search tree even if the class of split trees contains many different trees. In the subsection that follows I present some ideas how I intend to tackle this project. 


\subsection{Ideas and Preliminary Results on Random Records and Cuttings in Split Trees}

The split trees which are discussed in Subsection 1.2 constitute a subclass of trees in the bigger class of trees of logarithmic height. The advantage in analyzing split trees compared to other trees of logarithmic height is that there is a general definition of these trees given in Subsection 1.2. This is the reason why I only intend to consider the split trees when generalizing the result of the asymptotic distribution of $X\left(T_{n}\right)$ in The Paper. Hopefully I can present a result that includes many split trees; one might need some conditions on the $\mathcal{V}$-vector as is explained below.

The method used in The Paper should also work for other trees of logarithmic height as well (the complete binary tree in [14] is one example), since many of these trees have similar properties.

In the proof of Theorem 3.2 above that is Theorem 1.1 in The Paper, it is frequently used that many nodes in a binary search tree of size $n$ are close to $2 \ln n$. Similarly the result in [5] stated in Theorem 1.1 above implies that for a general split tree there is a depth $D_{n}=\frac{\ln n}{\mu}$ which most vertices are close to. (Note that this means that for the binary search tree $\frac{1}{\mu}=2$.)

The result I expect for the split trees is that the asymptotic distribution of $X\left(T_{n}\right)$ is of the type

$$
\frac{\left(X_{v}\left(T_{n}\right)-\frac{n}{\left(\frac{\ln n}{\mu}\right)}-\frac{C n \ln \ln n}{\ln ^{2} n}\right)}{\frac{n}{\left(\frac{\ln ^{2} n}{\mu^{2}}\right)}} \stackrel{d}{\rightarrow}-W,
$$

where $W$ has an infinitely divisible distribution and $C$ is a constant (which I don't know yet) depending on the tree. I conjecture $W$ is also weakly 1-stable, but this I am not sure of even if I have done calculations that strengthen this hypothesis.

I expect the lemmas and Theorem 2.1 that are used to prove the main theorem, Theorem 1.1 in The Paper, all can be generalized to work for split trees in general. Because the trees are random it seems natural to keep many notations involving for example conditional expected values and conditional variances introduced in The Paper, in particular the notations of $\varphi\left(T_{i}, \Lambda_{i}\right):=$ $\mathbf{E}\left(X\left(T_{i}\right)_{\Lambda_{i}} \mid T_{i}, \Lambda_{i}\right)$ and $\psi\left(T_{i}, \Lambda_{i}\right):=\operatorname{Var}\left(X\left(T_{i}\right)_{\Lambda_{i}} \mid T_{i}, \Lambda_{i}\right)$. I expect the $T_{i}{ }^{\prime} \mathrm{s}$ are rooted at a height $L$ close to $\lg \lg n$ as for the complete binary tree and the binary search tree. For simplicity in the definition of $L$ in The Paper (which occurs for example in Lemma 2.3 and Theorem 2.1), $\lg \ln n$ is used for the binary search tree instead of $\lg \lg n$ that is used in [14] for the complete binary 
tree. Recall from Subsection 1.2 that branch factor $b$ means each vertex have exactly $b$ children. In a general split tree of branch factor $b$, I suggest that a suitable choice of $L$ corresponding to the choice of $L$ for the binary search tree (where $b=2$ ) is $L=\left\lfloor\beta \log _{b} \ln n\right\rfloor$, where $\beta$ is chosen in interval depending on the type of split tree. I argue below why I think it is reasonable that the height $L=\left\lfloor\beta \log _{b} \ln n\right\rfloor$ also occurs in the proof for a general split tree as in the particular case of the binary search tree.

The approximation of $\varphi\left(T_{i}, \Lambda_{i}\right)$ for the subtrees $T_{i}$ that are rooted at $v_{i}$ in Lemma 2.1 in The Paper is found by using that

$$
\varphi\left(T_{i}, \Lambda_{i}\right)=\sum_{v \neq v_{i}} \frac{1-e^{-h_{i}(v) \Lambda_{i}}}{h_{i}(v)}=\sum_{j} \sum_{h_{i}(v)=j} \frac{1-e^{-j \Lambda_{i}}}{j},
$$

where $h_{i}(v)$ is the height of $v$ in the subtree $T_{i}$ and $I_{v}$ is the indicator that $\lambda_{v}$ is the smallest of all values attached to the nodes in the unique path from $v$ to the root $v_{i}$. (This is equation (8) in The Paper.)

I use in particular that for a binary search tree of size $n$ there are few nodes that are not in a strip $2 \ln n-\ln ^{0.6} n \leq h(v) \leq 2 \ln n+\ln ^{0.6} n$. This fact is used several times in the proof of Theorem 3.2 above that is Theorem 1.1 in The Paper.

In general, finding the approximation of $\varphi\left(T_{i}, \Lambda_{i}\right)$ for split trees one can use (21) above and probably use the same type of proof as in The Paper. First one finds the Taylor expansion of $\sum_{v \neq v_{i}} \frac{1}{h_{i}(v)}$ about $\frac{\ln n_{i}}{\mu}\left(T_{i}\right.$ is of size $\left.n_{i}\right)$ up to an error term that is $\mathcal{O}_{L^{1}}\left(\frac{n_{i}}{\ln ^{2 \cdot 2} n_{i}}\right)$, this error term is used frequently in the paper. Then by using that many nodes are close to $\frac{\ln n_{i}}{\mu}$ one shows that multiplying this expansion by $\left(1-e^{-\left(\frac{\ln n_{i}}{\mu}\right) \Lambda_{i}}\right)$ gives an expression of $\varphi\left(T_{i}, \Lambda_{i}\right)$ up to $\mathcal{O}_{L^{1}}\left(\frac{n_{i}}{\ln ^{2.2} n_{i}}\right)$.

In particular, from (4) in Theorem 1.1 above, one sees there are few vertices that are not in a strip $\frac{\ln n_{i}}{\mu}-\ln ^{0.6} n_{i} \leq h_{i}(v) \leq \frac{\ln n_{i}}{\mu}+\ln ^{0.6} n_{i}$.

Also a sharper profile estimate is used in the proof when proving (21) above, but it seems like this profile result is sharper than one needs which I briefly explain here. The sharp profile estimation is used in The Paper to approximate the third term $\frac{\sum_{v}\left(h_{i}(v)-2 \ln n_{i}\right)^{2}}{8 \ln ^{3} n_{i}}$ in equation (11) in The Paper. However, I could manage with the result of equation (4) in Theorem 1.1 above using that the expected number of $\left(h_{i}(v)-2 \ln n_{i}\right)^{2}$ is close to the variance of $h_{i}(v)$ which one gets from the denominator in (4) (that is the square root of the variance) and which for the binary search tree of size $n_{i}$ is close to its expected value $2 \ln n_{i}$. One gets that for a binary search tree, the third term is $\frac{n_{i} 2 \ln n_{i}}{8 \ln ^{3} n_{i}}=\frac{n_{i}}{4 \ln ^{2} n_{i}}$ with the error term $\mathcal{O}_{L^{1}}\left(\frac{n_{i}}{\ln ^{2.2} n_{i}}\right)$. For a general split tree one gets by the same type of argument that the corresponding term is $\frac{\sigma^{2} n_{i}}{\ln ^{2} n_{i}}$ 
with the error term $\mathcal{O}_{L^{1}}\left(\frac{n_{i}}{\ln ^{2.2} n_{i}}\right)$, where $\sigma^{2}$ is defined in (2) above. I use the sharp profile estimate also to show equation (15) in The Paper (which I do by showing $\left.(16)+(17)=\mathcal{O}_{L^{1}}\left(\frac{n_{i}}{\ln ^{2.2} n_{i}}\right)\right)$ that is the part where I show that I get an expression of $\varphi\left(T_{i}, \Lambda_{i}\right)$ that is good enough by multiplying the expansion of $\sum_{v \neq v_{i}} \frac{1}{h_{i}(v)}$ by $\left(1-e^{-\left(2 \ln n_{i}\right) \Lambda_{i}}\right)$. However, also here one could manage to show this by only using the weak result of the profile that there are "few nodes" outside $2 \ln n_{i}-\ln ^{0.6} n_{i} \leq h_{i}(v) \leq 2 \ln n_{i}+\ln ^{0.6} n_{i}$. By analogy for a general split tree to show an equation that correspond to equation (15) in The Paper it is enough to use that one only have to consider the vertices in $\frac{\ln n_{i}}{\mu}-\ln ^{0.6} n_{i} \leq h_{i}(v) \leq \frac{\ln n_{i}}{\mu}+\ln ^{0.6} n_{i}$ since the vertices outside this strip are few and can be ignored, and thus there is no need for a sharper profile estimate to prove this lemma.

Proving Lemma 2.2 that is the upperbound of $\psi\left(T_{i}, \Lambda_{i}\right)$ in The Paper, I use that

$$
\psi\left(T_{i}, \Lambda_{i}\right)=\sum_{v, w \in T_{i}} \operatorname{Cov}\left(I_{v}, I_{w}\right)
$$

where $I_{v}$ is the indicator function in (21) above. The idea of the proof is to first take the sum of covariances over "good pairs" $(v, w)$ in $T_{i}$ (of size $n_{i}$ ) since they have small covariances and also the sum of them is small. These pairs are defined as those that are in the strip $2 \ln n_{i}-\ln ^{0.6} n_{i} \leq h_{i}(v) \leq 2 \ln n_{i}+\ln ^{0.6} n_{i}$ (this strip as was mentioned is frequently used in The Paper), the other criterion is that they also have their last common ancestor at height $h \leq \alpha \ln n_{i}$ for some constant $\alpha$. The "bad pairs" (those that are not good) have a larger covariance, but this does not matter since they are few again using the fact that there are few vertices outside the strip $2 \ln n_{i}-\ln ^{0.6} n_{i} \leq h_{i}(v) \leq 2 \ln n_{i}+\ln ^{0.6} n_{i}$. To prove Lemma $2.2 \mathrm{I}$ also use the first and second moment of the subtree sizes $n_{i}$, see equation (23) in The Paper. In a tree of size $n$ one calculates (in the proof of Lemma $2.2 n_{i}$ is used instead of $n$ since the $T_{i}$ 's are considered) using the subtree sizes expressed as random variables, $\mathbf{E}\left(n_{v}\right)$ for a vertex $v$ at height $j$ (where $j$ is not too large) as very close to the upperbound $\frac{n}{2^{j}}$. This can also be obtained without calculations: At level $j$ there are $2^{j}$ vertices and the sum of the subtree sizes has to be close to $n$ for subtrees rooted at $j$ (if $j$ is not too large there are just a few vertices below this level). This implies that since the subtree sizes are identically distributed, the mean is just the mean value of the sum of nodes above level $j$ and thus the mean is close to $\frac{n}{2^{j}}$.

For a general split tree of branch factor $b$, I expect one could argue in the same manner. There is a strip $\frac{\ln n}{\mu}-\ln ^{0.6} n \leq h_{i}(v) \leq \frac{\ln n}{\mu}+\ln ^{0.6} n$ where most vertices are in the subtree $T_{i} i=1, \ldots b^{L}$. Thus, the good pairs $(v, w)$ can be defined as those that are in this strip together with the criterion that $(v, w)$ 
have their last common ancestor at height $h \leq \alpha \ln n_{i}$ for some constant $\alpha$ as for the binary search tree. Also the first and second moment, can be calculated, since as mentioned in Subsection 1.2 the subtree sizes $n_{v}$ in a split tree given the total size $\left(T_{i}\right.$ is here a tree of size $\left.n_{i}\right)$ can be expressed as random variables using the split vector $\mathcal{V}$. Comparing with the binary search tree one sees that in a general split tree of size $n$ and branch factor $b$ the first moment $\mathbf{E}\left(n_{v}\right)$ for a vertex $v$ at height $j$ (where $j$ is not too large) must be close to $\frac{n}{b^{j}}$ using that the subtree sizes are equally distributed and that at height $j$ there are $b^{j}$ vertices. (Even if a stronger result of the second moment is used in The Paper for the binary search tree, one in fact only needs a rough estimate of this for proving this lemma.) In Lemma 2.3 in The Paper the result of Lemma 2.2 is used to show that $X\left(T_{n}\right)$ can be approximated by

$$
X\left(T_{n}\right)=\sum_{i=1}^{2^{L}} \varphi\left(T_{i}, \Lambda_{i}\right)+o_{p}\left(\frac{n}{\lg ^{2} n}\right),
$$

where $L$ is close to $\lg \ln n$. In the proof I use equation (23) in The Paper to get $\sum_{i=1}^{2^{L}} \mathbf{E}\left(n_{v}^{2}\right)=\mathcal{O}\left(2^{L} \frac{n^{2}}{3^{L}}\right)=o\left(\frac{n^{2}}{\ln n}\right)$. In general for split trees with branch factor $b$ one may argue that choosing the constant $\beta$ in $L=\left\lfloor\beta \log _{b} \ln n\right\rfloor$ large enough one gets

$$
\sum_{i=1}^{b^{L}} \mathbf{E}\left(n_{v}^{2}\right)=\mathcal{O}\left(\frac{n^{2}}{\ln n}\right) .
$$

At height $L$ the distribution of $n_{v}$ is close to $n X$ where $X$ is a product of $L$ independent random variables $\left\{Z_{i}, i=1, \ldots, L\right\}$ with $Z_{i} \in[0,1]$. Thus, $n_{v}^{2}=n^{2} X^{2}$ and $\mathbf{E}\left(Z_{i}^{2}\right)<\mathbf{E}\left(Z_{i}\right)=\frac{1}{b}$ implies there is an $\epsilon>0$ such that

$$
\mathbf{E}\left(X^{2}\right)=\prod_{i=1}^{L} \mathbf{E}\left(Z_{i}^{2}\right) \leq \frac{1}{(b+\epsilon)^{L}} .
$$

This implies that if $\beta$ in $L=\left\lfloor\beta \log _{b} \ln n\right\rfloor$ is large enough one gets (22) above. From Lemma 2.3 in The Paper I approximate $X\left(T_{n}\right)$ up to $o_{p}\left(\frac{n}{\ln ^{2} n}\right)$ as a sum depending on $\Lambda_{i}$, see equation (35) in The Paper. From this (as mentioned in Subsection 3.1) it is easy to show by using Janson's calculations in the proof of [14, Lemma 2.4] that $X\left(T_{n}\right)$ also can be approximated up to $o_{p}\left(\frac{n}{\ln ^{2} n}\right)$ as a sum depending on $\left\{\lambda_{v}, h(v) \leq L\right\}$ instead of $\left\{\Lambda_{i}, i=1 \ldots L\right\}$. The proof of Lemma 2.4 in The Paper uses that $\mathcal{O}\left(\frac{2^{L} \ln ^{4} m}{m^{2}}\right)=o(1)$, where $m=2 \ln n$ is the "central" height which most vertices are close to. Thus, it is important that $L<2 \lg \ln n$. In general for split trees of branch factor $b$ it seems likely that 
one can achieve a similar result as Lemma 2.4 in The Paper again using the proof of [14, Lemma 2.4], since the calculations there are very general (that is the argument there also works for many other trees). However, for these trees because of the branch factor $b$ one needs $\mathcal{O}\left(\frac{b^{L} \ln ^{4} m}{m^{2}}\right)=o(1)$, where $m=\frac{1}{\mu} \ln n$ is the central region. Thus, one needs $L<2 \log _{b} \ln n$. Hence, together with the lower bound of $L$ one gets $L=\left\lfloor\beta \log _{b} \ln n\right\rfloor$ where $\beta \in(x, 2)$ for some $x$ depending on the type of split tree (or equivalently depending on the type of $\mathcal{V}$-vector), where one of course needs to show that $x$ satisfies $x<2$ to be able to choose a possible $L$. This is satisfied for many important split trees, but unfortunately not for all. However, if there is a $\beta \in(x, 2)$ one gets an expression of $X\left(T_{n}\right)$ (similar to the one in Lemma 2.4 in The Paper) that is

$$
\begin{aligned}
X\left(T_{n}\right)=\sum_{i=1}^{b^{L}} \frac{2 n_{i}}{\frac{1}{\mu} \ln n_{i}} & -\sum_{i=1}^{b^{L}} \frac{\Upsilon\left(T_{i}\right)}{\frac{1}{\mu^{2}} \ln ^{2} n_{i}} \\
& -\frac{1}{\frac{1}{\mu} \ln n} \sum_{h(v) \leq L} n_{v} e^{-\left(\frac{1}{\mu} \ln n\right) \lambda_{v}}+\frac{\sigma^{2} n}{\ln ^{2} n}+o_{p}\left(\frac{n}{\ln ^{2} n}\right),
\end{aligned}
$$

where $\mu$ and $\sigma^{2}$ are defined in equation (2) above. Note that (24) (except for possibly the total path length $\Upsilon\left(T_{i}\right)$ ) depends on the split tree parameters $b$ and $\mathcal{V}$ and not of the parameters $s, s_{0}$ and $s_{1}$.

The expression in Lemma 2.4 in The Paper is then used in Theorem 2.1 to show that the triangular array theorem in [17, Theorem 15.28] can be used to find the distribution $W$ which $X\left(T_{n}\right)$ (after normalization) converges to. Theorem 2.1 is as mentioned above the hardest result to show in The Paper, and is shown by using a "law of large numbers" using the subtree sizes in a binary search tree represented as random variables. Since there is such a representation of subtree sizes also for general split trees, I hope to be able to extend also this theorem using (24).

\section{Conclusions}

The proof of Theorem 1.1 in The Paper shows that the method by approximating $X\left(T_{n}\right)$ by a sum of independent random variables derived from $\left\{\lambda_{v}\right\}$ that is used in [14] can be modified so that it also allows finding the asymptotic distribution of $X\left(T_{n}\right)$ (normalized) for the binary search tree. The results obtained for the binary search tree are similar to the lemmas that are presented in [14]) for the complete binary tree. However, the proofs of the results for the binary search tree are more complicated and require more 
extensive calculations due to the random rather than deterministic nature of this tree. At the same time the randomness makes the result for the binary search tree nicer than that for the complete binary tree. Thus, after normalization there is a true asymptotic distribution of $X\left(T_{n}\right)$ for the binary search tree, while for the complete binary tree there is no true asymptotic distribution but a family of limits of different subsequences.

Furthermore, the discussion and preliminary results in Subsection 3.2 strengthen the hypothesis that the method can also be extended to find the asymptotic distribution of $X\left(T_{n}\right)$ even for the larger class of split trees. See in particular equation (24) above, which is an approximation of $X\left(T_{n}\right)$ for split trees by a sum derived from $\left\{\lambda_{v}\right\}$.

\section{Acknowledgements}

First and foremost, I would like to thank my supervisor Professor Svante Janson for being the perfect supervisor, for introducing me to this problem area, for generously sharing his tremendous knowledge with me, for always having time for me and for carefully reading and correcting this licentiate thesis. I also greatly appreciate that he has always encouraged me to find the solutions myself.

My second supervisor Professor Sven Erick Alm is gratefully acknowledged for his interest in my work and for carefully reading and revising the text.

I also want to thank Professor Allan Gut for his encouragement and support; his book "Probability: A Graduate Course" has also been very helpful for some of my calculations.

Finally I want to thank all my PhD student friends, especially Johanna and the other members of our female network "Lilla $\mu$ ", Henrik, Johan, Micke and Niclas for great companionship, encouragement and support during my time at the Department of Mathematics at Uppsala university. I also like to thank my roommate Henrik (Renlund) for valuable discussions, as well as Johan (Björklund) and Olov Wilander for helping me with the figures and layout of this thesis. 


\section{Bibliography}

[1] R. M Blumenthal, Excursions of Markov Processes. Birkhäuser Boston, 2002 .

[2] B. Bollobás Random Graphs. Academic Press, London, 1985.

[3] B. Chauvin, M. Drmota, and J. Jabbour Hattab, The profile of binary search trees. Ann. Applied Probab. 11, 2001, 1042-1062.

[4] L. Devroye, A note on the height of binary search trees. J. Assoc. Comput. Mach. 33, 1986, 489-498.

[5] L. Devroye, Universal limit laws for depths in random trees. Siam J. Comput. Vol 28, No 2, 1998, 409-432.

[6] L. Devroye, Branching processes and their applications in the analysis of tree structures and tree algorithms. Probabilistic Methods for Algorithmic Discrete Mathematics, ed. Habib, et al. Springer Series on Algorithms and Combinatorics, vol. 16, Springer, Berlin, 1998, 249-314.

[7] L. Devroye, Applications of Steins method in the analysis of random binary search trees. Steins method and Applications, ed. Chen and Barbour, Institute for Mathematical Sciences Lecture Notes Series, vol. 5, World Scientific Press, Singapore, 2005, 247-297.

[8] M. Drmota, Analytic approach to the height of the binary search tree. Technical report, University of Vienna 1997.

[9] M. Drmota, A. Iksanov, M. Moehle and U. Roesler, A limiting distribution for the number of cuts needed to isolate the root of a random recursive tree. Preprint, 2006.

[10] W. Feller, An Introduction to Probability Theory and Its Applications. Volume II 2nd edition, Wiley, New York, 1971. 
[11] A. Gut, Probability: A Graduate Course, Springer Texts in Statistics, Springer, New York, 2005.

[12] A. Iksanov, M. Moehle, A probabilistic proof of a weak limit law for the number of cuts needed to isolate the root of a random recursive tree. Preprint, 2006.

[13] J. A. Fill and S. Janson, Quicksort asymptotics. J. Algorithms 44, 2002, $4-28$.

[14] S. Janson, Random records and cuttings in complete binary trees. Mathematics and Computer Science III, Algorithms, Trees, Combinatorics and Probabilities (Vienna, 2004,) Birkhäuser, Basel/Switzerland, 2004, 241-253.

[15] S. Janson, Random cuttings and records in deterministic and random trees. Random Struct. Alg. 29, 2006, 139-179.

[16] S. Janson, T. Łuczak, A. Rucinski, Random Graphs. Wiley Interscience Series in Discrete Mathematics and Optimization, Wiley, New York, 2000 .

[17] O. Kallenberg, Foundations of Modern Probability. 2nd edition, Springer Verlag, Reading, Mass., 2002.

[18] D. E. Knuth, The Art of Computer Programming. Volume 1: Fundamental Algorithms. 3rd edition, Addison Wesley, Reading Mass., 1997.

[19] D. E. Knuth, The Art of Computer Programming. Volume 3: Sorting and Searching. Addison-Wesley, New York, 2002.

[20] H. Mahmoud and B. Pittel, On the most probable shape of a search tree grown from a random permutation. SIAM Journal on Algebraic and Discrete Methods 5, 1984, 69-81.

[21] A. Meir and J. W. Moon, Cutting down random trees. J. Australian Math. Soc. 11, 1970, 313-324.

[22] A. Panholzer, Cutting down very simple trees. Submitted, 2003.

[23] J. M. Robson, The height of binary search trees. The Australian Computer Journal 11, 1979, 151-153. 


\section{Part II}

Manuscript, to be Submitted 


\title{
Random Records and Cuttings in Binary Search Trees
}

\author{
Cecilia Holmgren
}

October 11, 2007

\begin{abstract}
We study the number of records in a random binary search tree on $n$ randomly labelled vertices. Equivalently the number of random cuttings required to eliminate a random binary search tree can be studied. After normalization the distribution is shown to be asymptotically 1-stable.
\end{abstract}

\section{Introduction}

\subsection{Preliminaries}

Given a rooted tree $T$ with $n$ nodes, let each vertex $v$ (one may alternatively consider edges) have a random value $\lambda_{v}$ attached to it, and assume that these values are i.i.d. with a continuous distribution. We say that the value $\lambda_{v}$ is a record if it is the smallest value in the path from the root to $v$. Let $X_{v}(T)$ denote the (random) number of records. It is easy to see that the distribution of $\lambda_{v}$ does not matter, that is one can choose it as any continuous distribution, or alternatively let the values $\lambda_{v}$ be a random permutation of $\{1,2, \ldots, n\}$, see $[12,11]$.

The same random variable appears when we consider cuttings of the tree $T$ (that is cuttings and records give random variables with the same distribution) defined as follows, see e.g. [18, 12]: Make a random cut by choosing one vertex at random. Delete this vertex so that the tree separates into two parts and keep only the part containing the root. Continue recursively until the root is cut. Then the total (random) number of cuts made is $X_{v}(T)$. The proof of this equivalence uses a natural coupling argument, see $[12,11]$.

A binary search tree is a binary tree in which each node is linked to a key, where the keys are drawn from a totally ordered finite set, say $\{1,2, \ldots, n\}$. The keys are drawn in a random order from the set until the set is exhausted. 
Each possible draw order corresponds to a permutation of the elements in the set (then each permutation is equally likely). The first key is linked to the root. The next key is placed at the left child of the root if it is smaller than the key of the root, and it is placed at the right child of the root if it is larger than the key of the root. In this way we proceed progressively, inserting key by key. When all keys are placed one gets a binary tree on $n$ vertices, such that the keys of the left subtree of any given node $x$ are smaller than the key of $x$, and the keys of the right subtree are larger than the key of $x$. See e.g. [1, 4].

In [12] the asymptotic distributions are found for random trees that can be constructed as conditioned Galton-Watson trees, e.g. labelled trees and random binary trees. There the proof relies on the fact that the method of moments could be used.

For the complete binary tree it is impossible to use the method of moments, see [11]. Therefore Janson introduced another strategy, which is to approximate $X_{v}(T)$ by a sum of independent random variables derived from $\lambda_{v}$, and then apply a classical limit theorem for triangular arrays. From the proof it is easy to see that the method also works for all complete $d$-ary trees as well.

In this paper we consider the case of binary search trees. These belong to the family of the so called $\log n$ trees, that are trees with height $\mathcal{O}(\log n)$. These trees have similar properties to the deterministic (not random) complete binary tree considered in [11]. The height in a complete binary tree is $\left\lfloor\log _{2} n\right\rfloor$, also the height in the $\log n$ tree is $\mathcal{O}(\log n)$ and the profile in the complete binary tree has most nodes at height $\left\lfloor\log _{2} n\right\rfloor$ compared to $\sim C \log n$ ( $C$ constant) for the $\log n$ tree.

\subsection{Useful facts about binary search trees}

In this section some of the most important facts and results of the binary search tree are collected for later use.

\subsubsection{Properties}

We say that an event $A_{n}$ happens asymptotically almost surely (a.a.s.) if $\mathbf{P}\left(A_{n}\right) \rightarrow 1$ as $n \rightarrow \infty$, see [13].

The height is a.a.s. $c_{1} \ln n+\mathcal{O}(\ln \ln n)\left(\right.$ where $\left.c_{1}=4.31107 \ldots\right)$. Also a.a.s. up to $c_{2} \ln n$ (where $c_{2}=0.3711 \ldots$ ) all levels are full. Most nodes are at height $2 \ln n+\mathcal{O}(\sqrt{\ln n})$ and the peak around the "belly" is Gaussian. (See e.g $[2,4,1,5]$.$) See figure 1$. 


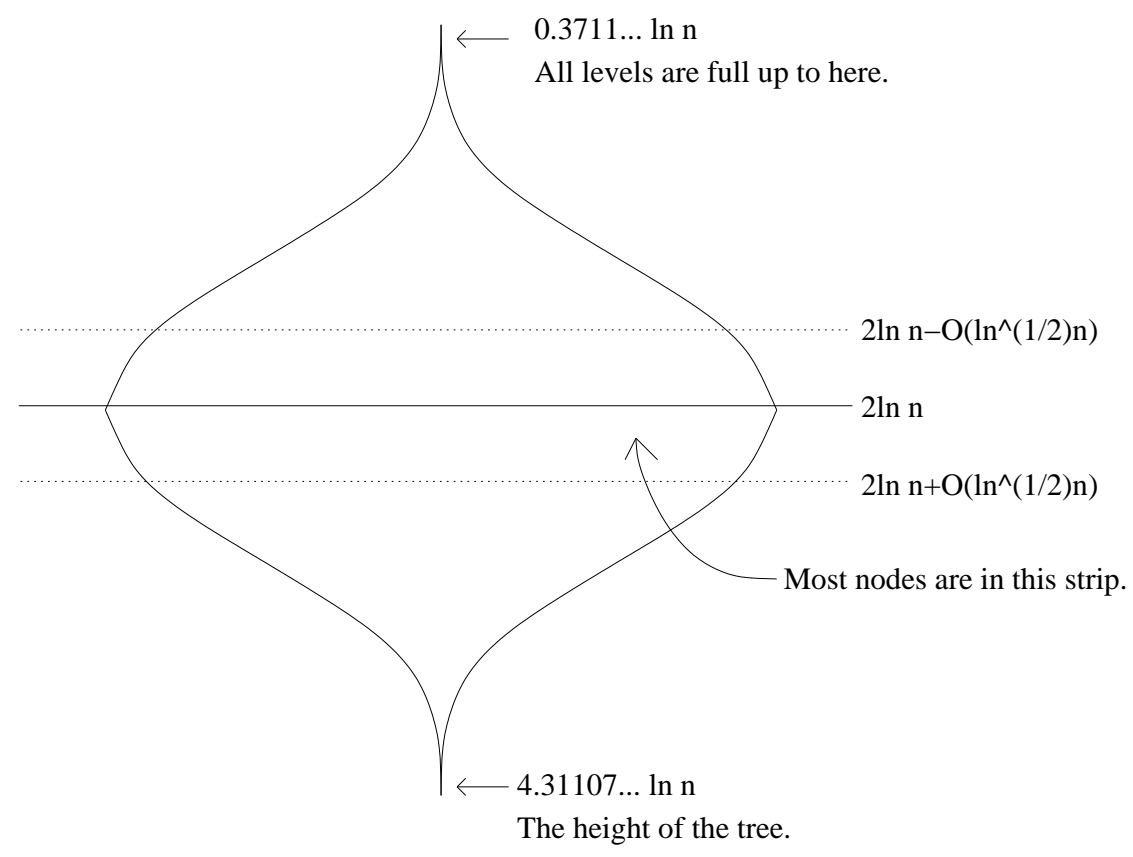

Figure 1: This figure illustrates the shape of the binary search tree. The root is at the top. The horizontal width represents the number of nodes at each level. Most nodes are in a strip of width $\mathcal{O}(\sqrt{\ln n})$ about $2 \ln n$.

\subsubsection{Quicksort}

Quicksort is one of the most used sorting algorithms because it is so fast and simple. It is the standard sorting algorithm in Unix systems, and is used for sorting an array of $n$ distinct numbers. The Quicksort algorithm can be described as follows:

If $n=0$ or $n=1$ there is nothing to do (the elements are sorted). If $n \geq 2$, then pick a number uniformly at random from the list of $n$ numbers. Compare the other numbers to it (smaller or larger) to get two subsets of numbers. Next apply the same procedure to each of these two sets if they contain more than one element. Thus, we end up with a sorted list of the original numbers.

It is easy to see that the random tree that one gets from Quicksort is a random binary search tree, and this is one reason why these trees are very interesting, see e.g. $[4,7]$.

Let $L_{n}$ denote the number of comparisons required. Then it is easy to see that $L_{n}$ satisfies

$$
L_{n} \stackrel{d}{=} L_{U_{n}-1}+L_{n-U_{n}}^{*}+n-1, \quad n \geq 1
$$


where $U_{k}$ is uniformly distributed on the set $\{1,2, \ldots, k\}, \stackrel{d}{=}$ denotes equality in distribution and $L_{j}^{*} \stackrel{d}{=} L_{j}$ and $U_{n} ; L_{0}, L_{1}, \ldots L_{n-1} ; L_{0}^{*}, L_{1}^{*}, \ldots L_{n-1}^{*}$ are all independent.

This random variable $L_{n}$ is equal to the so called total path length (we denote by $\Upsilon(T)$ the total path length of the binary search tree $T$ ), which equals the sum of the heights (distances to the root) for all $n$ nodes in the (binary search) tree. This random variable has been extensively studied, see e.g. $[7,16,17]$. Two easily established facts are the expected value of $L_{n}$ see e.g. [15] and the variance of $L_{n}$, see [17, Exercise 6.2.2-8]:

$$
\begin{aligned}
& \mathbf{E} L_{n}=2(n+1) H_{n}-4 n=2 n \ln n+n(2 \gamma-4)+2 \ln n+2 \gamma+1+\mathcal{O}\left(\frac{1}{n}\right), \\
& \operatorname{Var} L_{n}=\sigma^{2} n^{2}-2 n \ln n+\mathcal{O}(n),
\end{aligned}
$$

where $H_{n}=\sum_{k=1}^{n} \frac{1}{k}$ (the $n$ 'th harmonic number), $\sigma^{2}=7-\frac{2}{3} \pi^{2}=0.42026 \ldots$ and $\gamma=0.5772 \ldots$ (Euler's constant).

\subsubsection{The subtree method}

There is a third way to generate the binary search tree that is very useful in many applications. We use this representation in Lemma 2.2 and Lemma 2.3 below. The method can be explained briefly as follows:

Associate with each node in the binary search tree $T_{n}$ (where $T_{n}$ denotes a binary search tree with $n$ vertices) the size of the subtree rooted at that node. This means that the root of $T_{n}$ has value $n$. Since the rank of the root element is equally likely to be $\{1, \ldots, n\}$, the random number $N$ of nodes in the left subtree of the root of $T$ is uniformly distributed on $\{0, \ldots, n-1\}$. It follows easily that $N \stackrel{d}{=}\lfloor n U\rfloor$ where $U$ is uniformly distributed on $[0,1]$. The size of the right subtree is $n-1-N$ which is distributed as $\lfloor n(1-U)\rfloor$. Now all subtree sizes in the tree can be explained similarly by proceeding in the same manner by introducing independent uniform $[0,1](U)$ random variables. See e.g. [3]. Equivalently this means that starting from the root (which we give the subtree value $n$ ) there is an independent uniform random variable $\mathcal{U}_{v}$ associated with each node $v$, such that if we let the right and left child of $v$ be $v_{r}$ and $v_{l}$ respectively and $v$ has subtree value $V$, then $v_{r}$ and $v_{l}$ have subtree value $\left\lfloor V \mathcal{U}_{v}\right\rfloor$ and $\left\lfloor V\left(1-\mathcal{U}_{v}\right)\right\rfloor$ respectively. This representation of uniform random variables gives that the subtree size of any node $v$ at height $j$ is distributed as

$$
\left\lfloor\ldots\left\lfloor\left\lfloor n U_{1}\right\rfloor U_{2}\right\rfloor \ldots U_{j}\right\rfloor
$$

where $U_{r}, r=1, \ldots j$, are i.i.d. uniform random variables given by the nodes in the unique path from vertex $v$ to the root vertex. Thus, the value $n$ and 
the uniform random variables associated to the nodes give all subtree sizes of the tree. For later use to see that a representation of uniform random variables as in (1) is a representation of the subtree size rooted at vertex $v$, we give a vertex index to these i.i.d. uniform random variables letting $U_{r, v}:=U_{r}$ so that the subtree size of $v$ at height $j$, is

$$
\left\lfloor\ldots\left\lfloor\left\lfloor n U_{1, v}\right\rfloor U_{2, v}\right\rfloor \ldots U_{j, v}\right\rfloor .
$$

This means e.g. for the right child vertex $v_{r}$ (at height $j+1$ ) of a vertex $v$ at height $j, U_{j+1, v_{r}}=\mathcal{U}_{v}$ and for the left child vertex $v_{l}, U_{j+1, v_{l}}=1-\mathcal{U}_{v}$.

\subsection{The Main Theorem}

Theorem 1.1. Suppose that $n \rightarrow \infty$. Then,

$$
\left(X_{v}\left(T_{n}\right)-\frac{n}{2 \ln n}-\frac{n \ln \ln n}{2 \ln ^{2} n}\right) / \frac{n}{4 \ln ^{2} n} \stackrel{d}{\rightarrow}-W,
$$

where $W$ has an infinitely divisible distribution with characteristic function

$$
\mathbf{E} e^{i t W}=\exp (-\pi|t|+i t(-1-2 \ln 2-2 \ln |t|)) .
$$

The same result holds for $X_{e}\left(T_{n}\right)$.

Remark 1.1. In the proof of (3) that follows below we get

$$
\mathbf{E} e^{i t W}=\exp \left(i(-3-2 \ln 2+2 \gamma) t+\int_{0}^{\infty}\left(e^{i t x}-1-i t x \mathbf{1}[x<1]\right) d \nu(x)\right),
$$

where $(\gamma=0.577 \ldots$ is the Euler constant) and the Lévy measure $\nu$ is supported on $(0, \infty)$ and has density

$$
\frac{d \nu}{d x}=\frac{2}{x^{2}}
$$

We can simplify the expression in (5) to get (4) above. The Lévy measure $\nu$ gives that $W$ is a weakly 1 -stable distribution. That a distribution of a random variable $Z$ is $\alpha$-stable for $\alpha \in(0,2]$ means that for a sequence of i.i.d. random variables $Z_{k}, k \geq 1$, (such that for all $k, Z_{k} \stackrel{d}{=} Z$ ), then $\sum_{k=1}^{n} Z_{k} \stackrel{d}{=} c_{n} Z+d_{n}$ for all $n$, where $c_{n}=n^{\frac{1}{\alpha}}$. The distribution is strictly stable if for all $n, d_{n}=0$ and weakly stable otherwise. See e.g. [8, Section XVII.3]. 
Remark 1.2. We note as in [11] that even if most records occur at height close to $\sim 2 \ln n$ where most of the vertices are, from Lemma 2.4 and the proof of Theorem 2.1 it follows that most of the random fluctuations of $X_{v}\left(T_{n}\right)$ can be explained by the values at heights close to $\ln \ln n$. As explained in [11], a few values at these heights are so small that they reduce a large number of records among their descendants. Vertices of smaller height are too few and so there is usually not any sufficiently small value among them. Vertices of larger height affect only a small subtree.

Remark 1.3. For all rooted trees $T_{n}$ (see $[12,11]$ ), $\mathbf{E} X_{v}\left(T_{n}\right)=\sum_{v} \frac{1}{h(v)+1}$ and for the edge version $\mathbf{E} X_{e}\left(T_{n}\right)=\sum_{v \neq \sigma} \frac{1}{h(v)}$ (where $\sigma$ is the root). For random trees $\mathbf{E}\left(X_{v}\left(T_{n}\right) \mid T_{n}\right)$ (or $\left.\mathbf{E}\left(X_{e}\left(T_{n}\right) \mid T_{n}\right)\right)$ is the conditional expected value given the tree $T_{n}$ thus $\mathbf{E}\left(X_{v}\left(T_{n}\right)\right)=\mathbf{E}\left(\sum_{v} \frac{1}{h(v)+1}\right)$.

Thus, for the binary search tree (compare to [11, Remark 1.3] ),

$$
\mathbf{E}\left(X_{e}\left(T_{n}\right)\right)-\mathbf{E}\left(X_{v}\left(T_{n}\right)\right)=\mathbf{E}\left(\sum_{v \neq \sigma} \frac{1}{h(v)(h(v)+1)}\right) \sim C \frac{n}{\log ^{2} n},
$$

for some constant $C>0$, while there is no similar difference in the limit distribution; see Theorem 1.1 above. As noted for the complete binary tree [11], this is explained by the fact that the expected value is affected by the unlikely event that a node close to the root has a very small value which reduces the number of records among its descendants significantly. This behaviour suggests it is impossible to use the method of moments to find the record distribution for the binary search tree as one could do for the Galton Watson trees. Instead we intend to use similar methods as were used for the complete binary tree, see [11].

Remark 1.4. Presumably the method that is used here should also work for many other trees of logarithmic height as well, and thus the limiting distribution for these trees should also be infinitely divisible and probably also weakly stable. This turns out to be the case for the random recursive tree (that is a logarithmic tree) where the limiting distribution of $X\left(T_{n}\right)$ was recently found to be weakly stable, see [6, Theorem 1.1] and [10, Theorem 1.1]. Note, that also the normalization for this tree is similar to the one in (3), which is not surprising from the proof of Theorem 1.1 that follows below. The proof of [6, Theorem 1.1] uses generating functions while the proof of [10, Theorem 1.1] is more probabilistic. However, in both of these proofs $X\left(T_{n}\right)$ is considered as the cutting down random variable instead of the record random variable that is considered in this paper and also the methods they use differ completely from the methods that we use here to find the limiting distribution of $X\left(T_{n}\right)$ for the binary search tree. 


\section{Proofs}

We use the usual notations lg for the 2-logarithm and ln for the $e$-logarithm, and let $\{x\}=x-\lfloor x\rfloor$ denote the fractional part of a real number $x$. We treat the case $X_{v}\left(T_{n}\right)$ in Theorem 1.1 in detail and then indicate why the same result holds for $X_{e}\left(T_{n}\right)$ too. From now on since it is clear that we consider the vertex model we just write $X\left(T_{n}\right)$. First let $X\left(T_{n}\right)_{y}$ be $X\left(T_{n}\right)-1$ conditioned on the root label $\lambda_{\sigma}=y$ (that is the number of records in $T_{n}$ given the root value $\lambda_{\sigma}=y$ except from $\lambda_{\sigma}$ which always is a record).

We say that, $Y_{n}=o_{p}\left(a_{n}\right)$ if $a_{n}$ is a positive number and $Y_{n}$ is a random variable such that $Y_{n} / a_{n} \stackrel{p}{\rightarrow} 0$ as $n \rightarrow \infty$.

We say that, $Y_{n}=\mathcal{O}_{L^{p}}\left(a_{n}\right)$ if $a_{n}$ is a positive number and $Y_{n}$ is a random variable such that $\left(\mathbf{E}\left(Y_{n}{ }^{p}\right)\right)^{\frac{1}{p}} \leq C a_{n}$ for some constant $C$ as $n \rightarrow \infty$.

We sometimes use the notation $m=2 \ln n$.

For simplicity in the proofs below we write $\ln n_{i}$ when we mean $\max \left\{1, \ln n_{i}\right\}$.

In the sequel we write $T$ instead of $T_{n}$. For a vertex $v \in T$, we let $T_{v}$ be the subtree of $T$ rooted at $v$, and let $n_{v}$ denote the size (number of nodes) of $T_{v}$. We can without loss of generality assume that the labels $\lambda_{v}$ have an exponential distribution $\operatorname{Exp}(1)$. As mentioned above this does not affect the distribution of $X_{n}$. We define $\varphi\left(T_{v}\right):=\mathbf{E}\left(X\left(T_{v}\right) \mid T_{v}\right)$.

Denote by $h(v)$ the height of $v$ (distance to the root). Let $\Lambda_{v_{i}}$ be the minimum of $\lambda_{v}$ along the path $P\left(v_{i}\right)=\sigma, \ldots, v_{i}$, from the root $\sigma$ of $T$, to $v_{i}, 1 \leq i \leq 2^{L}$, where $v_{i}$ are the vertices at height $L=\lfloor\beta \lg \ln n\rfloor$ for some constant $\beta$. (Below we choose $\beta \in\left(\frac{1}{\lg 3-1}, 2\right)$.) Thus, the definition of $\Lambda_{v_{i}}$ and the assumption $\lambda_{v} \in \operatorname{Exp}(1)$ give $\Lambda_{v_{i}} \in \operatorname{Exp}\left(\frac{1}{L+1}\right)$. For simplicity we write $T_{i}:=T_{v_{i}}, n_{i}:=n_{v_{i}}$ and $\Lambda_{i}:=\Lambda_{v_{i}}$. We denote $h_{i}(v):=h(v)-L$ (that is the height in the subtree $T_{i}$ of a vertex $\left.v \in T_{i}\right)$.

Define $\varphi\left(T_{i}, \Lambda_{i}\right):=\mathbf{E}\left(X\left(T_{i}\right)_{\Lambda_{i}} \mid T_{i}, \Lambda_{i}\right)$ as the conditional expected value given the tree $T_{i}$ and $\Lambda_{i}$. (We can think of $X\left(T_{i}\right)_{\Lambda_{i}}$ as $X\left(T_{i}\right)-1$ conditioned on the root label $\lambda_{v_{i}}=\Lambda_{i}$.) Define $\psi\left(T_{i}, \Lambda_{i}\right):=\operatorname{Var}\left(X\left(T_{i}\right)_{\Lambda_{i}} \mid T_{i}, \Lambda_{i}\right)$ for vertices $v_{i}, 1 \leq i \leq 2^{L}$, as the conditional variance given the tree $T_{i}$ and $\Lambda_{i}$. The conditional expected value of a random variable $Z$ given the subtree size $n_{i}$ of $T_{i}$ is denoted by $\mathbf{E}_{n_{i}}(Z):=\mathbf{E}\left(Z \mid n_{i}\right)$ and similarly we let $\operatorname{Var}_{n_{i}}(Z):=\operatorname{Var}\left(Z \mid n_{i}\right)$.

For the later part of the proof of Theorem 1.1, we also define $\mathscr{G}_{j}$ for $j=1,2 \ldots$ as the $\sigma$-field generated by $\left\{U_{r, v}, r=1,2 \ldots, j\right\}$ for all vertices $v$ with $h(v)=j$, or equivalently this is the the $\sigma$-field generated by $\left\{\mathcal{U}_{v}, h(v)<j\right\}$. 
Lemma 2.1. For all subtrees $T_{i}$ rooted at $v_{i}$ with $h\left(v_{i}\right)=L$ conditioning on (the subtree size) $n_{i}$ gives

$$
\begin{aligned}
\varphi\left(T_{i}, \Lambda_{i}\right)=\frac{n_{i}}{2 \ln n_{i}}\left(1-e^{-\left(2 \ln n_{i}\right) \Lambda_{i}}\right) & +\frac{n_{i}}{4 \ln ^{2} n_{i}} \\
& -\frac{\Upsilon\left(T_{i}\right)-2 n_{i} \ln n_{i}}{4 \ln ^{2} n_{i}}+\mathcal{O}_{L^{1}}\left(\frac{n_{i}}{\ln ^{2.2} n_{i}}\right) .
\end{aligned}
$$

where $\Upsilon\left(T_{i}\right)$ is the total path length of the tree $T_{i}$.

This conditional expected value appears in Lemma 2.3 below. This is the corresponding result to [11, Lemma 2.1 and Lemma 2.2].

Proof of Lemma 2.1. Let, for each vertex $v \in T_{i}, I_{v}$ be the indicator that $\lambda_{v}$ is the minimum value (the smallest of all the values attached to the nodes in the unique path from the given vertex $v$ to the root $v_{i}$ ) given $T_{i}$ and $\Lambda_{i}$. We get that $\varphi\left(T_{i}, \Lambda_{i}\right)=\sum_{v \neq v_{i}} \mathbf{E} I_{v}$. If $h_{i}(v)=j$, let $v_{i}, v_{i 1}, \ldots, v_{i j}=v$ be the vertices in the path from the root $v_{i}$ to $v$. Then $I_{v}=1$ if and only if $\lambda_{v_{i j}}<\Lambda_{i}$ and $\lambda_{v_{i k}}>\lambda_{v_{i j}}$ for $k \in\{1, \ldots, j-1\}$. Since the $\lambda_{v}$ 's (for all vertices $v$ in $T_{i}$ ) are independent $\operatorname{Exp}(1)$ random variables we have,

$$
\mathbf{E}\left(I_{v}\right)=\int_{0}^{\Lambda_{i}} \prod_{k=1}^{j-1} \mathbf{P}\left(\lambda_{v_{i} k}>x\right) e^{-x} d x=\int_{0}^{\Lambda_{i}} e^{-j x} d x=\frac{1-e^{-j \Lambda_{i}}}{j} .
$$

Thus,

$$
\varphi\left(T_{i}, \Lambda_{i}\right)=\sum_{v \neq v_{i}} \frac{1-e^{-h_{i}(v) \Lambda_{i}}}{h_{i}(v)} .
$$

We remember from Subsection 1.2.1 above that the binary search tree of size $n$ has most nodes at height $2 \ln n+\mathcal{O}(\sqrt{\ln n})$, see $[1,5,4]$.

Roughly, the idea of the proof is that we first find the Taylor expansion of $\sum_{v \neq v_{i}} \frac{1}{h_{i}(v)}$ about $2 \ln n_{i}$ up to the error term $\mathcal{O}_{L^{1}}\left(\frac{n_{i}}{\ln ^{2.2} n_{i}}\right)$, then using that many nodes are close to $2 \ln n_{i}$ we show that if we multiply this expansion by $\left(1-e^{-\left(2 \ln n_{i}\right) \Lambda_{i}}\right)$, from (8) we get (6).

A quite sharp profile estimate of the binary search tree one gets from [1, p.1043-1044]. Uniformly for all $k \in[(1+\epsilon) \ln n,(2+\sqrt{2}-\epsilon) \ln n]$, the number of nodes at height $k$ in the binary search tree of size $n$ (this number is denoted by $V_{k}(n)$ in [1]) is

$$
\frac{n}{\sqrt{4 \pi \ln n}} \cdot e^{-\frac{(k-2 \ln n)^{2}}{4 \ln n}}+\mathcal{O}_{L^{1}}\left(\frac{n}{\ln n}\right) .
$$


(The profile result in (9) is obtained from a generating function and asymptotics for Stirling numbers of the first kind.)

We say a vertex $v \in T_{i}$ is "good" if

$$
2 \ln n_{i}-\ln ^{0.6} n_{i} \leq h_{i}(v) \leq 2 \ln n_{i}+\ln ^{0.6} n_{i}
$$

and 'bad" otherwise. As was mentioned before "most" nodes are in the strip of the good vertices. One deduces easily from [1, equation(3)] (see also [5, Theorem 1] where equation (9) above is mentioned as a particular case of a more general result of the expected profile) that the number of "bad" nodes in $T_{i}$ is bounded by $\mathcal{O}_{L^{1}}\left(n_{i}^{1-\epsilon}\right)$ for some $\epsilon>0$. Thus, since the number of bad nodes is bounded by $\mathcal{O}_{L^{1}}\left(\frac{n_{i}}{\ln ^{2.2} n_{i}}\right)$, they can be ignored.

If we expand $\frac{1}{h_{i}(v)}$ for arbitrary good $v \in T_{i}$,

$\frac{1}{h_{i}(v)}=\frac{1}{2 \ln n_{i}}-\frac{h_{i}(v)-2 \ln n_{i}}{4 \ln ^{2} n_{i}}+\frac{\left(h_{i}(v)-2 \ln n_{i}\right)^{2}}{8 \ln ^{3} n_{i}}+\mathcal{O}\left(\frac{\left|\left(h_{i}(v)-2 \ln n_{i}\right)^{3}\right|}{\ln ^{4} n_{i}}\right)$.

This implies that the total sum is, by summing over all nodes,

$$
\sum_{v \neq v_{i}} \frac{1}{h_{i}(v)}=\frac{n_{i}}{2 \ln n_{i}}-\frac{\Upsilon\left(T_{i}\right)-2 n_{i} \ln n_{i}}{4 \ln ^{2} n_{i}}+\frac{\sum_{v}\left(h_{i}(v)-2 \ln n_{i}\right)^{2}}{8 \ln ^{3} n_{i}}+\mathcal{O}_{L^{1}}\left(\frac{n_{i}}{\ln ^{2.2} n_{i}}\right) .
$$

For the third term in the expansion in (11) we use the profile estimate (9). We see that the error term $\mathcal{O}_{L^{1}}\left(\frac{n}{\ln n}\right)$ in the profile estimate in (9) is good enough in this term. Since the distance for the good vertices to $2 \ln n_{i}$ is less than $\ln ^{0.6} n_{i}$ and there are at most $2 \cdot \ln ^{0.6} n_{i}$ levels we see that the error term in (9) could not contribute to the third term in (11) more than

$$
\mathcal{O}_{L^{1}}\left(\frac{n_{i}}{\ln n_{i}}\right) \cdot \frac{\left(\ln ^{0.6} n_{i}\right)^{2} \cdot 2 \ln ^{0.6} n_{i}}{8 \ln ^{3} n_{i}}=\mathcal{O}_{L^{1}}\left(\frac{n_{i}}{\ln ^{2.2} n_{i}}\right) .
$$

Hence, the third term in the Taylor estimate in (11) is

$$
\sum_{-\infty}^{\infty}\left(k-2 \ln n_{i}\right)^{2} e^{\frac{\left(k-2 \ln n_{i}\right)^{2}}{4 \ln n_{i}}} \cdot \frac{n_{i} / \sqrt{4 \pi \ln n_{i}}}{8 \ln ^{3} n_{i}}+\mathcal{O}_{L^{1}}\left(\frac{n_{i}}{\ln ^{2.2} n_{i}}\right) .
$$

Note that we could take the sum over all $k$ in (12) since the sums over the tails for $k \leq 2 \ln n_{i}-\ln ^{0.6} n_{i}$ and $k \geq 2 \ln n_{i}+\ln ^{0.6} n_{i}$ in (12) are dominated by the error term $\mathcal{O}_{L^{1}}\left(\frac{n_{i}}{\ln ^{2.2} n_{i}}\right)$. 
To calculate the sum $\sum_{-\infty}^{\infty}\left(k-2 \ln n_{i}\right)^{2} e^{\frac{\left(k-2 \ln n_{i}\right)^{2}}{4 \ln n_{i}}}$ we use integral approximation (with an error term that is easily calculated to be bounded by $\mathcal{O}\left(\frac{n_{i}}{\ln ^{2.2} n_{i}}\right)$ ) and get

$$
\int_{-\infty}^{\infty}\left(x-2 \ln n_{i}\right)^{2} e^{\frac{\left(x-2 \ln n_{i}\right)^{2}}{4 \ln n_{i}}} d x=2 \sqrt{4 \pi} \ln n_{i} \sqrt{\ln n_{i}} .
$$

Thus, (11) implies that

$$
\sum_{v \neq v_{i}} \frac{1}{h_{i}(v)}=\frac{n_{i}}{2 \ln n_{i}}-\frac{\Upsilon\left(T_{i}\right)-2 n_{i} \ln n_{i}}{4 \ln ^{2} n_{i}}+\frac{n_{i}}{4 \ln ^{2} n_{i}}+\mathcal{O}_{L^{1}}\left(\frac{n_{i}}{\ln ^{2.2} n_{i}}\right) .
$$

Remark 2.1. We cannot use the profile approximation in (9) to calculate the second term in (11) since the error term $\mathcal{O}_{L^{1}}\left(\frac{n_{i}}{\ln n_{i}}\right)$ is too large. The total path length random variable $\Upsilon\left(T_{i}\right)$ (given $n_{i}$ ) has a variance of order $\mathcal{O}\left(n_{i}^{2}\right)$, see Subsection 1.2.2 above. Thus, the error estimate one gets is not small enough.

Now we prove that

$$
\sum_{v \neq v_{i}} \frac{1}{h_{i}(v)}\left(e^{-h_{i}(v) \Lambda_{i}}-e^{-2 \Lambda_{i} \ln n_{i}}\right)=\mathcal{O}_{L^{1}}\left(\frac{n_{i}}{\ln ^{2.2} n_{i}}\right),
$$

which by (8) obviously implies that

$$
\varphi\left(T_{i}, \Lambda_{i}\right)=(13) \cdot\left(1-e^{-\left(2 \ln n_{i}\right) \Lambda_{i}}\right)+\mathcal{O}_{L^{1}}\left(\frac{n_{i}}{\ln ^{2.2} n_{i}}\right) .
$$

For simpler calculations below we consider the term $e^{-\Lambda_{i}\left\lfloor 2 \ln n_{i}\right\rfloor}$ instead of $e^{-\left(2 \ln n_{i}\right) \Lambda_{i}}$ in (14) and (15). That one can do this follows from that multiplying (13) by $e^{-\Lambda_{i}\left\lfloor 2 \ln n_{i}\right\rfloor}$ gives the same expression up to the error term $\mathcal{O}_{L^{1}}\left(\frac{n_{i}}{\ln ^{2.2} n_{i}}\right)$ as multiplying by $e^{-2 \Lambda_{i} \ln n_{i}}$.

For $j>0$,

$$
e^{\left(-\left\lfloor 2 \ln n_{i}\right\rfloor+j\right) \Lambda_{i}}=e^{-\Lambda_{i}\left\lfloor 2 \ln n_{i}\right\rfloor}+e^{\left(-\left\lfloor 2 \ln n_{i}\right\rfloor+j\right) \Lambda_{i}} \cdot\left(1-e^{-j \Lambda_{i}}\right)
$$

and

$$
e^{\left(-\left\lfloor 2 \ln n_{i}\right\rfloor-j\right) \Lambda_{i}}=e^{-\Lambda_{i}\left\lfloor 2 \ln n_{i}\right\rfloor}+e^{\left(-\left\lfloor 2 \ln n_{i}\right\rfloor+j\right) \Lambda_{i}} \cdot\left(e^{-2 j \Lambda_{i}}-e^{-j \Lambda_{i}}\right) .
$$


We use the profile estimate in (9). In particular we need to show that

$$
\begin{aligned}
\sum_{j=1}^{\ln ^{0.6} n_{i}}\left(\left(\frac{n_{i}}{\sqrt{4 \pi \ln n_{i}}} e^{\frac{-\left(j+\left\{2 \ln n_{i}\right\}\right)^{2}}{4 \ln n_{i}}}+\mathcal{O}_{L^{1}}\left(\frac{n_{i}}{\ln n_{i}}\right)\right)\right. \\
\left.\cdot e^{\left(-\left\lfloor 2 \ln n_{i}\right\rfloor+j\right) \Lambda_{i}}\left(1-e^{-j \Lambda_{i}}\right) \frac{1}{\left\lfloor 2 \ln n_{i}\right\rfloor-j}\right)=\mathcal{O}_{L^{1}}\left(\frac{n_{i}}{\ln ^{2.2} n_{i}}\right)
\end{aligned}
$$

and

$$
\begin{aligned}
\sum_{j=1}^{\ln ^{0.6} n_{i}} & \left(\left(\frac{n_{i}}{\sqrt{4 \pi \ln n_{i}}} \cdot e^{\frac{-\left(j-\left\{2 \ln n_{i}\right\}\right)^{2}}{4 \ln n_{i}}}+\mathcal{O}_{L^{1}}\left(\frac{n_{i}}{\ln n_{i}}\right)\right)\right. \\
& \left.\cdot e^{\left(-\left\lfloor 2 \ln n_{i}\right\rfloor+j\right) \Lambda_{i}}\left(e^{-2 j \Lambda_{i}}-e^{-j \Lambda_{i}}\right) \frac{1}{\left\lfloor 2 \ln n_{i}\right\rfloor+j}\right)=\mathcal{O}_{L^{1}}\left(\frac{n_{i}}{\ln ^{2.2} n_{i}}\right)
\end{aligned}
$$

where $\left\{2 \ln n_{i}\right\}$ is the fractional part of $2 \ln n_{i}$.

We show that $(16)+(17)=\mathcal{O}_{L^{1}}\left(\frac{n_{i}}{\ln ^{2.2} n_{i}}\right)$ is true. Taylor expansion gives

$$
(16)=\sum_{j=1}^{\ln ^{0.6} n_{i}}\left(\frac{n_{i}}{\sqrt{4 \pi \ln n_{i}}} e^{\frac{-\left(j+\left\{2 \ln n_{i}\right\}\right)^{2}}{4 \ln n_{i}}}+\mathcal{O}_{L^{1}}\left(\frac{n_{i}}{\ln n_{i}}\right)\right) e^{\left(-\left\lfloor 2 \ln n_{i}\right\rfloor+j\right) \Lambda_{i}} \mathcal{O}\left(\frac{j \Lambda_{i}}{\ln n_{i}}\right) .
$$

We calculate

$$
\int_{j=0}^{\infty} j e^{\frac{-\left(j+\left\{2 \ln n_{i}\right\}\right)^{2}}{4 \ln n_{i}}} d j=\mathcal{O}\left(\ln n_{i}\right)
$$

Since $\Lambda_{i}$ is an $\operatorname{Exp}\left(\frac{1}{L+1}\right)$ random variable, we have that, for all $j \leq \ln ^{0.6} n_{i}$

$$
\begin{aligned}
\mathbf{E} \Lambda_{i} e^{\left(-\left\lfloor 2 \ln n_{i}\right\rfloor+j\right) \Lambda_{i}} & =\int_{0}^{\infty}(L+1) y e^{\left(-\left\lfloor 2 \ln n_{i}\right\rfloor+j\right) y} e^{-y(L+1)} d y \\
& =\frac{L+1}{\left(\left\lfloor 2 \ln n_{i}\right\rfloor-j+L+1\right)^{2}} .
\end{aligned}
$$

Thus,

$$
\sum_{j=1}^{\ln ^{0.6} n_{i}} \frac{n_{i}}{\sqrt{4 \pi \ln n_{i}}} e^{\frac{-\left(j+\left\{2 \ln n_{i}\right\}\right)^{2}}{4 \ln n_{i}}} e^{\left(-\left\lfloor 2 \ln n_{i}\right\rfloor+j\right) \Lambda_{i}} \mathcal{O}\left(\frac{j \Lambda_{i}}{\ln n_{i}}\right)=\mathcal{O}_{L^{1}}\left(\frac{n_{i}}{\ln ^{2.2} n_{i}}\right) .
$$

We easily check that also the error term in the profile estimate can be ignored so that

$$
\sum_{j=1}^{\ln ^{0.6} n_{i}} \mathcal{O}_{L^{1}}\left(\frac{n_{i}}{\ln n_{i}}\right) e^{\left(-\left\lfloor 2 \ln n_{i}\right\rfloor+j\right) \Lambda_{i}} \mathcal{O}\left(\frac{j \Lambda_{i}}{\ln n_{i}}\right)=\mathcal{O}_{L^{1}}\left(\frac{n_{i}}{\ln ^{2.2} n_{i}}\right) .
$$


Thus, $(16)=\mathcal{O}_{L^{1}}\left(\frac{n_{i}}{\ln ^{2} n_{i}}\right)$. By similar calculations one shows that also (17) $=\mathcal{O}_{L^{1}}\left(\frac{n_{i}}{\ln ^{2.2} n_{i}}\right)$. Thus, it follows that (15) is satisfied.

We have $e^{-\left(2 \ln n_{i}\right) \Lambda_{i}}=\mathcal{O}_{L^{1}}\left(\frac{L}{\ln n_{i}}\right)$, hence $e^{-\left(2 \ln n_{i}\right) \Lambda_{i}} \cdot \mathcal{O}\left(\frac{n_{i}}{\ln ^{2} n_{i}}\right)=\mathcal{O}_{L^{1}}\left(\frac{n_{i}}{\ln ^{2.2} n_{i}}\right)$.

Since also $\mathbf{E}\left(e^{-\left(2 \ln n_{i}\right) \Lambda_{i}}\right)^{2}=\mathcal{O}\left(\frac{L}{\ln n_{i}}\right)$ and (see Section 1.2.2)

$$
\mathbf{E}_{n_{i}}\left(\frac{\Upsilon\left(T_{i}\right)-2 n_{i} \ln n_{i}}{4 \ln ^{2} n_{i}}\right)^{2}=\mathcal{O}\left(\frac{n_{i}^{2}}{\ln ^{4} n_{i}}\right)
$$

one gets

$$
\frac{\left(\Upsilon\left(T_{i}\right)-2 n_{i} \ln n_{i}\right) e^{-\left(2 \ln n_{i}\right) \Lambda_{i}}}{4 \ln ^{2} n_{i}}=\mathcal{O}_{L^{2}}\left(\frac{n_{i}}{\ln ^{2.2} n_{i}}\right)=\mathcal{O}_{L^{1}}\left(\frac{n_{i}}{\ln ^{2.2} n_{i}}\right)
$$

(where the last equality is obtained by using the well-known Lyapounov inequality e.g. [9, p.129]) and Lemma 2.1 follows.

Lemma 2.2. For all vertices $v_{i}$ with $h\left(v_{i}\right)=L$, conditioned on $n_{i}$,

$$
\mathbf{E}_{n_{i}}\left(\psi\left(T_{i}, \Lambda_{i}\right)\right)=\mathcal{O}\left(\frac{n_{i}^{2}}{\ln ^{3} n_{i}}\right) .
$$

This is the corresponding result to [11, Lemma 2.1 (2nd part)]. This estimate is used in Lemma 2.3 below.

Proof of Lemma 2.2. We condition on the binary search tree $T_{i}$ and $\Lambda_{i}$ and for all vertices $v \in T_{i}$ we let $I_{v}$ be the same indicator as in the proof of Lemma 2.1 above.

Suppose that $v$ and $w$ are two vertices in $T_{i}$ at height $h_{i}(v)=j, h_{i}(w)=k$ with their last common ancestor at height $h_{i}(u)=h$. Suppose first that $h<j, h<k$. (In fact we show below that other choices of vertices can be ignored.) Let $\left\{v_{i}, u_{1}, \ldots, u_{h}=u\right\}$ be the vertices in the path from $v_{i}$ to $u$ and let $Z=\min \left\{\lambda_{u_{s}}: 1 \leq s \leq h\right\}$. Conditioned on $Z, I_{v}$ and $I_{w}$ are independent. Let $Z \wedge \Lambda_{i}$ denote the minimum of $Z$ and $\Lambda_{i}$. Since $v$ has height $j-h$ above $u,(7)$ yields

$$
\mathbf{E}\left(I_{v} \mid Z\right)=\frac{1-e^{-(j-h)\left(Z \wedge \Lambda_{i}\right)}}{j-h}
$$


and similarly for $I_{w}$. See [11, p.244]. As in [11, equation (6) p.245]

$$
\begin{gathered}
\mathbf{E}\left(I_{v} I_{w}\right)=\frac{1}{j-h} \frac{1}{k-h}\left(1-e^{-h \Lambda_{i}}-\frac{h}{j}\left(1-e^{-j \Lambda_{i}}\right)-\frac{h}{k}\left(1-e^{-k \Lambda_{i}}\right)\right. \\
\left.+\frac{h}{j+k-h}\left(1-e^{-(j+k-h) \Lambda_{i}}\right)+e^{-h \Lambda_{i}}-e^{-j \Lambda_{i}}-e^{-k \Lambda_{i}}+e^{-(j+k-h) \Lambda_{i}}\right) .
\end{gathered}
$$

The covariance of $I_{v}$ and $I_{w}$ is:

$$
\operatorname{Cov}\left(I_{v}, I_{w}\right)=\mathbf{E}\left(I_{v} I_{w}\right)-\mathbf{E}\left(I_{v}\right) \mathbf{E}\left(I_{w}\right)
$$

Remark 2.2. The fewer common vertices in $v^{\prime} s$ and $w^{\prime} s$ paths to the root the smaller covariance of $I_{v}$ and $I_{w}$. Consequently, to minimize the covariance we want $h_{i}(v)=j$ and $h_{i}(w)=k$ to be large and $h_{i}(u)=h$ to be small.

We say that a pair $(v, w)$ is "good" if $j$ and $k$ satisfy

$$
2 \ln n_{i}-\ln ^{0.6} n_{i} \leq j, k \leq 2 \ln n_{i}+\ln ^{0.6} n_{i}
$$

and $h \leq \alpha \ln n_{i}$ for some $\alpha$ that we choose small enough, and "bad" otherwise. If $\alpha$ is chosen to be small it is obvious that the covariance estimate for the good pairs of vertices in the binary search tree is not worse than the order of the estimate for the complete binary tree in [11]. Thus, (from [11, equation (7) p.245] ) by (7) and (21) above for a good pair

$$
\begin{aligned}
\operatorname{Cov}\left(I_{v}, I_{w}\right) & =\mathbf{E}\left(I_{v} I_{w}\right)-\mathbf{E}\left(I_{v}\right) \mathbf{E}\left(I_{w}\right)=\frac{1}{j k} e^{-(j+k-h) \Lambda_{i}}\left(1-e^{-h \Lambda_{i}}\right)+\mathcal{O}\left(\frac{h}{\ln ^{3} n_{i}}\right) \\
& =\mathcal{O}\left(\frac{1}{\ln ^{2} n_{i}} e^{-(j+k-h) \Lambda_{i}} h \Lambda_{i}\right)+\mathcal{O}\left(\frac{h}{\ln ^{3} n_{i}}\right) \\
& =\mathcal{O}_{L^{1}}\left(\frac{h L}{\ln ^{4} n_{i}}\right)+\mathcal{O}\left(\frac{h}{\ln ^{3} n_{i}}\right)=\mathcal{O}_{L^{1}}\left(\frac{h}{\ln ^{3} n_{i}}\right)
\end{aligned}
$$

where we in the fourth equality use $e^{-(j+k-h) \Lambda_{i}} h \Lambda_{i}=\mathcal{O}_{L^{1}}\left(\frac{h L}{\ln ^{2} n_{i}}\right)$ (that is obtained by similar calculations as in (19) above).

Using the result of the profile estimate we discussed in the proof of Lemma 2.1 that there are $\mathcal{O}_{L^{1}}\left(n_{i}^{1-\epsilon}\right)$ nodes that are not in

$$
2 \ln n_{i}-\ln ^{0.6} n_{i} \leq h(v) \leq 2 \ln n_{i}+\ln ^{0.6} n_{i},
$$

it follows that if $v$ and $w$ are not both in this strip there are $\mathcal{O}_{L^{1}}\left(n_{i}^{2-\epsilon}\right)$ ways to choose the pair $v, w$ for some $\epsilon>0$. 
Let $n_{h k}, 1 \leq k \leq 2^{h}$, be the subtree sizes rooted at height $h$ (they are random numbers) in $T_{i}$. For a given height $h$ there are at most $\sum_{k=1}^{2^{h}} n_{h k}^{2}$ choices of $v$ and $w$. Now we use the subtree method above, see Subsection 1.2 .3 , to calculate the expected value of $n_{h k}^{2}, 1 \leq k \leq 2^{h}$. Note that they all have the same expected value since they are on the same level (height) from the root $v_{i}$. It is easy to see that

$$
\mathbf{E}_{n_{i}}\left(n_{h k}\right) \leq\left(\prod_{r=1}^{h} \mathbf{E} U_{r}\right) n_{i}=\frac{n_{i}}{2^{h}}, \quad \mathbf{E}_{n_{i}}\left(n_{h k}^{2}\right) \leq\left(\prod_{r=1}^{h} \mathbf{E} U_{r}^{2}\right) n_{i}^{2}=\frac{n_{i}^{2}}{3^{h}}
$$

since the $U_{r}$ 's are independent uniform random variables. Consequently, the expected number of pairs of vertices with $h>\alpha \ln n_{i}$ is

$$
\mathbf{E}_{n_{i}}\left(\sum_{h>\alpha \ln } \sum_{n_{i}}^{2^{i}} n_{h=1}^{2}\right)=\mathcal{O}\left(\sum_{h>\alpha \ln n_{i}} \frac{n_{i}^{2} \cdot 2^{h}}{3^{h}}\right)=\mathcal{O}\left(\frac{n_{i}^{2}}{\ln ^{3} n_{i}}\right) .
$$

Thus, the number of "bad" pairs of vertices is $\mathcal{O}_{L^{1}}\left(\frac{n_{i}^{2}}{\ln ^{3} n_{i}}\right)$.

Now for the random binary search tree $T_{i}$ on $n_{i}$ vertices

$$
\psi\left(T_{i}, \Lambda_{i}\right)=\sum_{v, w \in T_{i}} \operatorname{Cov}\left(I_{v}, I_{w}\right)
$$

( $\psi$ is a random variable depending on the random tree $T_{i}$ and $\Lambda_{i}$ ).

Since we have the the obvious upper bound $\operatorname{Cov}\left(I_{v}, I_{w}\right) \leq 1$, the bad pairs can be ignored. Thus,

$$
\mathbf{E}_{n_{i}}\left(\psi\left(T_{i}, \Lambda_{i}\right)\right)=\mathbf{E}_{n_{i}}\left(\sum_{\operatorname{good} v, w) \in T_{i}} \operatorname{Cov}\left(I_{v}, I_{w}\right)\right)+\mathcal{O}\left(\frac{n_{i}^{2}}{\ln ^{3} n_{i}}\right) .
$$

Using $\mathbf{E}_{n_{i}}\left(n_{h k}^{2}\right) \leq \frac{n_{i}^{2}}{3^{h}}$ (see equation (23)) and equation (22) give

$$
\begin{aligned}
\mathbf{E}_{n_{i}}\left(\psi\left(T_{i}, \Lambda_{i}\right)\right) & \left.=\mathcal{O}_{L^{1}}\left(\mathbf{E}_{n_{i}} \sum_{\operatorname{good}(v, w) \in T_{i}} \frac{h}{\ln ^{3} n_{i}}\right)\right)+\mathcal{O}\left(\frac{n_{i}^{2}}{\ln ^{3} n_{i}}\right) \\
& =\mathcal{O}\left(\sum_{h} \frac{n_{i}^{2} \cdot 2^{h} \cdot h}{3^{h} \ln ^{3} n_{i}}\right)+\mathcal{O}\left(\frac{n_{i}^{2}}{\ln ^{3} n_{i}}\right)=\mathcal{O}\left(\frac{n_{i}^{2}}{\ln ^{3} n_{i}}\right) .
\end{aligned}
$$

The estimate in (25) is used in the proof of the following result. 
Lemma 2.3. For a binary search tree on $n$ vertices let $v_{i}, 1 \leq i \leq 2^{L}$ be the vertices at height $L=\lfloor\beta \lg \ln n\rfloor$ for $\beta>\frac{1}{\lg 3-1}$. Then

$$
X\left(T_{n}\right)=\sum_{i=1}^{2^{L}} \varphi\left(T_{i}, \Lambda_{i}\right)+o_{p}\left(\frac{n}{\ln ^{2} n}\right)
$$

This is the corresponding result to [11, Lemma 2.3 equation (10)].

Proof of Lemma 2.3. We write the number of records as $\left\{V^{*}+V_{1}+\ldots+V_{2^{L}}\right\}$ where $V^{*}$ is the number of records with height $\leq L$ and $V_{i}$ is the number of records in the subtree $T_{i}$ that is rooted at vertex $v_{i}$ at height $L$ except for the root $v_{i}$.

Let $\mathscr{F}_{L}$ be the $\sigma$-field generated by $\left\{\lambda_{v}: h(v) \leq L\right\}$ and $\mathscr{F}_{L}^{*}$ the $\sigma$ - field generated by $T_{n}$ and $\mathscr{F}_{L}$. Conditioned on $\mathscr{F}_{L}^{*}, V^{*}$ and $\Lambda_{i}$ become fixed and $\mathbf{E}\left(V_{i} \mid \mathscr{F}_{L}^{*}\right)=\varphi\left(T_{i}, \Lambda_{i}\right)$.

Given $\mathscr{F}_{L}^{*}$, the variables $V_{i}$ and $V_{j}, \quad i \neq j$ are independent and thus

$$
\begin{aligned}
& \mathbf{E}\left(\left(X_{n}-V^{*}-\sum_{i=1}^{2^{L}} \varphi\left(T_{i}, \Lambda_{i}\right)\right)^{2} \mid \mathscr{F}_{L}^{*}\right)=\mathbf{E}\left(\left(\sum_{i=1}^{2^{L}} V_{i}-\varphi\left(T_{i}, \Lambda_{i}\right)\right)^{2} \mid \mathscr{F}_{L}^{*}\right) \\
& =\sum_{i=1}^{2^{L}} \mathbf{E}\left(\left(V_{i}-\varphi\left(T_{i}, \Lambda_{i}\right)\right)^{2} \mid \mathscr{F}_{L}^{*}\right)=\sum_{i=1}^{2^{L}} \psi\left(T_{i}, \Lambda_{i}\right) .
\end{aligned}
$$

Now taking the expectation of (27) yields

$$
\mathbf{E}\left(\left(X\left(T_{n}\right)-V^{*}-\sum_{i=1}^{2^{L}} \varphi\left(T_{i}, \Lambda_{i}\right)\right)^{2}\right)=\mathbf{E}\left(\sum_{i=1}^{2^{L}} \psi\left(T_{i}, \Lambda_{i}\right)\right)=\sum_{i=1}^{2^{L}} \mathbf{E} \psi\left(T_{i}, \Lambda_{i}\right)
$$

By using Lemma 2.2 and observing the obvious fact that one can ignore those $n_{i}<\frac{n}{2^{k L}}$ for $k$ large enough (there are $2^{L}$ vertices at height $L$ ) give that

$$
\sum_{i=1}^{2^{L}} \mathbf{E}_{n_{i}}\left(\psi\left(T_{i}, \Lambda_{i}\right)\right)=\mathcal{O}\left(\sum_{i=1}^{2^{L}} \frac{n_{i}^{2}}{\ln ^{3} n_{i}}\right)=\mathcal{O}\left(\sum_{i=1}^{2^{L}} \frac{n_{i}^{2}}{\ln ^{3} n}\right) .
$$

The expected value of (29) is (28) and thus from equation (23) above

$$
(28)=\mathcal{O}\left(\frac{2^{L} n^{2}}{3^{L} \ln ^{3} n}\right) .
$$


If the constant $\beta>\frac{1}{\lg 3-1}$ in $L$ then

$$
(30)=o\left(\frac{n^{2}}{\ln ^{4} n}\right) .
$$

This implies by using the well-known Markov inequality (e.g. [9, p. 120]) that

$$
X\left(T_{n}\right)-V^{*}-\sum_{i=1}^{2^{L}} \varphi\left(T_{i}, \Lambda_{i}\right)=o_{p}\left(\frac{n}{\ln ^{2} n}\right) .
$$

The result follows because also

$$
V^{*}<2^{L+1}=\mathcal{O}\left(\ln ^{\beta} n\right)=o\left(\frac{n}{\ln ^{2} n}\right) .
$$

Since one can ignore those $n_{i}<\frac{n}{2^{k L}}$ for $k$ large enough by Taylor expansion

$$
\sum_{i=1}^{2^{L}} \frac{n_{i}}{\ln ^{2.2} n_{i}}=\frac{n}{\ln ^{2.2} n}+\mathcal{O}\left(\frac{n \ln \ln n}{\ln ^{3} n}\right)
$$

Using this and $\mathcal{O}_{L^{1}}\left(\frac{n}{\ln ^{2.2} n}\right)=o_{p}\left(\frac{n}{\ln ^{2} n}\right)$ (from the Markov inequality), applying Lemma 2.1 and Lemma 2.3 yields for $\beta>\frac{1}{\lg 3-1}$

$$
X\left(T_{n}\right)=\sum_{i=1}^{2^{L}}\left(-\frac{n_{i} e^{-\left(2 \ln n_{i}\right) \Lambda_{i}}}{2 \ln n_{i}}+\frac{n_{i}}{4 \ln ^{2} n_{i}}+\frac{n_{i}}{\ln n_{i}}-\frac{\Upsilon\left(T_{i}\right)}{4 \ln ^{2} n_{i}}\right)+o_{p}\left(\frac{n}{\ln ^{2} n}\right) .
$$

Similarly as in (33)

$$
\sum_{i=1}^{2^{L}} \frac{n_{i}}{4 \ln ^{2} n_{i}}=\frac{n}{4 \ln ^{2} n}+\mathcal{O}\left(\frac{n \ln \ln n}{\ln ^{3} n}\right)
$$

Again we use that one can ignore $n_{i}<\frac{n}{2^{k L}}$ for $k$ large enough, comparing with [11, equations $(12,13)$ p.247] for $n_{i} \geq \frac{n}{2^{k L}}$

$\mathbf{E}_{n_{i}}\left(\left|e^{-\left(2 \ln n_{i}\right) \Lambda_{i}}-e^{-(2 \ln n) \Lambda_{i}}\right|\right)=\frac{L+1}{L+1+2 \ln n_{i}}-\frac{L+1}{L+1+2 \ln n}=\mathcal{O}\left(\frac{L^{2}}{\ln ^{2} n}\right)$, and it follows that 


$$
\mathbf{E}\left(\left|\frac{n_{i}}{2 \ln n_{i}} e^{-\left(2 \ln n_{i}\right) \Lambda_{i}}-\frac{n_{i}}{2 \ln n} e^{-(2 \ln n) \Lambda_{i}}\right|\right)=\mathcal{O}\left(\frac{L^{2} n}{2^{L} \ln ^{3} n}\right) .
$$

Thus, for $\beta>\frac{1}{\lg 3-1}$ with another application of the Markov inequality (e.g. [9]),

$$
X\left(T_{n}\right)=\sum_{i=1}^{2^{L}} \frac{n_{i}}{\ln n_{i}}-\sum_{i=1}^{2^{L}} \frac{\Upsilon\left(T_{i}\right)}{4 \ln ^{2} n_{i}}-\frac{1}{2 \ln n} \sum_{i=1}^{2^{L}} n_{i} e^{-(2 \ln n) \Lambda_{i}}+\frac{n}{4 \ln ^{2} n}+o_{p}\left(\frac{n}{\ln ^{2} n}\right) .
$$

Lemma 2.4. If the constant $\beta$ in $L=\lfloor\beta \lg \ln n\rfloor$ is $<2$, then

$$
\sum_{i=1}^{2^{L}} n_{i} e^{-(2 \ln n) \Lambda_{i}}=\sum_{h(v) \leq L} n_{v} e^{-(2 \ln n) \lambda_{v}}+o_{p}\left(\frac{n}{\ln n}\right)
$$

Thus, choosing $\beta \in\left(\frac{1}{\lg 3-1} 2\right)$ from (35)

$$
\begin{aligned}
X\left(T_{n}\right)=\sum_{i=1}^{2^{L}} \frac{n_{i}}{\ln n_{i}} & -\sum_{i=1}^{2^{L}} \frac{\Upsilon\left(T_{i}\right)}{4 \ln ^{2} n_{i}} \\
& -\frac{1}{2 \ln n} \sum_{h(v) \leq L} n_{v} e^{-(2 \ln n) \lambda_{v}}+\frac{n}{4 \ln ^{2} n}+o_{p}\left(\frac{n}{\ln ^{2} n}\right) .
\end{aligned}
$$

(Compare with [11, Lemma 2.4].)

Proof of Lemma 2.4. The proof follows by the same calculations as in [11, Lemma 2.4]. Choosing $a=\frac{2 \ln m}{m}$ where $m=2 \ln n$ we see as in [11, p.248] that the probability for some $i$ that at least two $\lambda_{v}, v \in P\left(v_{i}\right)$ are less than $a$ is

$$
\mathcal{O}\left(\frac{2^{L} \ln ^{4} m}{m^{2}}\right)=o(1),
$$

so that with probability tending to 1 there is at most one $\lambda_{v}$ less than $a$ in each $P\left(v_{i}\right)$. This gives that

$$
0 \leq \sum_{v \in P\left(v_{i}\right)} e^{-m \lambda_{v}}-e^{-m \Lambda_{i}} \leq L e^{-m a}=\frac{L}{m^{2}} .
$$

From (38) it is quite easy to get the result. (See [11, p. 248].) 
Remark 2.3. From (37) we see why we choose the constant $\beta$ in $L$ as less than 2 .

We recollect the definition of $\mathscr{G}_{j}$ in the beginning of Section 2 as the $\sigma$ field generated by $\left\{U_{r, v}, r=1,2 \ldots, j\right\}$ for all vertices $v$ with $h(v)=j$. In particular we get that $\mathscr{G}_{L}$ is the $\sigma$-field generated by $\left\{U_{r, v}, r=1,2 \ldots, L\right\}$ for all vertices $v$ with $h(v)=L$. Note that in particular the $\sigma$-field generated by $\left\{n_{v}, h(v) \leq L\right\}$ is a sub- $\sigma$-field of $\mathscr{G}_{L}$.

Conditioned on $\mathscr{G}_{L}$ the summands $\Upsilon\left(T_{i}\right)$ in (36) are independent thus from Subsection 1.2.2 we obtain

$$
\mathbf{E}\left(\sum_{i=1}^{2^{L}} \frac{\Upsilon\left(T_{i}\right)}{4 \ln ^{2} n_{i}} \mid \mathscr{G}_{L}\right)=\sum_{i=1}^{2^{L}} \frac{n_{i}}{2 \ln n_{i}}+\sum_{i=1}^{2^{L}} \frac{n_{i}(2 \gamma-4)}{4 \ln ^{2} n_{i}}+\sum_{i=1}^{2^{L}} \mathcal{O}\left(\frac{1}{\ln n_{i}}\right),
$$

and

$$
\operatorname{Var}\left(\sum_{i=1}^{2^{L}} \frac{\Upsilon\left(T_{i}\right)}{4 \ln ^{2} n_{i}} \mid \mathscr{G}_{L}\right)=\mathcal{O}\left(\sum_{i=1}^{2^{L}} \frac{n_{i}^{2}}{\ln ^{4} n_{i}}\right)
$$

Thus, in analogy with $(30)$ and(31), given $\mathscr{G}_{L}$

$$
\sum_{i=1}^{2^{L}} \frac{\Upsilon\left(T_{i}\right)}{4 \ln ^{2} n_{i}}=\sum_{i=1}^{2^{L}} \frac{n_{i}}{2 \ln n_{i}}+\frac{n(2 \gamma-4)}{4 \ln ^{2} n}+o_{p}\left(\frac{n}{\ln ^{2} n}\right) .
$$

For simplicity (in the calculations below) we change the notation $n_{i}, 1 \leq 2^{L}$, to $n_{v}, h(v)=L$.

Thus, from (36), choosing the constant $\beta \in\left(\frac{1}{\lg 3-1}, 2\right)$,

$$
X\left(T_{n}\right)=\sum_{h(v)=L} \frac{n_{v}}{2 \ln n_{v}}-\frac{1}{2 \ln n} \sum_{h(v) \leq L} n_{v} e^{-(2 \ln n) \lambda_{v}}+\frac{n(5-2 \gamma)}{4 \ln ^{2} n}+o_{p}\left(\frac{n}{\ln ^{2} n}\right) .
$$

As in [11], the proof of Theorem 1.1 will be completed by a classical theorem for convergence of triangular arrays to infinitely divisible distributions, see e.g. [14, Theorem 15.28]. Since the $n_{v}$ 's in the sums in (40) are not independent (although they are less dependent for vertices that are far from each other), $\left\{\xi_{v}=\frac{m n_{v}}{n} e^{-m \lambda_{v}}\right\}$ is not a triangular array. For the sake of independence we intend to condition on the $n_{v}$ 's in the sums in (40) and show that (after normalization) we get convergence in distribution to $W$ with an 
infinitely divisible distribution, which is not depending on the $n_{v}$ 's we conditioned on. That is, after normalization also $X\left(T_{n}\right)$ converges in distribution to $W$. Because of (40) and the notation of $\xi_{v}$

$$
\begin{aligned}
& \frac{4 \ln ^{2} n}{n}\left(X_{v}\left(T_{n}\right)-\frac{n}{2 \ln n}-\frac{n \ln \ln n}{2 \ln ^{2} n}\right) \\
& =-\sum_{h(v) \leq L} \xi_{v}+\frac{4 \ln ^{2} n}{n} \sum_{h(v)=L} \frac{n_{v}}{2 \ln n_{v}}-2 \ln \ln n-2 \ln n+5-2 \gamma+o_{p}(1) .
\end{aligned}
$$

The next theorem will imply Theorem 1.1 above.

Theorem 2.1. Suppose that $n \rightarrow \infty$ and choose any constant $c>0$ ( $\gamma$ is the Euler constant). Conditioning on the $\sigma$-field $\mathscr{G}_{L}$ defined above (where $\left.L=\lfloor\beta \lg \ln n\rfloor, \beta \in\left(\frac{1}{\lg 3-1}, 2\right)\right)$ the following hold:

(i) $\sup _{v} \mathbf{P}\left(\xi_{v}>x \mid \mathscr{G}_{L}\right) \rightarrow 0$ for every $x>0$,

(ii) $\sum_{h(v) \leq L} \mathbf{P}\left(\xi_{v}>x \mid \mathscr{G}_{L}\right) \stackrel{p}{\rightarrow} \nu(x, \infty)=\frac{2}{x}$ for every $x>0$,

(iii) $\sum_{h(v) \leq L} \mathbf{E}\left(\xi_{v} \mathbf{1}\left[\xi_{v} \leq c\right] \mid \mathscr{G}_{L}\right)-\frac{4 \ln ^{2} n}{n} \sum_{h(v)=L} \frac{n_{v}}{2 \ln n_{v}}$

$+2 \ln \ln n+2 \ln n-5+2 \gamma \stackrel{p}{\rightarrow}-3-2 \ln 2+2 \gamma+2 \ln c$,

(iv) $\sum_{h(v) \leq L} \operatorname{Var}\left(\xi_{v} \mathbf{1}\left[\xi_{v} \leq c\right] \mid \mathscr{G}_{L}\right) \stackrel{p}{\rightarrow} 2 c$.

Before proving this theorem we show how it will imply Theorem 1.1 above. Let $C=\frac{4 \ln ^{2} n}{n} \sum_{h(v)=L} \frac{n_{v}}{2 \ln n_{v}}-2 \ln \ln n-2 \ln n+5-2 \gamma$. We apply [14, Theorem 15.28] with $a=0$ and $b=-3-2 \ln 2+2 \gamma$ to $\sum_{h(v) \leq L} \xi_{v}+\sum_{i=1}^{n} \xi_{i}^{\prime}$ conditioned on $\mathscr{G}_{L}$ with $\xi_{i}^{\prime}=\frac{-C}{n}$ deterministic. (Note that $\frac{C}{n} \rightarrow 0$, thus $\left\{\xi_{v}\right\} \bigcup\left\{\xi_{i}^{\prime}\right\}$ is a null array.) We define $S(n):=\sum_{h(v) \leq L} \xi_{v}+\sum_{i=1}^{n} \xi_{i}^{\prime}$. We have $\frac{d \nu}{d x}=\frac{2}{x^{2}}$, hence

$$
\begin{aligned}
& \int_{0}^{c} x^{2} d \nu(x)=\int_{0}^{c} 2 d x=2 c, \\
& \int_{c}^{1} x d \nu(x)=\int_{c}^{1} \frac{2}{x} d x=-2 \ln c .
\end{aligned}
$$


The convergence in Theorem 2.1 is in probability, while [14, Theorem 15.28] requires usual convergence.

(For the complete binary tree Janson applies [14, Theorem 15.28] by using the result in [11, Lemma 2.5], in contrast to Theorem 2.1 the convergence in this lemma is in the usual rather than deterministic sense due to the fact that the $n_{v}$ 's are fixed for this tree.) However, if the convergence instead was a.s. in Theorem 2.1, then it would have been easy to see from this theorem that conditionally on $\mathscr{G}_{L}$, the conditions of [14, Theorem 15.28] are fullfilled, implying

$$
\sum_{h(v) \leq L} \xi_{v}-\frac{4 \ln ^{2} n}{n} \sum_{h(v)=L} \frac{n_{v}}{2 \ln n_{v}}+2 \ln \ln n+2 \ln n-5+2 \gamma=S(n) \stackrel{d}{\rightarrow} W
$$

conditioned on $\mathscr{G}_{L}$.

It follows from (42) that conditioning on $\mathscr{G}_{L}$ has no influence on the distributional convergence of $S(n)$ (unconditioned), since for any continuous bounded function $g: \Re \rightarrow \Re$,

$$
\mathbf{E} g\left(S(n) \mid \mathscr{G}_{L}\right)=\int g d F\left(S(n) \mid \mathscr{G}_{L}\right) \rightarrow \mathbf{E} g(W)
$$

Thus, taking expectation by dominated convergence

$$
\mathbf{E} g(S(n)) \rightarrow \mathbf{E} g(W) .
$$

This shows that $S\left(\zeta_{n}\right) \stackrel{d}{\rightarrow} W$. Compare this with [12] and note the difference that the measure defined there is a random probability measure while $F_{W}$ is not random.

It remains to show convergence in probability, which is the type of convergence in Theorem 2.1 actually is sufficient to get Theorem 1.1 from this theorem. We do this by showing that every subsequence $\left\{\zeta_{k}\right\}$ of the positive integers contains yet another subsequence $\left\{\zeta_{k_{j}}\right\}$ so that $S\left(\zeta_{k_{j}}\right)$ converges in distribution to the same limit $W$ as $j \rightarrow \infty$. Then it follows easily (by the well known fact that a sequence of reals converges to a constant $a$ if and only if every subsequence contains a subsequence that converges to $a$, see e.g. $[9$, Section 5.7]), that also $S(n) \stackrel{d}{\rightarrow} W$.

To show this we use the fact that if a random variable $X_{n} \stackrel{p}{\rightarrow} X$ as $n \rightarrow \infty$, then there is an increasing subsequence of the integers $\left\{\zeta_{k}, k \geq 1\right\}$, such that $X_{\zeta_{k}} \stackrel{a . s}{\rightarrow} X$ as $n \rightarrow \infty$, see e.g. [9, Section 5.3]. Using this fact together with the famous diagonal method, see e.g. [9, Section 5.8], we can 
for all subsequences $\left\{\zeta_{k}\right\}$ pick a subsequence $\left\{\eta_{j}\right\}$ so that (ii) in Theorem 2.1 above holds a.s. for all $x \in Q$. Since the set of rational numbers is dense in $\Re$, there are for all $x$ arbitrarily close rational numbers $r$ and $s, r<x<s$ so that $\sum_{h(v) \leq L} \mathbf{P}\left(\xi_{v}>s\right) \leq \sum_{h(v) \leq L} \mathbf{P}\left(\xi_{v}>x\right) \leq \sum_{h(v) \leq L} \mathbf{P}\left(\xi_{v}>r\right)$ and it follows easily that (ii) holds (for the sub-subsequence we picked) for all $x \in \Re$. Now we can pick yet another subsequence from this sub-subsequence so that (iii) holds a.s. and from this yet another subsequence so that (iv) holds a.s. That is for all subsequences $\left\{\zeta_{k}, k \geq 1\right\}$ we find a subsequence $\left\{\zeta_{k_{j}}\right\}$ so that $S\left(\zeta_{k_{j}}\right) \stackrel{d}{\rightarrow} W$ and thus (42) holds.

Alternatively one can use Skorohod's coupling theorem, e.g. [14, Theorem 3.30], that is one can assume that (ii),(iii) and (iv) hold a.s. since one can couple the vector of

$$
\left(\sum_{h(v) \leq L} \mathbf{P}\left(\xi_{v}>x\right), \sum_{h(v) \leq L} \mathbf{E}\left(\xi_{v} \mathbf{1}\left[\xi_{v} \leq c\right]\right), \sum_{h(v) \leq L} \operatorname{Var}\left(\xi_{v} \mathbf{1}\left[\xi_{v} \leq c\right]\right)\right),
$$

with a parallel vector that converges a.s..

Thus, Theorem 1.1 for $X_{v}(T)$ follows from (41). Now it follows easily that the result holds for $X_{e}(T)$ too. The only difference is that the root vertex is ignored so that $\Lambda_{i} \in \operatorname{Exp}\left(\frac{1}{L}\right)$ instead of $\Lambda_{i} \in \operatorname{Exp}\left(\frac{1}{L+1}\right)$ as for $X_{v}(T)$. However, it follows easily that this minor difference does not affect the calculations in the proofs. Alternatively we could see $\widehat{T}$ (the rooted forest with two tree components) as the tree $T$ with the root vertex deleted. Then there is a natural 1-1 correspondence between edges of $T$ and vertices of $\widehat{T}$ and this correspondence also preserves the record (and cutting) operations. Hence, $X_{e}(T)=X_{v}(\widehat{T})$. Thus, the proof of Theorem 1.1 is completed.

Proof of Theorem 2.1. For any $x>0$,

$$
\begin{aligned}
\mathbf{P}\left(\xi_{v}>x \mid \mathscr{G}_{L}\right) & =\mathbf{P}\left(e^{-m \lambda_{v}}>\frac{n x}{m n_{v}} \mid \mathscr{G}_{L}\right)=\mathbf{P}\left(\lambda_{v}<\frac{1}{m} \ln \frac{m n_{v}}{n x} \mid \mathscr{G}_{L}\right) \\
& =1-\exp \left(-\frac{1}{m} \ln _{+} \frac{m n_{v}}{n x}\right) .
\end{aligned}
$$

Thus, for every $x>0$,

$$
\mathbf{P}\left(\xi_{v}>x \mid \mathscr{G}_{L}\right) \leq \frac{1}{m} \ln _{+} \frac{m n_{v}}{n x} \leq \frac{1}{m} \ln _{+} \frac{m}{x} \rightarrow 0,
$$

which proves (i).

Now we start by calculating the expected values of $\sum_{h(v) \leq L} \mathbf{P}\left(\xi_{v}>x \mid \mathscr{G}_{L}\right)$, $\sum_{h(v) \leq L} \mathbf{E}\left(\xi_{v} \mathbf{1}\left[\xi_{v} \leq c\right] \mid \mathscr{G}_{L}\right)$ and $\sum_{h(v) \leq L} \operatorname{Var}\left(\xi_{v} \mathbf{1}\left[\xi_{v} \leq c\right] \mid \mathscr{G}_{L}\right)$. For each vertex $v$, where $U_{r, v}$ are the uniform random variables defined in Subsection 
1.2.3, let $Z_{k, v}:=\sum_{r=1}^{k} \ln U_{r, v}$. Below we skip the vertex index and just write $Z_{k}$, since for vertices $v$ on the same level $k$ the $Z_{k, v}$ 's are identically distributed. We also let $Y_{k}:=-Z_{k}$. Noting that

$$
n_{v} \leq n \prod_{r=1}^{k} U_{r, v} \leq n_{v}+k
$$

motivates the following notation of $\widehat{n_{v}}:=n \prod_{r=1}^{k} U_{r, v}$. We note that the $\sigma$ field $\mathscr{G}_{j}$ defined in the beginning of Section 2 equivalently can be defined as the $\sigma$-field generated by $\left\{\widehat{n_{v}}: h(v) \leq j\right\}$.

Hence, by using (45), for large $n$

$$
\begin{aligned}
\sum_{h(v) \leq L} \mathbf{P}\left(\xi_{v}>x \mid \mathscr{G}_{L}\right) & =\sum_{k=0}^{L} \sum_{h(v)=k} \frac{1}{m} \ln _{+} \frac{m n_{v}}{n x}\left(1+\mathcal{O}\left(\frac{\ln m}{m}\right)\right) \\
& =\sum_{k=1}^{L} \sum_{h(v)=k} \frac{1}{m} \ln _{+} \frac{m \widehat{n_{v}}}{n x}\left(1+\mathcal{O}\left(\frac{\ln m}{m}\right)\right)+o(1) .
\end{aligned}
$$

Thus, the expected value of (46) is

$$
\begin{aligned}
& (1+o(1)) \sum_{k=1}^{L} 2^{k} \mathbf{E} \frac{1}{m} \ln _{+} \frac{m \prod_{i=1}^{k} U_{i}}{x}+o(1) . \\
& =(1+o(1)) \sum_{k=1}^{L} 2^{k} \mathbf{E}\left(\frac{\left(\ln m-\ln x-Y_{k}\right)}{m} I\left\{Y_{k} \leq \ln m-\ln x\right\}\right)+o(1) .
\end{aligned}
$$

Using characteristic functions (or from standard results) it follows that $Y_{k} \in \Gamma(k, 1)$, whence,

$$
\begin{aligned}
(47) & =(1+o(1)) \sum_{k=1}^{L} \int_{0}^{\ln m-\ln x} 2^{k} \frac{(\ln m-\ln x-y)}{m} \frac{e^{-y} y^{k-1}}{(k-1) !} d y+o(1) \\
& =(1+o(1)) \int_{0}^{\ln m-\ln x} \sum_{k=1}^{L} 2^{k} \frac{(\ln m-\ln x-y)}{m} \frac{e^{-y} y^{k-1}}{(k-1) !} d y+o(1)
\end{aligned}
$$

We use the ratio test on the sum

$$
\sum_{k=L+1}^{\infty} \frac{2^{k} y^{k-1}}{(k-1) !}
$$

and note that if $n$ is large then $k>2 \ln m>2 y$ for all $k>L$ and thus (49) is $\mathcal{O}(1)$. 
Hence,

$$
\int_{0}^{\ln m-\ln x} \sum_{k=L+1}^{\infty} 2^{k} \frac{(\ln m-\ln x-y)}{m} \frac{e^{-y} y^{k-1}}{(k-1) !} d y=o(1) .
$$

The Taylor expansion of $e^{x}$ gives

$$
\begin{aligned}
(48) & =\int_{0}^{\ln m-\ln x} \frac{2 e^{y}(\ln m-\ln x-y)}{m} d y+o(1) \\
& =\int_{0}^{\ln m-\ln x} \frac{2 e^{\ln m-\ln x-u}}{m} d u+o(1)=\frac{2}{x} \int_{0}^{\ln m-\ln x} e^{-u} d u+o(1) \\
& =\frac{2}{x}+o(1)=\nu(x, \infty)+o(1) .
\end{aligned}
$$

Note that $\nu(x, \infty)$ is continuous and decreasing with $\nu(x, \infty) \rightarrow 0$ as $x \rightarrow \infty$. The derivative is

$$
\frac{d \nu(x, \infty)}{d x}=\frac{-2}{x^{2}}
$$

For (iii) in Theorem 2.1 it is easy to calculate (compare with [11, p.251]) that

$$
\begin{aligned}
\sum_{h(v) \leq L} \mathbf{E}\left(\xi_{v} \mathbf{1}\left[\xi_{v} \leq c\right] \mid \mathscr{G}_{L}\right) & =\sum_{h(v) \leq L} \frac{m n_{v}}{n} \mathbf{E}\left(e^{-m \lambda_{v}} \mathbf{1}\left[e^{-m \lambda_{v}} \leq \frac{n c}{m n_{v}}\right] \mid \mathscr{G}_{L}\right) \\
& =\sum_{h(v) \leq L} \frac{m n_{v}}{n(m+1)} e^{-\frac{m+1}{m} \ln _{+}\left(\frac{m n_{v}}{n c}\right)} .
\end{aligned}
$$

By using (45) one gets

$$
(52)=\sum_{h(v) \leq L} \frac{m \widehat{n_{v}}}{n(m+1)} e^{-\frac{m+1}{m} \ln +\left(\frac{m \widehat{n_{v}}}{n c}\right)}+o(1) .
$$

Thus, the expected value of (52) is

$$
\begin{gathered}
\mathbf{E} \sum_{h(v) \leq L} \frac{m \prod_{r=1}^{k} U_{r, v}}{m+1} e^{-\frac{m+1}{m} \ln \left(\frac{m \prod_{r=1}^{k} U_{r, v}}{c}\right) I\left\{\prod_{r=1}^{k} U_{r, v} \geq \ln c-\ln m\right\}}+o(1) \\
=\mathbf{E} \sum_{h(v) \leq L} \frac{m}{m+1} e^{-Y_{k}} e^{-\frac{m+1}{m}\left(\ln m-\ln c-Y_{k}\right) I\left\{Y_{k} \leq \ln m-\ln c\right\}}+o(1) \\
=\mathbf{E} \sum_{h(v) \leq L} \frac{m}{m+1} e^{-Y_{k}} e^{-\frac{m+1}{m}\left(\ln m-\ln c-Y_{k}\right)} I\left\{Y_{k} \leq \ln m-\ln c\right\} \\
\quad+\mathbf{E} \sum_{h(v) \leq L} \frac{m}{m+1} e^{-Y_{k}} I\left\{Y_{k}>\ln m-\ln c\right\}+o(1) .
\end{gathered}
$$


$Y_{k} \in \Gamma(k, 1)$ implies,

$$
(54)=E_{1}+E_{2}+o(1)
$$

where

$$
\begin{aligned}
& E_{1}:=\sum_{k=1}^{L} 2^{k} \int_{0}^{\ln m-\ln c} \frac{m}{m+1} e^{-y} e^{-\frac{m+1}{m}(\ln m-\ln c-y)} \frac{e^{-y} y^{k-1}}{(k-1) !}, \\
& E_{2}:=\mathbf{E} \sum_{k=1}^{L} 2^{k} \frac{m}{m+1} \prod_{r=1}^{k} U_{r, v}\left(1-I\left\{Y_{k} \leq \ln m-\ln c\right\}\right) .
\end{aligned}
$$

We again use the ratio test on the sum in (49) and by a similar argument that is used to show that (50) gives (51) one gets from the Taylor expansion of $e^{x}$,

$$
\begin{aligned}
E_{1} & =\int_{0}^{\ln m-\ln c} \frac{m}{m+1} \sum_{k=1}^{L} 2^{k} e^{-2 y} e^{-\frac{m+1}{m}(\ln m-\ln c-y)} \frac{y^{k-1}}{(k-1) !} d y+o(1) \\
& =\int_{0}^{\ln m-\ln c} \frac{2 m}{m+1} e^{-\frac{m+1}{m}(\ln m-\ln c-y)} d y+o(1) \\
& =\left|\frac{2 m^{2}}{(m+1)^{2}} e^{-\frac{m+1}{m}(\ln m-\ln c-y)}\right|_{0}^{\ln m-\ln c}+o(1)=2+o(1)
\end{aligned}
$$

and

$$
\begin{aligned}
E_{2} & =\frac{m}{m+1} L-\int_{0}^{\ln m-\ln c} \frac{m}{m+1} \sum_{k=1}^{L} 2^{k} e^{-2 y} \frac{y^{k-1}}{(k-1) !} d y+o(1) \\
& =L-\left|\frac{2 m y}{m+1}\right|_{0}^{\ln m-\ln c}+o(1) \\
& =L-2 \ln m+2 \ln c+o(1) .
\end{aligned}
$$

Thus,

$(54)=L+2-2 \ln m+2 \ln c+o(1)=L+2-2 \ln \ln n-2 \ln 2+2 \ln c+o(1)$.

For $\sum_{h(v)=L} \frac{n_{v}}{2 \ln n_{v}}$ (since we can ignore those $n_{v}<\frac{n}{2^{k L}}$ for $k$ large enough) 
we have the expansion

$$
\begin{aligned}
\sum_{h(v)=L} \frac{n_{v}}{2 \ln n_{v}} & =\sum_{h(v)=L} \frac{n_{v}}{2 \ln n\left(1+\frac{\ln \left(\frac{n_{v}}{n}\right)}{\ln n}\right)} \\
& =\sum_{h(v)=L} \frac{n_{v}}{2 \ln n}-\sum_{h(v)=L} \frac{n_{v} \ln \left(\frac{n_{v}}{n}\right)}{2 \ln ^{2} n}+o\left(\frac{n}{\ln ^{2} n}\right) \\
& =\frac{n}{2 \ln n}-\sum_{h(v)=L} \frac{n_{v} \ln \left(\frac{n_{v}}{n}\right)}{2 \ln ^{2} n}+o\left(\frac{n}{\ln ^{2} n}\right) .
\end{aligned}
$$

Using (45) again gives

$$
\begin{aligned}
\sum_{h(v)=L} \frac{n_{v}}{2 \ln n_{v}} & =\frac{n}{2 \ln n}-\sum_{h(v)=L} \frac{\widehat{n_{v}} \ln \left(\frac{\widehat{n_{v}}}{n}\right)}{2 \ln ^{2} n}+o\left(\frac{n}{\ln ^{2} n}\right) \\
& =\frac{n}{2 \ln n}-\sum_{h(v)=L} \frac{n \prod_{r=1}^{L} U_{r, v} \sum_{r=1}^{L} \ln U_{r, v}}{2 \ln ^{2} n}+o\left(\frac{n}{\ln ^{2} n}\right) .
\end{aligned}
$$

Taking expectation of (60) we get

$$
\frac{n}{2 \ln n}+\frac{n L}{4 \ln ^{2} n}+o\left(\frac{n}{\ln ^{2} n}\right)
$$

For $(i v)$ in Theorem 2.1 by using the calculations in [11, p.251-252] we get

$$
\begin{aligned}
\sum_{h(v) \leq L} \operatorname{Var}\left(\xi_{v} \mathbf{1}\left[\xi_{v} \leq c\right] \mid \mathscr{G}_{L}\right) & =\sum_{h(v) \leq L} \frac{m^{2} n_{v}{ }^{2}}{n^{2}} \operatorname{Var}\left(e^{-m \lambda_{v}} \mathbf{1}\left[e^{-m \lambda_{v}} \leq \frac{n c}{m n_{v}}\right] \mid \mathscr{G}_{L}\right) \\
& =\sum_{h(v) \leq L} \frac{m^{2} n_{v}{ }^{2}}{2 m n^{2}} e^{-\frac{2 m+1}{m} \ln _{+}\left(\frac{m n_{v}}{n c}\right)}+o(1)
\end{aligned}
$$

Using (45) one obtains,

$$
(62)=\sum_{h(v) \leq L} \frac{m^{2} \widehat{n_{v}}}{2 m n^{2}} e^{-\frac{2 m+1}{m} \ln _{+}\left(\frac{m \widehat{n v}}{n c}\right)}+o(1) .
$$

Hence, by using $Y_{k} \in \Gamma(k, 1)$ and taking expectation of $(62)$ one gets

$$
\sum_{k=1}^{L} \frac{2^{k} m}{2} \mathbf{E}\left(\prod_{r=1}^{k} U_{r, v}^{2} e^{-\frac{2 m+1}{m}\left(\ln m-\ln c-Y_{k}\right) I\left\{Y_{k} \leq \ln m-\ln c\right\}}\right)+o(1)=V_{1}+V_{2}+o(1),
$$


where

$$
\begin{aligned}
V_{1} & :=\sum_{k=1}^{L} \frac{2^{k} m}{2} \int_{0}^{\ln m-\ln c} e^{-2 y} e^{-\frac{2 m+1}{m}(\ln m-\ln c-y)} \frac{e^{-y} y^{k-1}}{(k-1) !} d y, \\
V_{2} & :=\mathbf{E}\left(\sum_{k=1}^{L} \frac{2^{k} m}{2} \prod_{r=1}^{k} U_{r, v}^{2}\left(1-I\left\{Y_{k} \leq \ln m-\ln c\right\}\right)\right) .
\end{aligned}
$$

Again we use the ratio test on the sum in (49) and the Taylor expansion of $e^{x}$ to get

$$
\begin{aligned}
V_{1} & =\int_{0}^{\ln m-\ln c} m e^{-\frac{2 m+1}{m}(\ln m-\ln c)} e^{\frac{m+1}{m} y} d y+o(1)=\frac{m^{2}}{m+1} e^{(\ln m-\ln c)}+o(1) \\
& =c+o(1)
\end{aligned}
$$

and

$$
\begin{aligned}
& V_{2}=\sum_{k=1}^{L} \frac{2^{k-1} m}{3^{k}}-\int_{0}^{\ln m-\ln c} \sum_{k=1}^{L} e^{-2 y} \frac{e^{-y} 2^{k-1} m y^{k-1}}{(k-1) !} d y+o(1) \\
& =m-\int_{0}^{\ln m-\ln c} m e^{-y} d y+o(1)=c+o(1) .
\end{aligned}
$$

We have now proved that the expected values of the left hand sides $(i i)$, (iii) and $(i v)$ in Theorem 2.1 converge to the right hand sides.

We complete the proof of Theorem 2.1 by showing,

$$
\begin{aligned}
& \operatorname{Var} \sum_{h(v) \leq L} \mathbf{P}\left(\xi_{v}>x \mid \mathscr{G}_{L}\right) \rightarrow 0 \text { for every } x>0, \\
& \operatorname{Var}\left(\sum_{h(v) \leq L} \mathbf{E}\left(\xi_{v} \mathbf{1}\left[\xi_{v} \leq c\right] \mid \mathscr{G}_{L}\right)-\frac{4 \ln ^{2} n}{n} \sum_{h(v)=L} \frac{n_{v}}{2 \ln n_{v}}\right) \rightarrow 0, \\
& \operatorname{Var} \sum_{h(v) \leq L} \operatorname{Var}\left(\xi_{v} \mathbf{1}\left[\xi_{v} \leq c\right] \mid \mathscr{G}_{L}\right) \rightarrow 0 .
\end{aligned}
$$

Then by Chebyshev's inequality $(i i),(i i i)$ and $(i v)$ in Theorem 2.1 follow.

In preparation to show (68), (69) and (70), defining $l$ as $l:=\lfloor 100 \ln \ln \ln n\rfloor$ we note that by using (44) one easily obtains (see [11, equations (19), (21), 


$$
\begin{aligned}
\sum_{h(v) \leq L} \mathbf{P}\left(\xi_{v}>x \mid \mathscr{G}_{L}\right) & =\sum_{l \leq h(v) \leq L} \mathbf{P}\left(\xi_{v}>x \mid \mathscr{G}_{L}\right)+o(1), \\
\sum_{h(v) \leq L} \mathbf{E}\left(\xi_{v} \mathbf{1}\left[\xi_{v} \leq c\right] \mid \mathscr{G}_{L}\right) & =\sum_{l \leq h(v) \leq L} \mathbf{E}\left(\xi_{v} \mathbf{1}\left[\xi_{v} \leq c\right] \mid \mathscr{G}_{L}\right)+o(1), \\
\sum_{h(v) \leq L} \operatorname{Var}\left(\xi_{v} \mathbf{1}\left[\xi_{v} \leq c\right] \mid \mathscr{G}_{L}\right) & =\sum_{l \leq h(v) \leq L} \mathbf{V a r}\left(\xi_{v} \mathbf{1}\left[\xi_{v} \leq c\right] \mid \mathscr{G}_{L}\right)+o(1) .
\end{aligned}
$$

(In fact one can choose a larger value for $l$ in (71), (72) and (73), that is $\rho \ln \ln n$ for some constant $\rho$.)

To show (68), (69) and (70) we use a variance formula that is easy to establish, see e.g. [9, exercise 10.17-2],

$$
\operatorname{Var}(Y)=\mathbf{E}(\operatorname{Var}(Y \mid \mathscr{G}))+\operatorname{Var}(\mathbf{E}(Y \mid \mathscr{G})),
$$

where $Y$ is a random variable and $\mathscr{G}$ is a sub- $\sigma$-field. From the definition of $\mathscr{G}_{j}$ we get that in particular $\mathscr{G}_{l}$ is the $\sigma$-field generated by $\left\{\widehat{n_{v}}: h(v) \leq l\right\}$. (All the conditional expected values below are as always defined a.s. but we do not write it out.)

Consequently,

$$
\begin{aligned}
\operatorname{Var}\left(\sum_{l \leq h(v) \leq L} \mathbf{P}\left(\xi_{v}>x \mid \mathscr{G}_{L}\right) \mid \mathscr{G}_{l}\right) \\
=\mathbf{E}\left(\operatorname{Var}\left(\sum_{l \leq h(v) \leq L} \mathbf{P}\left(\xi_{v}>x \mid \mathscr{G}_{L}\right) \mid \mathscr{G}_{l}\right)\right) \\
\quad+\operatorname{Var}\left(\mathbf{E}\left(\sum_{l \leq h(v) \leq L} \mathbf{P}\left(\xi_{v}>x \mid \mathscr{G}_{L}\right) \mid \mathscr{G}_{l}\right)\right) .
\end{aligned}
$$

For a given vertex $v_{i} \in T$ with $h\left(v_{i}\right)=l$, there are at most $2^{j-l}$ choices of $v$ at height $j$ with ancestor $v_{i}$. From Subsection 1.2.3 and the notations introduced in the beginning of this proof $\widehat{n_{v_{i}}}=n \prod_{r=1}^{l} U_{r, v_{i}}, \widehat{n_{v}}=n \prod_{r=1}^{j} U_{r, v}$, $Z_{l}:=\sum_{r=1}^{l} \ln U_{r, v}$, and $Y_{l, v_{i}}:=-\sum_{r=1}^{l} \ln U_{r, v_{i}}$. For $v$ with $h(v)=j$, we also define $Y_{j-l, v}:=-\sum_{r=l+1}^{j} \ln U_{r, v}$.

Since $Y_{k} \in \Gamma(k, 1)$ (by using $(71)$ ) we get by similar calculations as in 
$(46)-(51)$,

$$
\begin{aligned}
& \mathbf{E}\left(\sum_{l \leq h(v) \leq L} \mathbf{P}\left(\xi_{v}>x \mid \mathscr{G}_{L}\right) \mid \mathscr{G}_{l}\right) \\
& =(1+o(1)) \sum_{i=1}^{2^{l}} \int_{0}^{\ln m-\ln x-Y_{l, v_{i}}} \sum_{j=l+1}^{L} 2^{j-l} \frac{\left(\ln m-\ln x-Y_{l, v_{i}}-y_{j-l, v}\right)}{m} \frac{e^{-y_{j-l, v}} y_{j-l, v}^{j-l-1}}{(j-l-1) !} d y_{j-l, v} \\
& \quad+o(1) \\
& =\sum_{i=1}^{2^{l}} \int_{0}^{\ln m-\ln x-Y_{l, v_{i}}} \frac{2 e^{y_{j-l, v}}\left(\ln m-\ln x-Y_{l, v_{i}}-y_{j-l, v}\right)}{m} d y_{j-l, v}+o(1) \\
& =\sum_{i=1}^{2^{l}} \int_{0}^{\ln m-\ln x-Y_{l, v_{i}}} \frac{2 e^{y_{j-l, v}}}{m} d y_{j-l, v}+o(1)=\sum_{i=1}^{2^{l}} \prod_{r=1}^{l} U_{r, v_{i}} \frac{2}{x}+o(1)=\frac{2}{x}+o(1) .
\end{aligned}
$$

Thus,

$$
\operatorname{Var}\left(\mathbf{E}\left(\sum_{l \leq h(v) \leq L} \mathbf{P}\left(\xi_{v}>x \mid \mathscr{G}_{L}\right) \mid \mathscr{G}_{l}\right)\right) \rightarrow 0
$$

We proceed by analyzing (75) in the variance formula above.

$$
\begin{aligned}
& \operatorname{Var}\left(\sum_{l \leq h(v) \leq L} \mathbf{P}\left(\xi_{v}>x \mid \mathscr{G}_{L}\right) \mid \mathscr{G}_{l}\right) \\
& =\sum_{\substack{l \leq h(v) \leq L, l \leq h(w) \leq L}} \operatorname{Cov}\left(\mathbf{P}\left(\xi_{v}>x \mid \mathscr{G}_{L}\right), \mathbf{P}\left(\xi_{w}>x \mid \mathscr{G}_{L}\right) \mid \mathscr{G}_{l}\right) .
\end{aligned}
$$

To calculate these conditional covariances we can suppose that the closest ancestor $u$ for $v$ at $h(v) \leq L$ and $w$ at $h(w) \leq L$ is at height $h \geq l$ since the other terms are just 0 because of independence.

For $h \geq l$ we use

$$
\operatorname{Cov}\left(\mathbf{P}\left(\xi_{v}>x \mid \mathscr{G}_{L}\right), \mathbf{P}\left(\xi_{w}>x \mid \mathscr{G}_{L}\right) \mid \mathscr{G}_{l}\right) \leq \mathbf{E}\left(\mathbf{P}\left(\xi_{v}>x \mid \mathscr{G}_{L}\right)\left(\mathbf{P}\left(\xi_{w}>x \mid \mathscr{G}_{L}\right) \mid \mathscr{G}_{l}\right),\right.
$$

which implies

$$
\mathbf{E}\left(\operatorname{Cov}\left(\mathbf{P}\left(\xi_{v}>x \mid \mathscr{G}_{L}\right), \mathbf{P}\left(\xi_{w}>x \mid \mathscr{G}_{L}\right) \mid \mathscr{G}_{l}\right)\right) \leq \mathbf{E}\left(\mathbf{P}\left(\xi_{v}>x \mid \mathscr{G}_{L}\right)\left(\mathbf{P}\left(\xi_{w}>x \mid \mathscr{G}_{L}\right)\right) .\right.
$$


Denote by $\left(v_{u}, w_{u}\right)$ a general pair of vertices with closest ancestor $u$. Then by using (81) we get,

$$
(75) \leq \sum_{h=l}^{L} \sum_{u: h(u)=h} \sum_{\left(v_{u}, w_{u}\right)} \mathbf{E}\left(\mathbf{P}\left(\xi_{v_{u}}>x \mid \mathscr{G}_{L}\right) \cdot\left(\mathbf{P}\left(\xi_{w_{u}}>x \mid \mathscr{G}_{L}\right)\right) .\right.
$$

For the pair $v_{u}, w_{u}$ with $h(u)=h$ we get that if we condition on $\mathscr{G}_{h+1}$ (the $\sigma$-field generated by $\left.\left\{\widehat{n_{v}}: h(v) \leq h+1\right\}\right)$ then, $\mathbf{E}\left(\mathbf{P}\left(\xi_{v_{u}}>x \mid \mathscr{G}_{L}\right) \mid \mathscr{G}_{h+1}\right)$ and $\mathbf{E}\left(\mathbf{P}\left(\xi_{w_{u}}>x \mid \mathscr{G}_{L}\right) \mid \mathscr{G}_{h+1}\right)$ are independent. Thus,

$$
\begin{aligned}
& \mathbf{E}\left(\mathbf{P}\left(\xi_{v_{u}}>x \mid \mathscr{G}_{L}\right) \mathbf{P}\left(\xi_{w_{u}}>x \mid \mathscr{G}_{L}\right) \mid \mathscr{G}_{h+1}\right) \\
& =\mathbf{E}\left(\mathbf{P}\left(\xi_{v_{u}}>x \mid \mathscr{G}_{L}\right) \mid \mathscr{G}_{h+1}\right) \cdot \mathbf{E}\left(\mathbf{P}\left(\xi_{w_{u}}>x \mid \mathscr{G}_{L}\right) \mid \mathscr{G}_{h+1}\right) .
\end{aligned}
$$

Denote by $v, w: v \wedge w=u$ that the vertices $v, w$ have closest ancestor $u$. Using (43) and (44), by similar calculations as in (47) for $v, w$ and $u$ with $h(v)=j, h(w)=k$ and $h(u)=h$ respectively (where $u$ is the closest ancestor vertex to $v$ and $w)$ we get

$$
\begin{aligned}
& \sum_{h=l}^{L} \sum_{u: h(u)=h} \sum_{v, w \in T_{u}: v \wedge w=u} \mathbf{E}\left(\mathbf{P}\left(\xi_{v}>x \mid \mathscr{G}_{L}\right) \cdot\left(\mathbf{P}\left(\xi_{w}>x \mid \mathscr{G}_{L}\right) \mid \mathscr{G}_{h+1}\right)\right.
\end{aligned}
$$

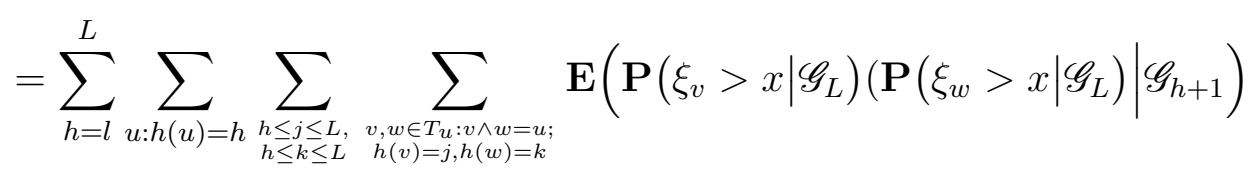

$$
\begin{aligned}
& \leq \sum_{h=l}^{L} \sum_{\substack{u: h(u)=h \\
\sum_{h}}} \sum_{\substack{h \leq j \leq L, h \leq k \leq L}} \\
& \mathbf{E}\left(\frac{1}{m} \ln _{+}\left(\frac{m \prod_{r=1}^{j} U_{r, v}}{x}\right) \frac{1}{m} \ln _{+}\left(\frac{m \prod_{r=1}^{k} U_{r, w}}{x}\right) \mid \mathscr{G}_{h+1}\right) \\
& \leq \sum_{h=l}^{L} \sum_{u: h(u)=h} \sum_{j=h}^{L} \sum_{\substack{v \in T_{u}: \\
h(v)=j}} \mathbf{E}\left(\frac{1}{m} \ln _{+} \frac{m \prod_{r=1}^{j} U_{r, v}}{x} \mid \mathscr{G}_{h+1}\right) \\
& \cdot \sum_{h=l}^{L} \sum_{u: h(u)=h} \sum_{k=h}^{L} \sum_{\substack{w \in T_{u}: \\
h(w)=k}} \mathbf{E}\left(\frac{1}{m} \ln _{+} \frac{m \prod_{r=1}^{k} U_{r, w}}{x} \mid \mathscr{G}_{h+1}\right) .
\end{aligned}
$$

Note that the expected value of (84) is (82). 
From the notations in Subsection 1.2.3 we get for a triple $(v, w, u)$ with $h(v)=j, h(w)=k, h(u)=h$ that

$$
\begin{array}{r}
\widehat{n_{v}}=n \prod_{r=1}^{j} U_{r, v}=n \prod_{r=1}^{h} U_{r, u} \widehat{\mathcal{U}}_{u} \prod_{r=h+2}^{j} U_{r, v}, \\
\widehat{n_{w}}=n \prod_{r=1}^{k} U_{r, w}=n \prod_{r=1}^{h} U_{r, u}\left(1-\widehat{\mathcal{U}}_{u}\right) \prod_{r=h+2}^{k} U_{r, w},
\end{array}
$$

where $\widehat{\mathcal{U}}_{u}$ is either $\mathcal{U}_{u}$ or $1-\mathcal{U}_{u}$ depending on if $v$ is to the right or left of $w$. For given $h(u)=h \geq l, h(v)=j$ and $h(w)=k$ there are at most $2^{h}$ choices of $u$ and then at most $2^{j-h}$ choices of $v$ and $2^{k-h}$ choices of $w$. (We can assume that $j>h+1$ and $k>h+1$, since it is easy to see that the other terms are few and the sum of them is small.) Using the notations in Subsection 1.2.3, we define for a vertex $u$ with $h(u)=h$ (the closest ancestor vertex of a pair $(v, w)) Y_{h+1, u}:=-\sum_{r=1}^{h} \ln U_{r, u}-\ln \widehat{\mathcal{U}}_{u}, \widehat{Y}_{h+1, u}:=-\sum_{r=1}^{h} \ln U_{r, u}-\ln \left(1-\widehat{\mathcal{U}}_{u}\right)$ and for the vertex $v$ with $h(v)=j$ we let $Y_{j-h-1, v}:=-\sum_{r=h+2}^{j} \ln U_{r, v}$ (and use the corresponding notation for $w$ with $h(w)=k$ ). (For simplicity we skip the vertex index in the calculations below except for the vertices $v_{i}$ at height l.)

Thus, by similar calculations as in (48) and (51),

$$
\begin{aligned}
(84)= & \frac{1}{m^{2}} O\left(\sum _ { h = l } ^ { L } \sum _ { h ( u ) = h } \left(\sum_{j=h+2}^{L} \int_{0}^{\ln m-\ln x-Y_{h+1}}\right.\right. \\
& \left(2^{j-h-1}\left(\ln m-\ln x-Y_{h+1}-y_{j-h-1}\right) \frac{\left.\left.e^{-y_{j-h-1}} y_{j-h-1}^{j-h-2}\right) d y_{j-h-1}\right) .}{(j-h-2) !}\right. \\
& \left.\left(\sum_{k=h+2}^{L} \int_{0}^{\ln m-\ln x-\widehat{Y}_{h+1}} 2^{k-h-1}\left(\ln m-\ln x-\widehat{Y}_{h+1}-y_{k-h-1}\right) \frac{e^{-y_{k-h-1}} y_{k-h-1}^{k-h-2}}{(k-h-2) !} d y_{k-h-1}\right)\right) \\
= & \mathcal{O}\left(\sum_{h=l}^{L} \sum_{h(u)=h} \frac{1}{m^{2}}\left(\int_{0}^{\ln m-\ln x-Y_{h}} e^{y} d y\right)^{2}\right) \\
= & \mathcal{O}\left(\sum_{h=l}^{L} \sum_{h(u)=h} \frac{1}{x^{2}} \prod_{j=1}^{h} U_{j}^{2}\right) .
\end{aligned}
$$

The expected value of (86) is

$$
\mathcal{O}\left(\sum_{h=l}^{L} \frac{2^{h}}{3^{h}} \frac{1}{x^{2}}\right)=o(1),
$$


and thus $(82)=o(1)$.

This implies by using that $(75) \leq(82)$

$$
\mathbf{E}\left(\operatorname{Var}\left(\sum_{l \leq h(v) \leq L} \mathbf{P}\left(\xi_{v}>x \mid \mathscr{G}_{L}\right) \mid \mathscr{G}_{l}\right)\right) \rightarrow 0
$$

which shows (68).

We show that (69) is true by similar calculations as for (68). First, as before let $v_{i}$ with $h\left(v_{i}\right)=l$ be a given vertex so that there are at most $2^{j-l}$ choices of $v$ at height $j$ with ancestor $v_{i}$. Recall the notations introduced to prove (78). The notations above also give $Z_{j, v}=Z_{l, v_{i}}+Z_{j-l, v}$ where $Z_{l, v_{i}}:=-Y_{l, v_{i}}$ and $Z_{j-l, v}:=-Y_{j-l, v}$.

Using (72) by similar calculations as in (77) (glancing at the calculations in (52), (54) and (55)) we obtain

$$
\begin{gathered}
\mathbf{E}\left(\sum_{l \leq h(v) \leq L} \mathbf{E}\left(\xi_{v} \mathbf{1}\left[\xi_{v} \leq c\right] \mid \mathscr{G}_{L}\right) \mid \mathscr{G}_{l}\right) \\
=\mathbf{E}\left(\sum_{l \leq h(v) \leq L} \frac{m}{m+1} e^{-Y_{l, v_{i}}-Y_{j-l, v}} e^{-\frac{m+1}{m}\left(\ln m-\ln c-Y_{l, v_{i}}-Y_{j-l, v}\right)}\right. \\
\left.I\left\{Y_{l, v_{i}}+Y_{j-l, v} \leq \ln m-\ln c\right\} \mid \mathscr{G}_{l}\right) \\
+\mathbf{E}\left(\sum_{l \leq h(v) \leq L} \frac{m}{m+1} e^{-Y_{l, v_{i}}-Y_{j-l, v}} I\left\{Y_{l, v_{i}}+Y_{j-l, v}>\ln m-\ln c\right\} \mid \mathscr{G}_{l}\right)+o(1) \\
=F_{1}+F_{2}+o(1),
\end{gathered}
$$

where

$$
\begin{aligned}
F_{1} & :=\sum_{i=1}^{2^{l}} \sum_{j=l+1}^{L} 2^{j-l}\left(\int_{0}^{\ln m-\ln c-Y_{l, v_{i}}} \frac{m}{m+1} e^{-Y_{l, v_{i}}-y_{j-l, v}}\right. \\
& \left.\cdot e^{-\frac{m+1}{m}\left(\ln m-\ln c-Y_{l, v_{i}}-y_{j-l, v}\right)} \frac{e^{-y_{j-l, v}} y_{j-l, v}^{j-l-1}}{(j-l-1) !} d y_{j-l, v}\right)
\end{aligned}
$$

and

$$
F_{2}:=\sum_{i=1}^{2^{l}} \mathbf{E}\left(\sum_{j=l+1}^{L} 2^{j-l} \frac{m}{m+1} \prod_{r=1}^{l} U_{r, v_{i}} \prod_{r=l+1}^{j} U_{r, v}\left(1-I\left\{Y_{l, v_{i}}+Y_{j-l, v} \leq \ln m-\ln c\right\}\right) \mid \mathscr{G}_{l}\right) .
$$


Now using similar calculations as in (56) we obtain

$$
\begin{aligned}
F_{1}= & \sum_{i=1}^{2^{l}} \int_{0}^{\ln m-\ln c-Y_{l, v_{i}}}\left(\frac{m}{m+1} \sum_{j=l+1}^{L} 2^{j-l} e^{-Y_{l, v_{i}}-2 y_{j-l, v}}\right. \\
& \left.\cdot e^{-\frac{m+1}{m}\left(\ln m-\ln c-Y_{l, v_{i}}-y_{j-l, v}\right)} \frac{y_{j-l, v}^{j-l-1}}{(j-l-1) !}\right) d y_{j-l, v} \\
= & \sum_{i=1}^{2^{l}} \int_{0}^{\ln m-\ln c-Y_{l, v_{i}}} e^{-Y_{l, v_{i}}} \frac{2 m}{m+1} e^{-\frac{m+1}{m}\left(\ln m-\ln c-Y_{l, v_{i}}-y_{j-l, v}\right)} d y_{j-l, v}+o(1) \\
= & \sum_{i=1}^{2^{l}} 2 \prod_{r=1}^{l} U_{r, v_{i}}+o(1)=2+o(1) .
\end{aligned}
$$

By using similar calculations as in (55) and (57) we obtain

$$
\begin{aligned}
F_{2}= & \sum_{i=1}^{2^{l}}\left(\prod_{r=1}^{l} U_{r, v_{i}}(L-l)-\int_{0}^{\ln m-\ln c-Y_{l, v_{i}}}\left(\frac{m}{m+1} \sum_{j=l+1}^{L} 2^{j-l}\right.\right. \\
& \left.\left.\cdot e^{-Y_{l, v_{i}}-2 y_{j-l, v}} \frac{y_{j-l, v}^{j-l-1}}{(j-l-1) !}\right) d y_{j-l, v}\right)+o(1) \\
= & \sum_{i=1}^{2^{l}} \prod_{r=1}^{l} U_{r, v_{i}}\left((L-l)-\left|\frac{2 m y_{j-l, v}}{m+1}\right|_{0}^{\ln m-\ln c-Y_{l, v_{i}}}\right)+o(1) \\
= & \sum_{i=1}^{2^{l}} \prod_{r=1}^{l} U_{r, v_{i}}\left(L-l-2 \ln m+2 \ln c-2 \sum_{r=1}^{l} \ln U_{r, v_{i}}\right)+o(1) \\
= & L-l-2 \ln m+2 \ln c-\sum_{i=1}^{2^{l}} 2 \prod_{r=1}^{l} U_{r, v_{i}} \sum_{r=1}^{l} \ln U_{r, v_{i}}+o(1) .
\end{aligned}
$$

Thus,

$$
\begin{aligned}
& \mathbf{E}\left(\sum_{l \leq h(v) \leq L} \mathbf{E}\left(\xi_{v} \mathbf{1}\left[\xi_{v} \leq c\right] \mid \mathscr{G}_{L}\right) \mid \mathscr{G}_{l}\right) \\
& =2+L-l-2 \ln m+2 \ln c-\sum_{i=1}^{2^{l}} 2 \prod_{r=1}^{l} U_{r, v_{i}} \sum_{r=1}^{l} \ln U_{r, v_{i}}+o(1) .
\end{aligned}
$$

We now use the expansion of $\sum_{h(v)=L} \frac{n_{v}}{2 \ln n_{v}}$ in (59) and (60) keeping the notations used in earlier calculations. Let $v_{i}$ be a vertex at height $l$ and let 
$v$ now be a vertex at the fixed level $L$. Thus, using (45)

$$
\begin{gathered}
\mathbf{E}\left(\sum_{h(v)=L} \frac{n_{v}}{2 \ln n_{v}} \mid \mathscr{G}_{l}\right)=\mathbf{E}\left(\sum_{h(v)=L} \frac{\widehat{n_{v}}}{2 \ln \widehat{n_{v}}} \mid \mathscr{G}_{l}\right)+o\left(\frac{n}{\ln ^{2} n}\right) \\
=\mathbf{E}\left(\frac{n}{2 \ln n}-\sum_{i=1}^{2^{l}} \prod_{r=1}^{l} U_{r, v_{i}}\left(2 ^ { L - l } \left(\frac{n \prod_{r=l+1}^{L} U_{r, v} \sum_{r=l+1}^{L} \ln U_{r, v}}{2 \ln ^{2} n}\right.\right.\right. \\
\left.\left.\left.+\frac{n \prod_{r=l+1}^{L} U_{r, v} \sum_{r=1}^{l} \ln U_{r, v_{i}}}{2 \ln ^{2} n}\right)\right) \mid \mathscr{G}_{l}\right)+o\left(\frac{n}{\ln ^{2} n}\right) .
\end{gathered}
$$

Thus, the conditional expected value in (95) given $\mathscr{G}_{l}$ is (similar to (61))

$$
\begin{aligned}
& \mathbf{E}\left(\sum_{h(v)=L} \frac{n_{v}}{2 \ln n_{v}} \mid \mathscr{G}_{l}\right)= \\
& \frac{n}{2 \ln n}+\sum_{i=1}^{2^{l}} \prod_{r=1}^{l} U_{r, v_{i}} \frac{n(L-l)}{4 \ln ^{2} n}-\sum_{i=1}^{2^{l}} \frac{n \prod_{r=1}^{l} U_{r, v_{i}} \sum_{r=1}^{l} \ln U_{r, v_{i}}}{2 \ln ^{2} n}+o\left(\frac{n}{\ln ^{2} n}\right) \\
& =\frac{n}{2 \ln n}+\frac{n(L-l)}{4 \ln ^{2} n}-\sum_{i=1}^{2^{l}} \frac{n \prod_{r=1}^{l} U_{r, v_{i}} \sum_{r=1}^{l} \ln U_{r, v_{i}}}{2 \ln ^{2} n}+o\left(\frac{n}{\ln ^{2} n}\right)
\end{aligned}
$$

From (94) and (96) we obtain

$$
\operatorname{Var}\left(\mathbf{E}\left(\sum_{l \leq h(v) \leq L} \mathbf{E}\left(\xi_{v} \mathbf{1}\left[\xi_{v} \leq c\right] \mid \mathscr{G}_{L}\right)-\frac{4 \ln ^{2} n}{n} \sum_{h(v)=L} \frac{n_{v}}{2 \ln n_{v}} \mid \mathscr{G}_{l}\right)\right) \rightarrow 0
$$

We proceed with the first term on the right hand side of the variance formula in (74). First we consider

$$
\begin{aligned}
& \operatorname{Var}\left(\sum_{l \leq h(v) \leq L} \mathbf{E}\left(\xi_{v} \mathbf{1}\left[\xi_{v} \leq c\right] \mid \mathscr{G}_{L}\right) \mid \mathscr{G}_{l}\right) \\
& =\sum_{\substack{l \leq h(v) \leq L, l \leq h(w) \leq L}} \operatorname{Cov}\left(\mathbf{E}\left(\xi_{v} \mathbf{1}\left[\xi_{v} \leq c\right] \mid \mathscr{G}_{L}\right), \mathbf{E}\left(\xi_{w} \mathbf{1}\left[\xi_{w} \leq c\right] \mid \mathscr{G}_{L}\right) \mid \mathscr{G}_{l}\right) .
\end{aligned}
$$

As in (68) we can suppose that the closest ancestor $u$ for $v$ and $w$ is at height $h \geq l$.

Similar to 80 ,

$$
\begin{aligned}
& \operatorname{Cov}\left(\mathbf{E}\left(\xi_{v} \mathbf{1}\left[\xi_{v} \leq c\right] \mid \mathscr{G}_{L}\right), \mathbf{E}\left(\xi_{w} \mathbf{1}\left[\xi_{w} \leq c\right] \mid \mathscr{G}_{L}\right) \mid \mathscr{G}_{l}\right) \leq \\
& \mathbf{E}\left(\mathbf{E}\left(\xi_{v} \mathbf{1}\left[\xi_{v} \leq c\right] \mid \mathscr{G}_{L}\right) \mathbf{E}\left(\xi_{w} \mathbf{1}\left[\xi_{w} \leq c\right] \mid \mathscr{G}_{L}\right) \mid \mathscr{G}_{l}\right) .
\end{aligned}
$$


Thus,

$$
\begin{aligned}
& \mathbf{E}\left(\operatorname{Cov}\left(\mathbf{E}\left(\xi_{v} \mathbf{1}\left[\xi_{v} \leq c\right] \mid \mathscr{G}_{L}\right), \mathbf{E}\left(\xi_{w} \mathbf{1}\left[\xi_{w} \leq c\right] \mid \mathscr{G}_{L}\right) \mid \mathscr{G}_{l}\right)\right) \leq \\
& \mathbf{E}\left(\mathbf{E}\left(\xi_{v} \mathbf{1}\left[\xi_{v} \leq c\right] \mid \mathscr{G}_{L}\right) \mathbf{E}\left(\xi_{w} \mathbf{1}\left[\xi_{w} \leq c\right] \mid \mathscr{G}_{L}\right)\right) .
\end{aligned}
$$

We use calculations as in (52) to get for a vertex $v$ with $h(v)=j$,

$$
\mathbf{E}\left(\xi_{v} \mathbf{1}\left[\xi_{v} \leq c\right] \mid \mathscr{G}_{L}\right)=\frac{m n_{v}}{n(m+1)} e^{-\frac{m+1}{m} \ln _{+}\left(\frac{m n_{v}}{n c}\right)} \leq \frac{n_{v}}{n} \leq \prod_{r=1}^{j} U_{r, v} .
$$

Denote by $\left(v_{u}, w_{u}\right)$ a pair of vertices with closest ancestor $u$ as in (82). Consider one such pair $\left(v_{u}, w_{u}\right)$ and let $h=h(u), j=h(v)$ and $k=h(w)$.

Since $\mathbf{E}\left(U_{r, v}\right)=\frac{1}{2}$ and $\mathbf{E}\left(U_{h, u}\left(1-U_{h, u}\right)=\frac{1}{6}<\frac{1}{4}\right.$, where $u$ with $h(u)=h$ is the closest ancestor of $v$ and $w$, it follows that

$$
\begin{aligned}
\mathbf{E}\left(\mathbf{E}\left(\xi_{v_{u}} \mathbf{1}\left[\xi_{v_{u}} \leq c\right] \mid \mathscr{G}_{L}\right) \mathbf{E}\left(\xi_{w_{u}} \mathbf{1}\left[\xi_{w_{u}} \leq c\right] \mid \mathscr{G}_{L}\right)\right) & \leq \mathbf{E} \prod_{r=1}^{h} U_{r, u}^{2} 2^{-(j-h)-(k-h)} \\
& =\left(\frac{1}{3}\right)^{h} 2^{-(j-h)-(k-h)}
\end{aligned}
$$

Thus, by using (98)-(102) and letting $v, w: v \wedge w=u$ denote that the vertices $v, w$ have closest ancestor $u$ as in (84) and (85) we get

$$
\begin{aligned}
& \mathbf{E}\left(\operatorname{Var}\left(\sum_{l \leq h(v) \leq L} \mathbf{E}\left(\xi_{v} \mathbf{1}\left[\xi_{v} \leq c\right] \mid \mathscr{G}_{L}\right) \mid \mathscr{G}_{l}\right)\right) \\
& =\sum_{h=l}^{L} \sum_{\substack{u: h(u)=h \\
\sum_{v, w \in T_{u}: v \wedge w=u}}} \mathbf{E}\left(\mathbf{E}\left(\xi_{v} \mathbf{1}\left[\xi_{v} \leq c\right] \mid \mathscr{G}_{L}\right) \mathbf{E}\left(\xi_{w} \mathbf{1}\left[\xi_{w} \leq c\right] \mid \mathscr{G}_{L}\right)\right) \\
& =\sum_{h=l}^{L} \sum_{\substack{u: h(u)=h \\
\sum_{h \leq j \leq L}}} \sum_{\substack{v \leq k \leq L \\
h \leq h, h(v)=j, h(w)=k}} \mathbf{E}\left(\mathbf{E}\left(\xi_{v} \mathbf{1}\left[\xi_{v} \leq c\right] \mid \mathscr{G}_{L}\right) \mathbf{E}\left(\xi_{w} \mathbf{1}\left[\xi_{w} \leq c\right] \mid \mathscr{G}_{L}\right)\right) \\
& \leq \sum_{h=l}^{L}\left(\frac{1}{3}\right)^{h} 2^{h} \sum_{j=h}^{L} 2^{j-h-(j-h)} \sum_{k=h}^{L} 2^{k-h-(k-h)} \leq 3 L^{2}\left(\frac{2}{3}\right)^{l} \rightarrow 0 .
\end{aligned}
$$

We now show that

$$
\mathbf{E}\left(\operatorname{Var}\left(\frac{4 \ln ^{2} n}{n} \sum_{h(v)=L} \frac{n_{v}}{2 \ln n_{v}} \mid \mathscr{G}_{l}\right)\right) \rightarrow 0
$$



show,

Using the expansion of $\sum_{h(v)=L} \frac{n_{v}}{2 \ln n_{v}}$ in (59) and (60) we only need to

$$
\mathbf{E}\left(\operatorname{Var}\left(\sum_{v: h(v)=L} \prod_{r=1}^{L} U_{r, v} \sum_{r=1}^{L} \ln U_{r, v} \mid \mathscr{G}_{l}\right)\right) \rightarrow 0
$$

Using (103) we obtain for each $s \leq L$,

$$
\mathbf{E}\left(\operatorname{Var}\left(\sum_{v: h(v)=L} \prod_{r=1}^{L} U_{r, v} \ln U_{s, v} \mid \mathscr{G}_{l}\right)\right)=\mathcal{O}\left(L^{2}\left(\frac{2}{3}\right)^{l}\right) .
$$

Thus, applying the conditional Hölder inequality, see e.g. [9, p. 476], we get

$$
\mathbf{E}\left(\operatorname{Var}\left(\sum_{v: h(v)=L} \prod_{r=1}^{L} U_{r, v} \sum_{r=1}^{L} \ln U_{r, v} \mid \mathscr{G}_{l}\right)\right)=\mathcal{O}\left(L^{4}\left(\frac{2}{3}\right)^{l}\right)
$$

Hence,

$$
\mathbf{E}\left(\operatorname{Var}\left(\frac{4 \ln ^{2} n}{n} \sum_{h(v)=L} \frac{n_{v}}{2 \ln n_{v}} \mid \mathscr{G}_{l}\right)\right) \rightarrow 0
$$

Using the conditional Hölder inequality again gives

$$
\begin{aligned}
& \operatorname{Cov}\left(\sum_{l \leq h(v) \leq L} \mathbf{E}\left(\xi_{v} \mathbf{1}\left[\xi_{v} \leq c\right] \mid \mathscr{G}_{L}\right), \sum_{h(v)=L} \frac{n_{v}}{2 \ln n_{v}} \mid \mathscr{G}_{l}\right) \\
& \leq \max \left(\operatorname{Var}\left(\sum_{l \leq h(v) \leq L} \mathbf{E}\left(\xi_{v} \mathbf{1}\left[\xi_{v} \leq c\right] \mid \mathscr{G}_{L}\right) \mid \mathscr{G}_{l}\right), \frac{4 \ln ^{2} n}{n} \operatorname{Var}\left(\sum_{h(v)=L} \frac{n_{v}}{2 \ln n_{v}} \mid \mathscr{G}_{l}\right)\right) .
\end{aligned}
$$

Thus, (103) and (104) imply

$$
\mathbf{E}\left(\operatorname{Var}\left(\sum_{l \leq h(v) \leq L} \mathbf{E}\left(\xi_{v} \mathbf{1}\left[\xi_{v} \leq c\right] \mid \mathscr{G}_{L}\right)-\frac{4 \ln ^{2} n}{n} \sum_{h(v)=L} \frac{n_{v}}{2 \ln n_{v}} \mid \mathscr{G}_{l}\right)\right) \rightarrow 0
$$

which together with (97) proves (69). 
For (70) we proceed with the same method as for showing (68) and (69). Using (73) one gets (by similar calculations as in (64) and (65)),

$$
\begin{aligned}
& \mathbf{E}\left(\sum_{l \leq h(v) \leq L} \operatorname{Var}\left(\xi_{v} \mathbf{1}\left[\xi_{v} \leq c\right] \mid \mathscr{G}_{L}\right) \mid \mathscr{G}_{l}\right) \\
& =\sum_{i=1}^{2^{l}} \sum_{j=l+1}^{L} \frac{2^{j-l} m}{2} \mathbf{E}\left(\prod_{r=1}^{l} U_{r, v_{i}}^{2} \prod_{r=l+1}^{j} U_{r, v}^{2}\right. \\
& \left.\quad \cdot e^{-\frac{2 m+1}{m}\left(\ln m-\ln c-Y_{l, v_{i}}-Y_{j-l, v}\right) I\left\{Y_{l, v_{i}}+Y_{j-l, v} \leq \ln m-\ln c\right\}} \mid \mathscr{G}_{l}\right)+o(1) \\
& =I_{1}+I_{2}+o(1),
\end{aligned}
$$

where

$$
\begin{aligned}
I_{1}:=\sum_{i=1}^{2^{l}} \sum_{j=l+1}^{L} \frac{2^{j-l} m}{2} & \int_{0}^{\ln m-\ln c-Y_{l, v_{i}}}\left(e^{-2\left(Y_{l, v_{i}}+y_{j-l, v}\right)}\right. \\
& \left.\cdot e^{-\frac{2 m+1}{m}\left(\ln m-\ln c-Y_{l, v_{i}}-y_{j-l, v}\right)} \frac{e^{-y_{j-l, v}} y_{j-l, v}^{j-l-1}}{(j-l-1) !}\right) d y_{j-l, v}
\end{aligned}
$$

and

$I_{2}:=\sum_{i=1}^{2^{l}} \mathbf{E}\left(\sum_{j=l+1}^{L} \frac{2^{j-l} m}{2} \prod_{r=1}^{l} U_{r, v_{i}}^{2} \prod_{r=l+1}^{j} U_{r, v}^{2}\left(1-I\left\{Y_{l, v_{i}}+Y_{j-l, v} \leq \ln m-\ln c\right\}\right) \mid \mathscr{G}_{l}\right)$.

We calculate (similarly as in (66) and (67)),

$$
\begin{aligned}
I_{1} & =\sum_{i=1}^{2^{l}} \int_{0}^{\ln m-\ln c-Y_{l, v_{i}}} m e^{-Y_{l, v_{i}}} e^{-\frac{2 m+1}{m}(\ln m-\ln c)} e^{\frac{m+1}{m}\left(Y_{l, v_{i}}+y_{j-l, v}\right)} d y_{j-l, v}+o(1) \\
& =\sum_{i=1}^{2^{l}}\left|e^{-y_{l, v_{i}}} \frac{m^{2}}{m+1} e^{-\frac{2 m+1}{m}(\ln m-\ln c)} e^{\frac{m+1}{m}\left(Y_{l, v_{i}}+y_{j-l, v}\right)}\right|_{0}^{\ln m-\ln c-Y_{l, v_{i}}}+o(1) \\
& =\sum_{i=1}^{2^{l}} \prod_{r=1}^{l} U_{r, v_{i}} \frac{m^{2}}{m+1} e^{-(\ln m-\ln c)}+o(1)=\sum_{i=1}^{2^{l}} \prod_{r=1}^{l} U_{r, v_{i}} \frac{m^{2} c}{m(m+1)}+o(1) \\
& =\sum_{i=1}^{2^{l}} \prod_{r=1}^{l} U_{r, v_{i}} c+o(1)=c+o(1)
\end{aligned}
$$


and

$$
\begin{aligned}
I_{2} & =\sum_{i=1}^{2^{l}} \prod_{r=1}^{l} U_{r, v_{i}}^{2} \sum_{j=l+1}^{L} \frac{2^{j-l} m}{2 \cdot 3^{j-l}} \\
& -\sum_{i=1}^{2^{l}} \int_{0}^{\ln m-\ln c-Y_{l, v_{i}}} \sum_{j=l+1}^{L} 2^{j-l} m e^{-2\left(Y_{l, v_{i}}+y_{j-l, v}\right)} \frac{e^{-y_{j-l, v}} y_{j-l, v}^{j-l-1}}{(j-l-1) !} d y_{j-l, v}+o(1) \\
= & \sum_{i=1}^{2^{l}} \prod_{r=1}^{l} U_{r, v_{i}}^{2} m-\sum_{i=1}^{2^{l}} \int_{0}^{\ln m-\ln c-Y_{l, v_{i}}} e^{-Y_{l, v_{i}} m e^{-Y_{l, v_{i}}-y_{j-l, v}} d y_{j-l, v}+o(1)} \\
= & \sum_{i=1}^{2^{l}} \prod_{r=1}^{l} U_{r, v_{i}} c+o(1)=c+o(1) .
\end{aligned}
$$

Thus,

$$
\operatorname{Var}\left(\mathbf{E}\left(\sum_{l \leq h(v) \leq L} \operatorname{Var}\left(\xi_{v} \mathbf{1}\left[\xi_{v} \leq c\right] \mid \mathscr{G}_{L}\right) \mid \mathscr{G}_{l}\right)\right) \rightarrow 0
$$

It remains to show that

$$
\mathbf{E}\left(\operatorname{Var}\left(\sum_{l \leq h(v) \leq L} \operatorname{Var}\left(\xi_{v} \mathbf{1}\left[\xi_{v} \leq c\right] \mid \mathscr{G}_{L}\right) \mid \mathscr{G}_{l}\right)\right) \rightarrow 0 .
$$

To show this we observe that

$$
\operatorname{Var}\left(\xi_{v} \mathbf{1}\left[\xi_{v} \leq c\right] \mid \mathscr{G}_{L}\right) \leq \mathbf{E}\left(\xi_{v}^{2} \mathbf{1}\left[\xi_{v} \leq c\right] \mid \mathscr{G}_{L}\right) \leq c \mathbf{E}\left(\xi_{v} \mathbf{1}\left[\xi_{v} \leq c\right] \mid \mathscr{G}_{L}\right),
$$

and thus (115) follows from (102) by similar calculations as in (103). Hence, (115) together with (114) show (70).

We have proved Theorem 2.1 .

\section{Acknowledgement :}

I wish to gratefully acknowledge the help and support of Professor Svante Janson, who both presented me with the problem and gave invaluable guidance and supervision throughout the work. 


\section{References}

[1] B. Chauvin, M. Drmota, and J. Jabbour Hattab, The profile of binary search trees. Ann. Applied Probab. 11, 2001, 1042-1062.

[2] L. Devroye, A note on the height of binary search trees. J. Assoc. Comput. Mach. 33, 1986, 489-498.

[3] L. Devroye, Branching processes and their applications in the analysis of tree structures and tree algorithms. Probabilistic Methods for Algorithmic Discrete Mathematics, ed. Habib, et al. Springer Series on Algorithms and Combinatorics, vol. 16, Springer, Berlin, 1998, 249-314.

[4] L. Devroye, Applications of Steins method in the analysis of random binary search trees. Steins method and Applications, ed. Chen and Barbour, Institute for Mathematical Sciences Lecture Notes Series, vol. 5, World Scientific Press, Singapore, 2005, 247-297.

[5] M. Drmota, Profile and height of random binary search trees, 2003.

[6] M. Drmota, A. Iksanov, M. Moehle and U. Roesler, A limiting distribution for the number of cuts needed to isolate the root of a random recursive tree. Preprint, 2006.

[7] J. A. Fill and S. Janson, Quicksort asymptotics. J. Algorithms 44, 2002, $4-28$.

[8] W. Feller, An Introduction to Probability Theory and Its Applications. Volume II 2nd edition, Wiley, New York, 1971.

[9] A. Gut, Probability: A Graduate Course, Springer Texts in Statistics, Springer, New York, 2005.

[10] A. Iksanov, M. Moehle, A probabilistic proof of a weak limit law for the number of cuts needed to isolate the root of a random recursive tree. Preprint, 2006.

[11] S. Janson, Random records and cuttings in complete binary trees. Mathematics and Computer Science III, Algorithms, Trees, Combinatorics and Probabilities (Vienna, 2004,) Birkhäuser, Basel/Switzerland, 2004, 241-253.

[12] S. Janson, Random cuttings and records in deterministic and random trees. Random Struct. Alg. 29, 2006, 139-179. 
[13] S. Janson, T. Łuczak, A. Rucinski, Random Graphs. Wiley Interscience Series in Discrete Mathematics and Optimization, Wiley, New York, 2000 .

[14] O. Kallenberg, Foundations of Modern Probability. 2nd edition, Springer Verlag, Reading, Mass., 2002.

[15] C. Knessl, W. Szpankowski, Quicksort algorithm again revisited. Discrete Math. Theor. Comput. Sci. no. 2 (electronic) 1999, 43-64.

[16] D. E. Knuth, The Art of Computer Programming. Volume 1: Fundamental Algorithms. 3rd edition, Addison Wesley, Reading Mass., 1997.

[17] D. E. Knuth, The Art of Computer Programming. Volume 3: Sorting and Searching. Addison-Wesley, New York, 2002.

[18] A. Meir and J. W. Moon, Cutting down random trees. J. Australian Math. Soc. 11, 1970, 313-324. 